\title{
UICN
}

\section{Directives relatives à la planification et à la gestion des espèces envahissantes dans les îles}
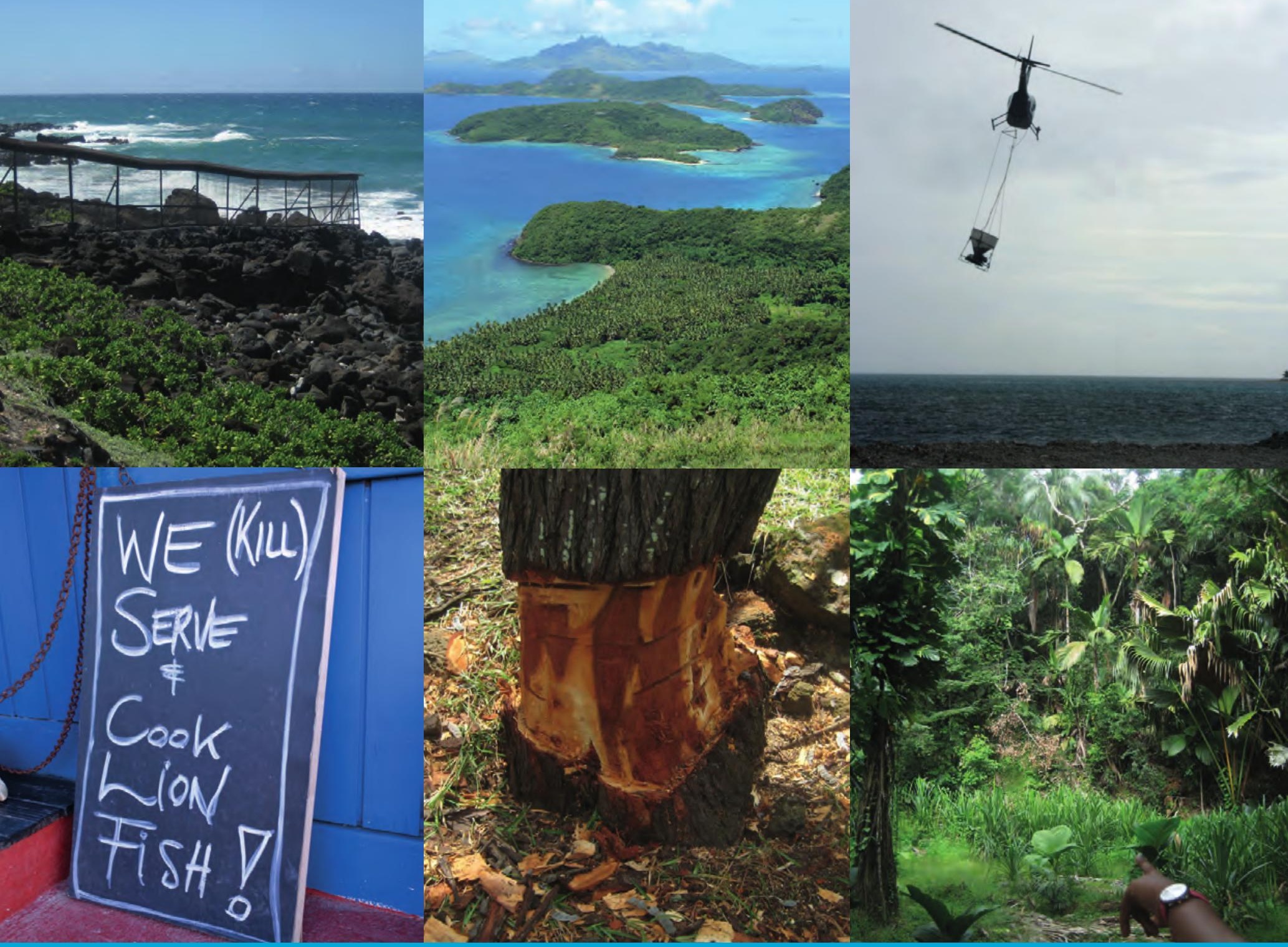

PROGRAMME MONDIAL DES ESPĖCES

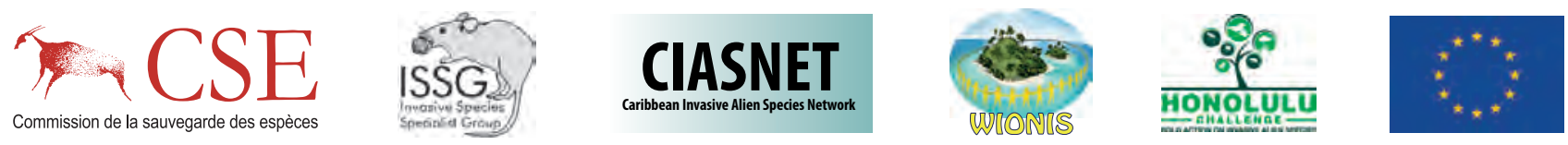





\section{Directives relatives à la planification et à la gestion des espèces envahissantes dans les îles}


La terminologie géographique employée dans cet ouvrage, de même que sa présentation, ne sont en aucune manière l'expression d'une opinion quelconque de la part de l'UICN, de la Commission européenne, ou des autres organisations concernées sur le statut juridique ou l'autorité de quelque pays, territoire ou région que ce soit, ou sur la délimitation de ses frontières.

Les opinions exprimées dans cette publication ne reflètent pas nécessairement celles de l'UICN, la Commission européenne, ou des autres organisations qui ont contribué à cet ouvrage.

L'UICN et les autres organisations concernées rejettent toute responsabilité en cas d'erreurs ou d'omissions intervenues lors de la traduction en français de ce document dont la version originale est en anglais. En cas de divergences, veuillez-vous référer à l'édition originale. Titre de l'édition originale : Guidelines for invasive species planning and management on islands (2018). Publié par : UICN Cambridge, Royaume-Uni et Gland, Suisse.

Le présent ouvrage a pu être publié grâce à un soutien financier de l'Union européenne dans le cadre du projet DCl/ENV/2009/6/8 Etablir et tester un modèle compréhensif pour prévenir et gérer l'extension des espèces envahissantes dans les écosystèmes insulaires (Projet Inva'Ziles).

Publié par :

Droits d'auteur :

Citation:

Compiler and editor:

ISBN:

DOI:

Traduction :

Photos couverture et de l'intérieur:

Mise en page :

Imprimé par :

Disponible auprès de :
UICN Cambridge, Royaume-Uni et Gland, Suisse

(C) 2018 UICN, Union internationale pour la conservation de la nature et de ses ressources La reproduction de cette publication à des fins non commerciales, notamment éducative, est permise sans autorisation écrite préalable du détenteur des droits d'auteur à condition que la source soit dûment citée.

La reproduction de cette publication à des fins commerciales, notamment en vue de la vente, est interdite sans autorisation écrite préalable du détenteur des droits d'auteur.

UICN (2018). Directives relatives à la planification et à la gestion des espèces envahissantes dans les îles. Cambridge, Royaume-Uni et Gland, Suisse: UICN. viii + 44 pp.

Alan Tye

978-2-8317-1923-8 (PDF)

978-2-8317-1924-5 (print version)

https://doi.org/10.2305/IUCN.CH.2018.15.fr

Stefan Hart de Keating <mauritiantranslator@gmail.com>, Olivier Hasinger <Olivier.HASINGER@iucn.org>

(c) Chris Buddenhagen (page 1 au centre), Sue Daly/naturepl.com (couverture en bas à gauche), Gillian Key (page 15 centre droite), Christophe Lavergne (page 15 centre gauche) et Alan Tye (les autres photos). Toutes les photographies utilisées dans cette publication restent la propriété du détenteur original des droits d'auteur. Les photographies ne doivent pas être reproduites ou utilisées dans d'autres contextes sans l'autorisation écrite du détenteur des droits d'auteur.

NaWa design www.nawadesign.com (basé sur la mise en page de la version en anglais, par NatureBureau)

BSR Imprimeurs SA sur papier mat de $115 \mathrm{~g} / \mathrm{m} 2$, avec couverture en papier semi-mat de $240 \mathrm{~g} / \mathrm{m} 2$, imprimé sur du papier obtenu à partir de fibre de bois provenant de forêts bien gérées, certifiées selon les normes du Forest Stewardship Council (FSC).

UICN (Union internationale pour la conservation de la nature)

Programme mondial des espèces

Rue Mauverney 28

1196 Gland

Suisse

Tél +41229990000

Fax +41 229990002

www.iucn.org/resources/publications 


\section{Table des matières}

À quoi servent ces directives?

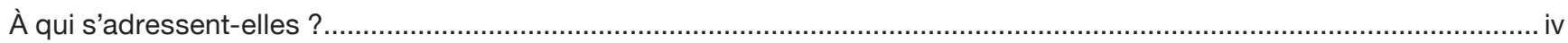

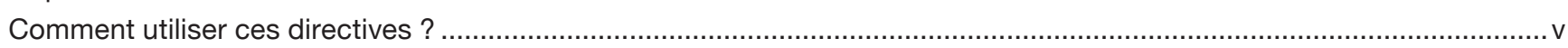

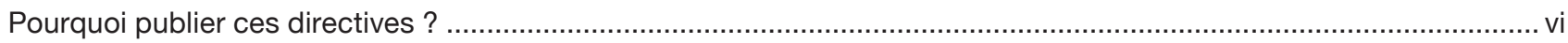

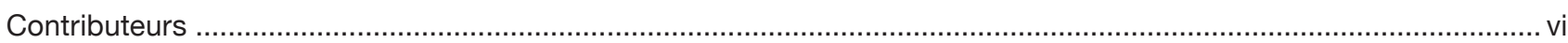

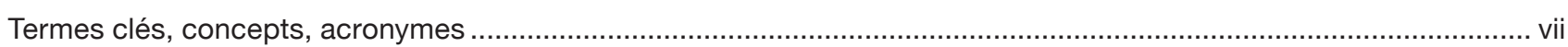

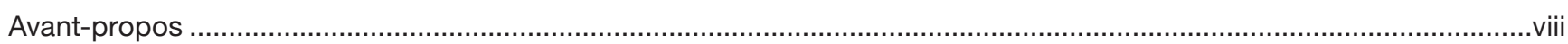

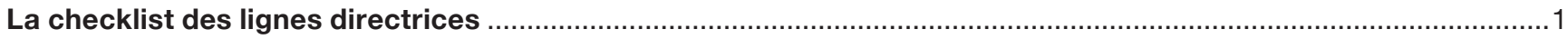

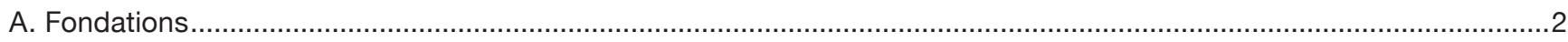

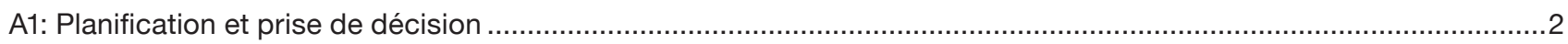

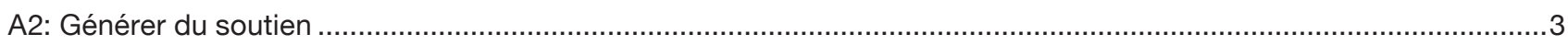

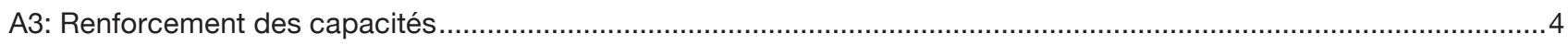

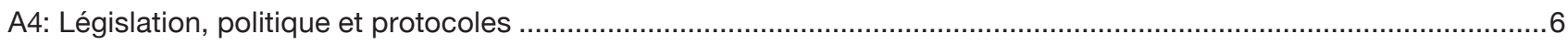

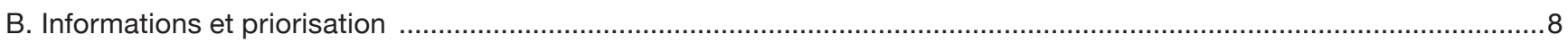

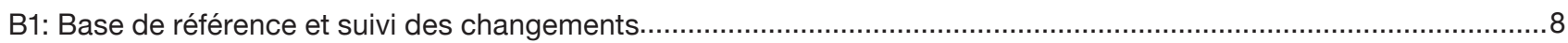

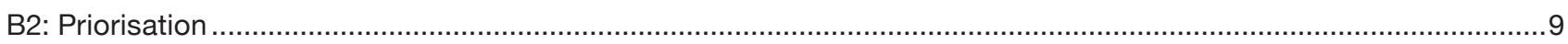

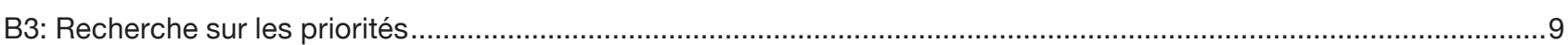

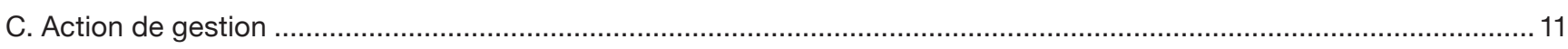

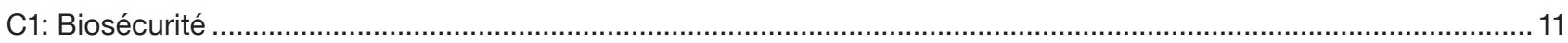

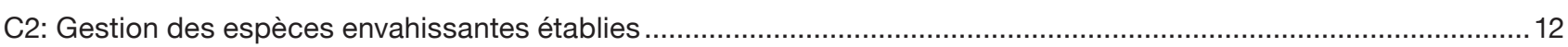

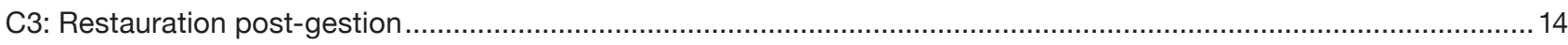

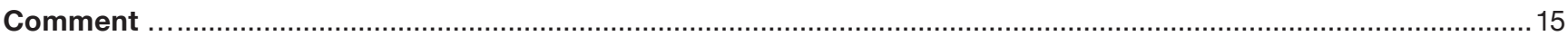

1. Comment impliquer, créer une dynamique et susciter des soutiens .................................................................16

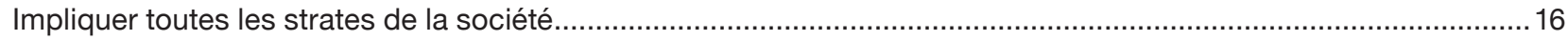

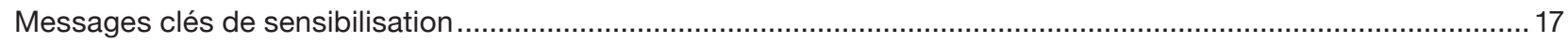

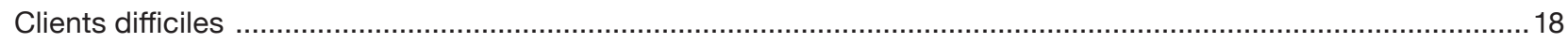

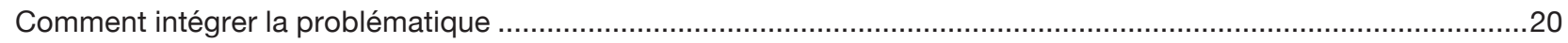

Aide mutuel - comment mettre en place des réseaux régionaux ...................................................................21

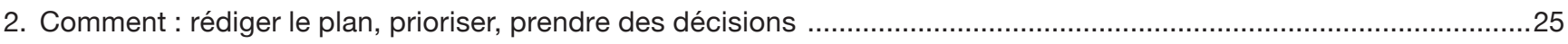

Comment constituer et faire fonctionner un Comité national sur les espèces envahissantes .................................25

Comment rédiger une stratégie et un plan d'action sur les espèces envahissantes (SPAEE) ................................25

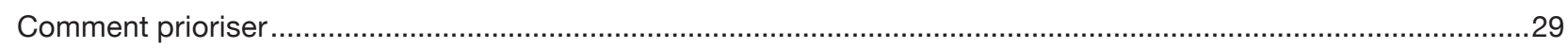

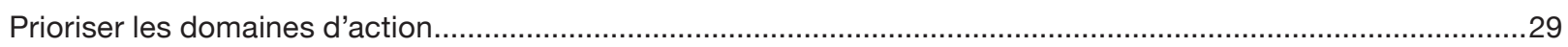

Prioriser les espèces, les voies d'introduction et les sites...............................................................................31

Comment sélectionner les buts de gestion pour une espèce ou un site ...............................................................34

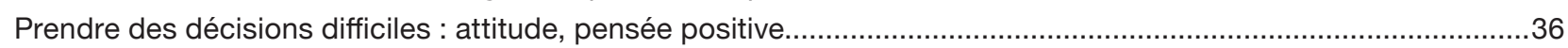

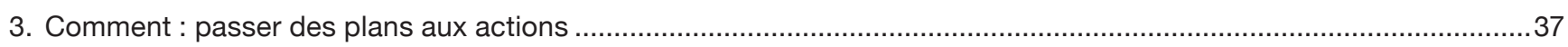

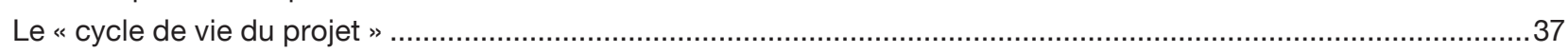

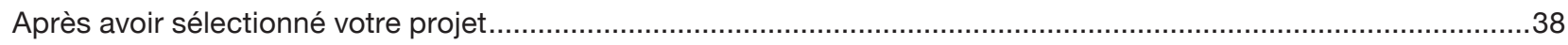

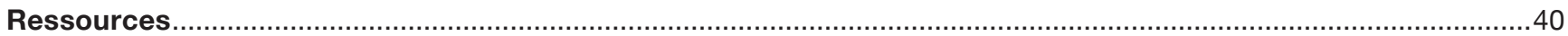




\section{À quoi servent ces directives?}

Le but de ce document est d'assister toute personne planifiant et programmant la gestion des espèces envahissantes dans les îles, avec pour objectif de réduire les impacts négatifs des espèces envahissantes sur le patrimoine naturel, les communautés et les moyens de subsistance des îles.

\section{Ces Directives visent à :}

- Fournir un cadre complet pour la gestion des espèces envahissantes dans les îles

- Aborder tous les problèmes et faciliter la hiérarchisation des priorités

- Renforcer les actions et améliorer la mise en œuvre

- Augmenter l'efficacité et la coopération, réduire la duplication des efforts

- Guider le travail des organisations internationales et régionales, y compris les donateurs

- Guider l'élaboration des programmes par pays et par île, y compris les Stratégies et Plans d'action nationaux contre les espèces envahissantes ainsi que les plans des organisations individuelles

- Guider la collecte de fonds stratégique et locale

Ces Directives traitent des plantes, des animaux, des agents pathogènes et d'autres organismes envahissants, dans les milieux marins, dulcicoles et terrestres, et de leurs impacts sur l'environnement, la biodiversité, les services écosystémiques, la santé humaine, l'économie et la société. Tous les domaines thématiques clés et leurs objectifs sont nécessaires pour la gestion globale de la menace des espèces envahissantes. Ces Directives reflètent les conventions et les normes internationales pertinentes (voir Ressources p. 40) et sont compatibles avec ces dernières. Elles ont pour but d'aider les planificateurs et les praticiens à respecter les normes acceptées et les meilleures pratiques.

\section{À qui s'adressent-elles ?}

Le présent document fournit des directives à l'intention de tous ceux qui planifient un programme sur les espèces envahissantes, n'importe où dans les îles. Il apporte un soutien aux îles pour l'élaboration de leurs plans et de leurs programmes relatifs aux espèces envahissantes ainsi que des directives aux organisations internationales et régionales pour les aider (tout au long du présent document, « régional » désigne les régions océaniques, $p$. ex., Pacifique, Caraïbes...). Les Directives sont destinées à toutes les organisations travaillant sur les espèces envahissantes dans les îles, y compris les organisations internationales et régionales, les services gouvernementaux nationaux et locaux ( $p$. ex., quarantaine, biosécurité, foresterie, agriculture, gestion de l'eau, conservation), les ONG, les gestionnaires d'aires protégées et de conservation, les scientifiques et toute autre personne qui doit trouver, planifier et prioriser des fonds et des ressources pour la gestion des espèces envahissantes et la recherche en la matière.

Aux fins des présentes Directives, les «îles » sont principalement des îles situées dans les océans, quel que soit leur taille ou leur isolement, mais les directives devraient également être utiles pour planifier les programmes liés aux espèces envahissantes partout, y compris sur les continents.

\section{Si vous travaillez pour une organisation internationale ou régionale, utilisez ces Directives pour vous aider à :}

- Identifier votre niche pour le travail sur les espèces envahissantes dans la région ou dans les régions insulaires où vous opérez.

- Identifier les besoins prioritaires des îles qui nécessitent une action de votre organisation.

- Identifier les autres organisations avec lesquelles vous pourriez ou devriez coordonner votre travail.
Si vous travaillez pour une institution ou un programme national ou local, utilisez ces Directives pour vous aider à :

- Identifier et prioriser les objectifs nécessitant une action dans votre domaine de compétence.

- Concevoir votre stratégie sur les espèces envahissantes, planifier votre programme de travail et le mettre en pratique.

- Déterminer comment coordonner votre travail avec d'autres pays et organisations, et bénéficier de leur expérience et de leur assistance.

- Justifier vos projets auprès des décideurs et des donateurs. 


\section{Comment utiliser ces directives?}

Après cette brève introduction (p. iv-viii), le document comprend deux parties principales.

La première d'entre elles (p. 1-14) est une Checklist des éléments essentiels d'un programme complet et efficace de gestion des espèces envahissantes, disposée en un ordre logique de dix Domaines thématiques, regroupés en trois Thèmes généraux (Fondations, Informations et priorisation; Action de gestion). Les dix Domaines thématiques sont à prendre en considération lors de la planification d'un programme sur les espèces envahissantes pour une île ou pour plusieurs îles.

La deuxième partie (pp. 15-38) offre des directives supplémentaires sur le processus de planification et de mise en œuvre, principalement sous la forme de «Comment » impliquer, créer une dynamique et susciter des soutiens, prendre des décisions, prioriser et passer des plans aux actions.

- Ces Directives peuvent être utilisées pour planifier et concevoir tout programme sur les espèces envahissantes, à l'échelle locale, nationale ou régionale. Elles aident à s'assurer qu'aucun aspect clé pertinent à une situation ou à un programme donné n'est oublié.

- Ces Directives sont complètes et contiennent donc de nombreux objectifs, mais tous les pays ou toutes les organisations n'ont pas besoin de tout mettre en œuvre. Votre organisation ou votre programme n'a pas besoin d'adopter tous les objectifs. Certains sont adaptés à la mise en œuvre sur le plan national ou local, tandis que d'autres nécessitent une coopération internationale ou sont plus adaptés à la mise en œuvre par des organisations régionales ou internationales. Chaque organisation peut sélectionner les objectifs qu'elle considère comme importants pour son propre programme.

- Les objectifs ne sont pas priorisés, car les priorités et les besoins diffèrent selon les îles, les pays et les territoires. Les Directives facilitent la hiérarchisation des priorités par votre pays, territoire, île ou organisation; elles ne définissent pas de priorités pour vous.

\section{Ne vous laissez pas décourager - commencez petit !}

Ce document peut sembler compliqué, mais ne vous inquiétez pas : si vous travaillez sur un plan simple avec des ressources très limitées, il peut malgré tout vous être très utile. Consultez la partie « Comment » pour y obtenir de bons conseils (en particulier, les pp. 25-28), et utilisez la Checklist pour vous donner des idées. Ces Directives sont écrites pour vous aider, non pas pour vous faire perdre votre temps. II se peut que vous pensiez que vous n'avez aucune chance de mettre en œuvre même juste quelques actions, et vous vous demandez par où commencer. Concentrez-vous sur les actions suivantes que les îles à capacité limitée peuvent adopter comme priorités principales :

- Identifiez les institutions clés et les experts disponibles sur l'île, ou les personnes ailleurs qui peuvent vous aider. Travaillez avec eux.

- Identifiez certaines des principales voies d'introduction (p. ex., les plantes de jardin, le bois non traité, les cargos infestés de rats, etc.) et travaillez à l'amélioration des contrôles de la biosécurité.

- Identifiez les succès faciles : les projets où vous pouvez vraiment réaliser l'éradication complète ou un contrôle efficace d'une espèce envahissante.

- Concentrez vos projets de gestion à long terme sur la protection des dernières parcelles d'habitat relativement non perturbées ou d'espèces indigènes fortement menacées. Choisissez un ou deux projets où vous pouvez espérer du succès.

\section{Utiliser la Checklist}

Lors de la planification, vous pouvez utiliser la partie Checklist comme point de départ et pour structurer les discussions de planification au cours des ateliers. La Checklist décrit les actions que vous pourriez inclure dans votre plan. Chaque île et chaque organisation, planifiant différents types de programmes, voudront choisir différentes actions comme priorités. En outre, différents contributeurs individuels à votre plan peuvent avoir des idées très différentes sur ce qui devrait y être inclus. La Checklist devrait vous aider à sélectionner les actions de manière plus objective, en équilibrant les suggestions faites par différentes personnes et différentes organisations. Vous pouvez utiliser les cases $\boldsymbol{V} \boldsymbol{x}$ à cocher à gauche pour indiquer que vous avez pris en compte et accepté $(\boldsymbol{V})$ ou rejeté $(\boldsymbol{X})$ chaque action, puis vous reporter à la Checklist une fois que vous avez votre projet de plan pour vous assurer qu'il comprend bien les actions acceptées $(\boldsymbol{})$.

\section{Utiliser la partie Comment}

Vous pouvez utiliser les outils d'aide à la décision et les directives dans la partie « Comment » pour vous aider à résoudre les divergences d'opinions et choisir des actions qui feront la différence et que vous pourrez accomplir avec les ressources disponibles.

\section{Planifier grâce à l'expérience}

Le processus de planification peut aller plus facilement si vous faites appel à un facilitateur expérimenté dans ce genre de planification. Ceci est fortement recommandé pour la planification à tous les niveaux, mais particulièrement pour les plans desservant divers groupes d'utilisateurs, tels que les Stratégies et Plans d'action (nationaux ou insulaires) contre les espèces envahissantes (SPAEE). Pour plus d'informations sur la planification, reportez-vous à la partie "Comment " à la page 25-38. 


\section{Pourquoi publier ces directives?}

Le taux de déplacement des plantes, des animaux et d'autres organismes au-delà de leur aire de répartition naturelle augmente fortement, en raison de l'augmentation du transport, du commerce et des voyages. Beaucoup d'espèces introduites dans de nouveaux endroits par les humains ne causent pas de problèmes dans leurs nouveaux emplacements, et beaucoup apportent des avantages considérables à l'agriculture, à l'horticulture et à la foresterie. Cependant, les " espèces envahissantes » (souvent appelées ravageurs, mauvaises herbes et maladies) sont des plantes, des animaux, des agents pathogènes et d'autres organismes qui sont délibérément ou involontairement emmenés au-delà de leur aire de répartition naturelle par des personnes et qui deviennent destructeurs de l'environnement ou des moyens de subsistance. Elles menacent la biodiversité, les ressources naturelles, la sécurité alimentaire, le développement économique, la santé humaine et les services écosystémiques tels que les ressources en eau, les cycles des nutriments et l'érosion. Les altérations de l'environnement causées par les activités humaines peuvent parfois entraîner une prolifération d'une espèce indigène avec des effets dévastateurs. Ces « espèces envahissantes indigènes » doivent aussi parfois être gérées.

Les îles sont particulièrement vulnérables aux espèces envahissantes. Les îles et leurs zones marines côtières constituent souvent des écosystèmes uniques, avec de nombreuses espèces endémiques (que l'on ne trouve que là) qui n'ont pas évolué pour faire face aux prédateurs, aux herbivores, aux mauvaises herbes hautement compétitives et aux maladies présentes sur les continents. En conséquence, les espèces introduites sont la principale cause d'extinction des espèces indigènes insulaires. Les îles sont également sujettes aux invasions, car leurs économies dépendent fortement des biens importés et des voyages (tourisme) : cela génère des taux élevés d'arrivée de nouvelles espèces et nécessite des partenariats solides pour trouver le bon équilibre entre biosécurité et moyens de subsistance durables.

Les écosystèmes insulaires sont la clé du bien-être et de la culture de 600 millions d'insulaires, soit un dixième de la population mondiale. Les espèces envahissantes dans les îles affectent l'agriculture, l'aquaculture, la pêche, la foresterie et le tourisme, réduisent la valeur des terres, endommagent les bâtiments, obstruent les voies navigables, perturbent le commerce et le transport et causent ou transmettent des maladies humaines, domestiques et végétales. La gestion des espèces envahissantes profite aux environnements insulaires, à la sécurité alimentaire, aux revenus de communauté locale, à la santé et à la société en générale\#, mais les petits états insulaires ne disposent souvent que de ressources humaines, matérielles et financières limitées pour faire face à ces menaces. Les espèces envahissantes sont un problème international, ainsi leur gestion efficace dans les îles nécessite une action coordonnée des organisations internationales et gouvernementales, des ONG, du secteur privé et des communautés locales. De bonnes ressources existent pour aider dans de nombreux aspects de la gestion des espèces envahissantes, notamment les techniques de contrôle, la biosécurité, la conception de projets, la législation, la sensibilisation et bien d'autres. Certains exemples sont répertoriés dans Ressources (pp. 40-43).

En 2010, le Programme régional océanien de l'environnement a publié les Directives relatives à la gestion des espèces envahissantes dans le Pacifique (PROE 2010), document d'orientation destiné à aider les îles du Pacifique à planifier leurs programmes sur les espèces envahissantes, lequel document a été adopté immédiatement et avec enthousiasme dans toute l'Océanie. En 2016, une consultation régionale dans l'océan Indien occidental a identifié les directives de planification comme étant une lacune dans les ressources disponibles, et une consultation mondiale subséquente à travers des listes de diffusion sur les espèces envahissantes a confirmé cette constatation et a fait ressortir une convergence de vues sur le fait que les Directives du Pacifique pourraient servir de base pour un document adapté et mis à jour en matière d'espèces envahissantes dans les îles du monde entier. D'autres consultations portant sur une ébauche basée sur les Directives du Pacifique ont eu lieu lors du Congrès mondial de la nature en 2016 et de la Island Invasives Conference de 2017, puis par des listes de diffusion. Le présent document en est le résultat. II est conçu pour être compatible avec les conventions, normes et indicateurs internationaux et régionaux pertinents, pour faciliter et coordonner leur application, et pour aider les programmes et les projets à respecter ces normes et les meilleures pratiques. Plus précisément, il est conçu de façon à faciliter le respect de la Convention sur la diversité biologique (Convention de Rio, article $8 \mathrm{~h}$ : « Chaque Partie contractante, dans la mesure du possible et selon qu'il conviendra, empêche d'introduire, contrôle ou éradique les espèces exotiques qui menacent des écosystèmes, des habitats ou des espèces »), les Objectifs d'Aichi (en particulier l'objectif 9 : «les espèces exotiques envahissantes et les voies d'introduction sont identifiées et classées en ordre de priorité, les espèces prioritaires sont contrôlées ou éradiquées et des mesures sont en place pour gérer les voies de pénétration, afin d'empêcher l'introduction et l'établissement de ces espèces ") ) et les Objectifs de développement durable, les stratégies et les plans d'action nationaux pour la biodiversité (SPANB) et les stratégies et les plans d'action nationaux contre les espèces envahissantes (voir Ressources p. 40).

\section{Contributeurs}

Ces Directives n'auraient pas pu être rédigées sans les contributions volontaires de nombreux experts en planification et en gestion des espèces envahissantes, et des utilisateurs potentiels dans les îles du monde entier, qui nous ont précisé le type de directives dont ils avaient besoin. Tous les contributeurs sont nommés ci-dessous. Les Directives du Pacifique ont également été produites avec les contributions de nombreuses personnes, dont quelques-uns ont contribuées à la version actuelle. Sans ces précieux apports sur de nombreuses années, ce document aurait été incomplet et incapable de prétendre représenter les îles du monde entier. Nous espérons que ce large éventail de contributions favorisera l'appropriation du document par toutes les îles, les ONG et les autres organisations qui cherchent à gérer les espèces envahissantes dans le monde entier. 
Le directeur général du PROE, Kosi Latu, a aimablement autorisé l'adaptation et l'utilisation des matériaux tirés des Directives relatives à la gestion des espèces envahissantes dans le Pacifique du PROE. Le présent document a été compilé et édité par Alan Tye, qui a également écrit une grande partie du texte et incorporé les contributions de 103 experts (nommés ci-dessous) issues des îles et des institutions du monde entier. Des parties importantes ont été rédigées par Souad Boudjelas (Pacifique), Alison Copeland (Bermudes), Geoffrey Howard (UICN), Jill Key (Pacifique et Territoires britanniques d'outre-mer), Ulrike Krauss (Sainte-Lucie) et Olivier Tyack (UICN). Piero Genovesi et Jill Key ont révisé une version complète du document. D'autres contributions, suggestions, révisions et encouragements ont été fournis par Ademola Ajagbe, Katy Beaver, Alex Bond, Elsa Bonnard, Olaf Booy, Rafael Borroto, Nancy Bunbury, Earl Campbell, Dario Capizzi, Juli Caujapé-Castells, Ana Costa, Franck Courchamp, Phil Cowan, Steve Cranwell, Cathleen Cybèle, Curt Daehler, Maria Cristina Duarte, Julia Dunn, Rui Bento Elias, Marco Filipovic, Julian Fitter, Frauke Fleischer-Dogley, Jason Goldberg, Ines Gómez, Viliami Hakaumotu, Sjúrður Hammer, Chad Hanson, Olivier Hasinger, Lynley Hayes, Ben Hoffmann, Nick Holmes, Stephanie Hudin, Jason Jack, Patricia Jaramillo, Marie-May Jeremie-Muzungaile, Gabe Johnson, Chris Kaiser-Bunbury, Springer Kaye, Inti Keith, John Kelly, Michael Kiehn, Cynthia Kolar, Christoph Kueffer, Marta López Darias, Janice Lord, Ian MacDonald, Gwen Maggs, Christy Martin, Kelly Martinou, John Mauremootoo, Mathilde Meheut, Tommy Melo, Jean-Yves Meyer, Joel Miles, Aileen Mill, James Millett, Nitya Mohanty, Craig Morley, David Moverley, Bradley Myer, Rachel Neville, Ray Nias, Kimberley O'Connor, Warea Orapa, Shyama Pagad, Julián Pérez, John Pinel, Bruce Potter, Parmenanda Ragen, Frida Razafinaivo, Tim Riding, Gérard Rocamora, James Russell, Susana Saavedra, Adrian Schiavini, Richard Selman, Nirmal Shah, Greg Sherley, Andy Sheppard, Junko Shimura, Didier Slachmuylders, Kevin Smith, Antonio Soares, Yohann Soubeyran, Vikash Tatayah, Sophie Thomas, Anna Traveset, Magdalena Vicens, Jeanne Wagner, Josua Wainiqolo, Katherine Walls, Andrew Walsh, Marc Woodhall, Masahito Yoshida, Glyn Young et Kristi Young. Des réunions consultatives ont été organisées par Olivier Hasinger, David Moverley, Ray Nias, Kevin Smith et Alan Tye. Conception et mise en page par Nathalie Wagner, Kevin Smith et Alan Tye.

\section{Termes clés, concepts, acronymes}

La plupart des termes, concepts et acronymes utilisés dans ce document sont définis lors de leur première apparition dans le texte. Ceux qui reviennent le plus fréquemment sont répertoriés ici pour faciliter la consultation. Certains des termes ci-dessous sont définis dans un sens restreint aux fins du présent document, dans le contexte particulier des îles. Veuillez trouver ici <https://www.cbd.int/invasive/terms.shtml> un glossaire de la terminologie des espèces envahissantes.

\section{biosécurité}

confinement

contrôle

éradication

espèce envahissante espèce indigène

\section{espèce indigène envahissante}

espèce introduite

gestion efficace

\section{lutte ou contrôle biologique}

région, régional(e) suivi

surveillance voie d'introduction
Prévenir la propagation des espèces envahissantes au-delà des frontières internationales ou intérieures, y compris entre les îles (souvent appelée «prévention »)

Maintenir une espèce envahissante dans une zone définie.

Réduire la population d'une espèce envahissante.

Élimination complète d'une espèce envahissante dans une île (ou dans une autre zone isolée).

Une espèce introduite qui devient destructrice pour l'environnement ou les intérêts humains.

Espèce qui se trouve naturellement dans une île ou dans une zone particulière, y ayant évolué ou y étant arrivée sans intervention humaine.

Une espèce indigène qui prolifère et devient destructrice à la suite des altérations de l'environnement causées par les activités humaines.

Organisme végétal, animal ou autre sorti délibérément ou involontairement de son aire de répartition naturelle. Également appelé espèce « exotique » ou «non indigène ».

Réussir sur le plan opérationnel (p. ex., en réduisant l'abondance de l'espèce à un niveau défini) et atteindre les résultats souhaités (impact réduit, rétablissement des systèmes perturbés) par rapport à la gestion des espèces envahissantes.

Contrôle d'une espèce envahissante en introduisant un ennemi naturel, tel qu'un insecte ou un champignon, qui attaque particulièrement l'espèce ciblée et n'attaque pas les espèces indigènes ou économiquement importantes.

Les îles dans un océan en particulier ou dans une partie d'un océan.

Programmes pour détecter tout changement, p. ex. dans la distribution des espèces envahissantes, le succès des projets de gestion, etc.

Suivi pour détecter de nouvelles incursions d'espèces envahissantes.

Processus ou mécanisme par lesquels une espèce est déplacée de sa zone d'origine dans une nouvelle zone.
CNEE

ONG

PILN

PIP

PROE

SPAEE

SPANB

WIONIS
Conseil national sur les espèces envahissantes

Organisation non gouvernementale

Réseau d'apprentissage sur les espèces envahissantes du Pacifique

Partenariat océanien sur les espèces envahissantes

Programme régional océanien de l'environnement

Stratégie et plan d'action sur les espèces envahissantes

Stratégie et plan d'action nationaux relatifs à la biodiversité

Réseau de l'océan Indien occidental sur les espèces envahissantes 


\section{Avant-propos}

\section{du Président de la République des Palaos}

Ici, dans le Pacifique, nous aimons penser que nous sommes des leaders mondiaux en matière d'engagement envers la gestion efficace des espèces envahissantes, et nous sommes fiers de notre engagement reposant sur une documentation solide. Depuis 2009, nos petites nations insulaires utilisent un document d'orientation élaboré par le Programme régional océanien de l'environnement (PROE) intitulé « Directives pour la gestion des espèces envahissantes dans le Pacifique » adopté par tous les pays et territoires insulaires d'Océanie. C'est donc avec joie et avec honneur que nous constatons que l'Union internationale pour la conservation de la nature (UICN) s'est inspirée de ce document d'orientation afin de modéliser le leur pour le reste du monde, avec la permission du PROE, tout en l'actualisant et en l'élargissant avec des mesures supplémentaires.

Nos gouvernements insulaires ont utilisé les Directives du Pacifique comme base pour plusieurs de nos plans nationaux et territoriaux en matière d'espèces envahissantes, lesquels sont devenus en substance plus réalistes, plus objectifs et plus axés sur les problèmes clés que nous pouvons résoudre avec nos moyens limités. Les Directives ont également grandement contribué à rehausser le profil des espèces envahissantes dans nos programmes nationaux et ceux des organisations internationales et régionales qui nous aident.

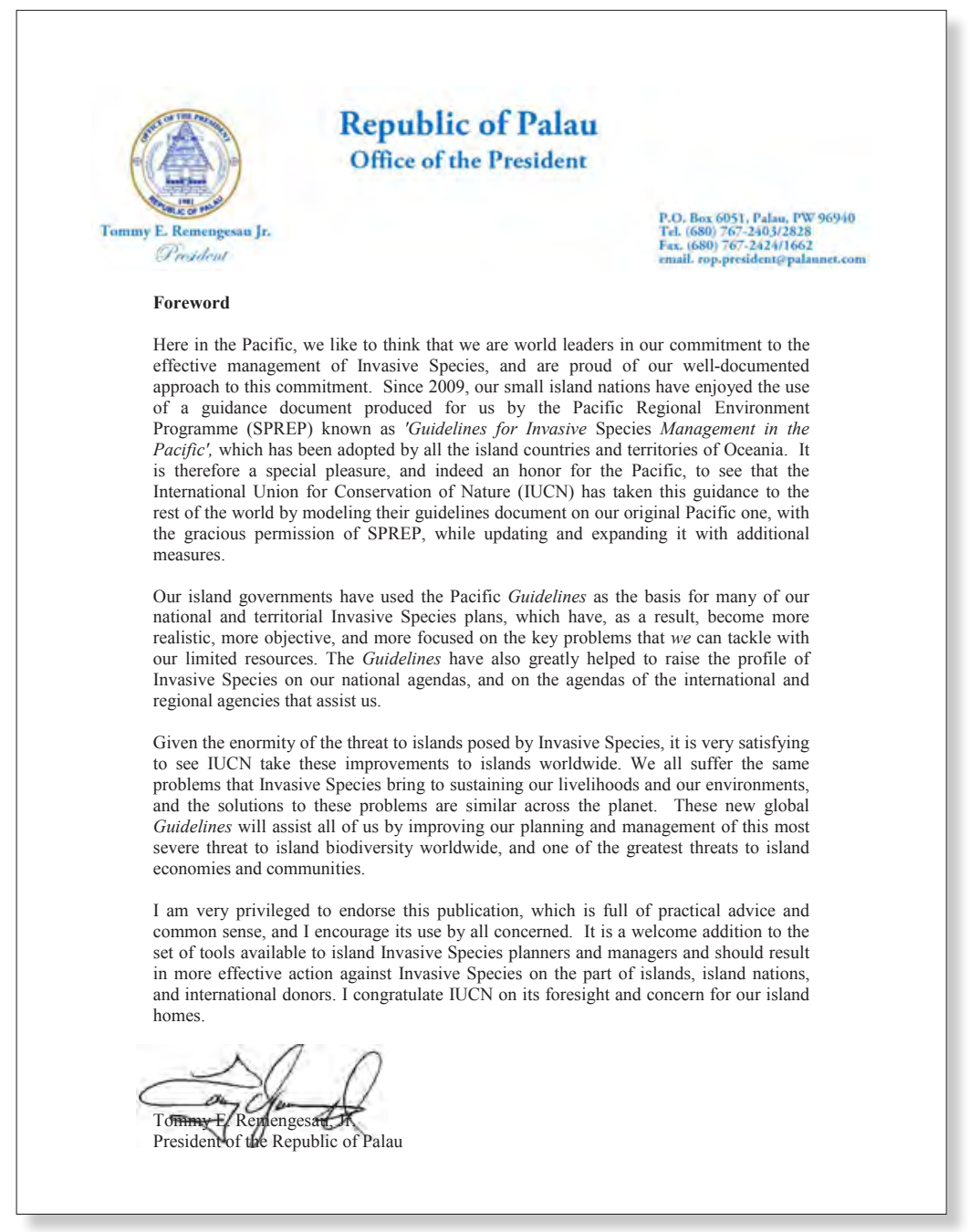

Compte tenu de l'énormité de la menace que représentent les espèces envahissantes pour les îles, il est très satisfaisant de voir l'UICN partager ces améliorations avec les îles du monde entier. Nous devons tous faire face aux mêmes effets néfastes des espèces envahissantes sur nos moyens de subsistance et nos environnements, et les solutions à ces problèmes sont similaires dans le monde entier. Ces nouvelles Directives mondiales nous aideront tous en améliorant la planification et la gestion de cette très grave menace pour la biodiversité insulaire dans le monde, et l'une des plus grandes menaces pour les économies et les communautés insulaires.

C'est pour moi un privilège de pouvoir soutenir cette publication qui est pleine de conseils pratiques et de bon sens, et j'encourage tous les intéressés à l'utiliser. Elle constitue un ajout apprécié à l'ensemble des outils disponibles pour les planificateurs et les gestionnaires des espèces envahissantes insulaires et devrait aboutir à une action plus efficace contre les espèces envahissantes de la part des îles, des nations insulaires et des donateurs internationaux. Je félicite I'UICN pour sa prévoyance et son intérêt pour nos îles.

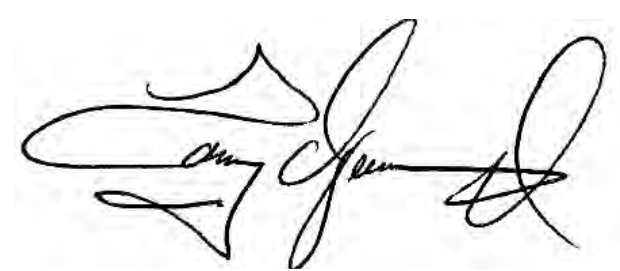

Tommy E. Remengesau Jr

Président de la République des Palaos 


\section{La Checklist des Directives $\square$}

Cette Checklist présente les éléments essentiels d'un programme complet et efficace de gestion des espèces envahissantes, organisé en un ordre logique de dix Domaines thématiques sous trois Thèmes généraux.

\section{Tous les dix Domaines thématiques doivent être pris en considération lors de} la planification d'un programme sur les espèces envahissantes pour une île ou pour plusieurs îles.

\section{A. Fondations}

A1. Planification et prise de décision: Établir des programmes stratégiques et priorisés pour la prévention et la gestion des espèces envahissantes, pour les régions océaniques, les pays et territoires insulaires, les îles individuelles et les organisations.

A2. Générer du soutien: Sensibiliser sur les impacts des espèces envahissantes sur la biodiversité, la santé humaine, les moyens de subsistance et la société, et susciter des soutiens, y compris du financement, pour des actions visant à les gérer et à les réduire.

A3. Renforcement des capacités: Développer les institutions, les compétences, l'infrastructure, le soutien technique, la gestion des informations, les réseaux et les liens nécessaires pour gérer efficacement les espèces envahissantes.

A4. Législation, politique et protocoles: Veiller à ce que les lois, les politiques, les réglementations et les procédures appropriées soient mises en place, mises en vigueur et respectées, afin de soutenir la prévention et la gestion efficaces des espèces envahissantes.

\section{B. Informations et priorisation}

B1. Base de référence et suivi des changements: Établir des informations de base sur l'état et la répartition des espèces envahissantes dans les îles, et des programmes de détection et de prévision des changements, y compris les changements d'aire de répartition et les impacts émergents.

B2. Priorisation: Établir et mettre en œuvre des systèmes efficaces d'évaluation

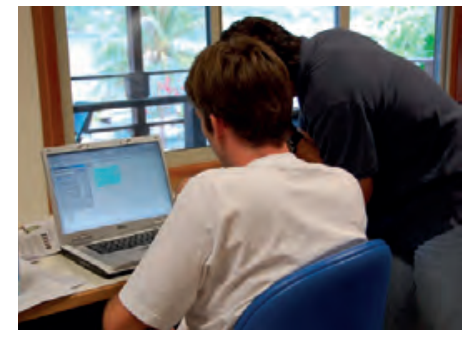
des risques et de priorisation des espèces envahissantes, des voies d'introduction et des sites à gérer.

B3. Recherche sur les priorités: Comprendre les espèces envahissantes prioritaires, y compris leur biologie et leurs impacts, et développer des techniques de gestion efficaces.

\section{Action de gestion}

C1. Biosécurité: Prévenir la propagation des espèces envahissantes au-delà des frontières internationales ou intérieures.

C2. Gestion des espèces envahissantes établies: Réduire ou éliminer les impacts des espèces envahissantes établies, par l'éradication, la lutte biologique, le confinement, l'exclusion, le contrôle physico-chimique ou l'atténuation des impacts.

C3. Restauration post-gestion: Restaurer et maintenir la biodiversité indigène, les écosystèmes, les services écosystémiques et d'autres valeurs, à la suite de la gestion des espèces envahissantes. 


\section{A. Fondations}

\section{A1: Planification et prise de décision}

Établir des programmes stratégiques et priorisés pour la prévention et la gestion des espèces envahissantes, pour les régions océaniques, les pays et territoires insulaires, les îles individuelles et les organisations.

\section{Justification}

Souvent, le contenu d'un plan dépend grandement de ceux qui y contribuent et de ceux qui s'affirment le plus, et les contributeurs individuels ont des idées très différentes au sujet de ce qui devrait y figurer. Par conséquent, les plans peuvent devenir des « listes de souhaits » trop ambitieuses ou se concentrer très sélectivement sur les intérêts d'un groupe contributeur en particulier. Pour y faire face, il faudrait des groupes et des comités multipartites formels veillant à ce que les plans tiennent compte de tous les intérêts. Des directives en matière de planification et des outils de décision peuvent être utilisés pour rendre le processus de planification aussi équilibré et objectif que possible, et aussi pour s'assurer que le plan est pratique et réaliste, avec des activités pouvant être accomplies dans un délai donné, à un coût soigneusement estimé, et en utilisant des fonds qui sont déjà disponibles ou qui peuvent être obtenus de façon réaliste en temps opportun. La gestion des espèces envahissantes est également trop rarement intégrée dans les plans d'aménagement du territoire, notamment les plans d'aires protégées, ce qui fait que les travailleurs engagés dans la lutte contre les espèces envahissantes ont besoin de mobiliser d'autres secteurs pour surmonter cela.

\section{But}

Des comités nationaux (ou insulaires) multisectoriels sur les espèces envahissantes en place, formulant, mettant en œuvre et suivant de manière efficace des plans et des programmes équilibrés, réalistes, priorisés et dotés de ressources pour leurs compétences sur un territoire. Tous les plans d'aménagement du territoire pertinents incorporent adéquatement la gestion des espèces envahissantes.

\section{Objectif}

\section{A1.1 Élaborer des} mécanismes de planification régionaux, nationaux et locaux pour guider et planifier l'action contre les espèces envahissantes dans les îles.

\section{Actions}

A1.1a Établir et maintenir des comités nationaux et locaux sur les espèces envahissantes chargés de planifier et de superviser tous les aspects de la prévention et de la gestion des espèces envahissantes dans leur domaine de compétence, avec une représentation multi-organisationelle, un soutien scientifique et des canaux de communication efficaces avec les décideurs de haut niveau, tous les secteurs pertinents et les communautés locales (voir Comment p. 25).

A1.1b Utiliser les réseaux régionaux et les mécanismes de coordination pour développer des initiatives de coopération ainsi que des priorités et des plans communs, pour la collecte de fonds et la mise en œuvre conjointes (voir A3.1, Comment pp. 21-24, Ressources p. 41).

\section{A1.2 Élaborer des stratégies} et plans d'action nationaux contre les espèces envahissantes. (voir Comment pp. 25-28)
A1.2a Élaborer des stratégies nationales ou insulaires contre les espèces envahissantes qui complèmentent les SPANB, en utilisant des méthodes de planification rationnelles et ces Directives, dans un processus consultatif incluant tous les niveaux allant du gouvernement national aux communautés locales, et avec un important soutien scientifique.

A1.2b Élaborer des plans d'action nationaux ou insulaires contre les espèces envahissantes, adaptés aux ressources et clairement définis dans le temps, pour répondre aux priorités identifiées dans les stratégies nationales, p. ex., pour des espèces et des voies d'introduction particulières, pour des sites et des écosystèmes vulnérables, etc.

A1.2c Incorporer des principes économiques dans les stratégies et les plans d'action sur les espèces envahissantes, y compris l'investissement public pour la gestion des espèces envahissantes qui engendre des bénéfices publics, et appliquer le principe de l'utilisateur-payeur lorsque les bénéfices ou les coûts sont reliés à certains secteurs en particulier.

A1.2d Profiter des occasions favorables pour intégrer la gestion des espèces envahissantes dans les plans d'autres secteurs, les plans impliquant l'aménagement du territoire, les plans pour les changements futurs (tels que le changement climatique) et tout particulièrement les plans de gestion des aires protégées ou des espèces menacées (voir Comment pp. 20-21).
A1.3 Adopter, développer et utiliser des outils et des aides à la prise de décision objective.
A1.3a Utiliser ces Directives pour guider la compilation de votre stratégie ou de votre plan de lutte contre les espèces envahissantes et, si possible, faire appel à un facilitateur expérimenté dans la planification de la gestion des espèces envahissantes (voir Comment, pp. 25-28).

A1.3b Examiner les plans existants et les nouveaux projets par rapport à la Checklist de ces Directives afin d'identifier les domaines importants négligés à incorporer dans le plan (voir Comment, pp. 29-30). 
A1.3c Utiliser les résultats de la modélisation prédictive (objectif B1.3 ci-dessous), l'évaluation des risques, l'analyse des voies d'introduction et les outils similaires (objectif B2 et la partie "Comment » sur l'évaluation des risques) pour décider des buts stratégiques et des actions prioritaires, ainsi que des espèces, des voies et des sites prioritaires (voir Comment, pp. 31-33).

A1.3d Adopter une approche hiérarchique pour sélectionner les buts de gestion (voir Comment, pp. 34-35).

A1.3e Assurez-vous que les principales priorités sont entièrement financées avant d'allouer des ressources à une priorité moindre (n'éparpillez pas les ressources).

\section{A2: Générer du soutien}

Sensibiliser sur les impacts des espèces envahissantes sur la biodiversité, la santé humaine, les moyens de subsistance et la société, et susciter des soutiens, y compris du financement, pour des actions visant à les gérer et à les réduire.

\section{Justification}

Les décideurs, le secteur privé et le grand public ont souvent une compréhension limitée des menaces que représentent les espèces envahissantes pour l'environnement, l'économie, la santé humaine et les valeurs culturelles. La gestion des espèces envahissantes est en concurrence pour le financement avec de nombreux autres intérêts. Le manque de sensibilisation, de soutien et de financement est la principale contrainte limitant la gestion des espèces envahissantes dans les îles. Un engagement actif du public et un investissement financier adéquat à long terme sont essentiels à la réussite de la gestion des espèces envahissantes. Un soutien accru et une responsabilité partagée pour la gestion des espèces envahissantes sont nécessaires, y compris la promotion des efforts individuels et la conformité volontaire.

But

Toutes les couches de la société, y compris les organismes de financement, les décideurs, les groupes d'intérêt économique et autres (gouvernement central, finances, agriculture, foresterie, horticulture, pêche, aquaculture, tourisme, transport maritime, santé publique, militaire, quarantaine) ainsi que le grand public comprennent les risques et les impacts des espèces envahissantes et les avantages de la gestion des espèces envahissantes pour la nature, l'économie et la santé humaine, et ils soutiennent tous activement la gestion des espèces envahissantes. Des ressources suffisantes sont disponibles pour permettre de répondre aux priorités nationales et régionales en matière d'espèces envahissantes.

\section{Objectif}

A2.1: Élaborer des programmes et des matériels destinés à sensibiliser les principaux groupes cibles régionaux, nationaux, sectoriels et communautaires (voir Comment pp. 16-21).

\section{$\checkmark$ Actions}

A2.1a Identifier les publics prioritaires pour les programmes de sensibilisation, y compris les décideurs, les communautés locales et les jeunes, en particulier les groupes impliqués dans la mise en œuvre des mesures de gestion des espèces envahissantes ou qui peuvent influencer leur succès.

A2.1b Rassembler les connaissances existantes par rapport aux niveaux actuels de sensibilisation et aux attitudes vis-à-vis des espèces envahissantes et des méthodes de gestion, et réaliser des études supplémentaires si nécessaire.

A2.1c Identifier et développer des messages prioritaires et des approches simples et convaincantes pour accroître la sensibilisation et le soutien des différents groupes d'intérêt sectoriels et publics, y compris les groupes de protection du bien-être des animaux.

A2.1d Offrir de la formation pour développer des campagnes médiatiques et aussi pour répondre aux médias, pour les travailleurs engagés contre les espèces envahissantes.

A2.1e Susciter la fierté des espèces indigènes et des écosystèmes, et la conscience des dommages causés par les espèces envahissantes.

A2.1f Diffuser des messages par l'intermédiaire de partenaires ayant une capacité de sensibilisation ou des voix influentes.

A2.1g Intégrer les problèmes liés aux espèces envahissantes dans les programmes de sensibilisation du public y compris sur les problèmes connexes tels que la conservation et le développement.

A2.1h Développer et exécuter des campagnes pour les messages clés ; profiter des activités et des articles qui attirent l'attention du public pour susciter l'intérêt et le soutien.

A2.1i Intégrer les espèces envahissantes dans le secteur de l'éducation formelle (primaire, secondaire, tertiaire et éducation des adultes) à travers le développement des programmes scolaires et collégiaux. 
A2.2: Assurer un soutien national, régional et international (y compris financier) en associant les problèmes liés aux espèces envahissantes à la planification de haut niveau pour tous les secteurs concernés et aux décideurs nationaux et internationaux.
A2.2a Développer des arguments et des mécanismes pour intégrer la gestion des espèces envahissantes dans les processus décisionnels nationaux, régionaux et internationaux ainsi que les plans sectoriels pour le commerce, le transport, le développement économique, l'agriculture, la pêche, l'aménagement du territoire, la gestion des aires protégées et le changement climatique.

A2.2b Utiliser ces arguments pour veiller à ce que les programmes de développement internationaux et nationaux ne favorisent pas l'introduction ni l'utilisation d'espèces envahissantes.

A2.2c Démontrer les coûts économiques potentiels liés aux espèces envahissantes connues qui ne sont pas encore présentes dans vos îles, mais avec un risque élevé d'introduction, et la nécessité de financer de manière adéquate des plans efficaces de biosécurité et de réaction rapide.

A2.2d Démontrer les coûts économiques liés aux problèmes existants causés par les espèces envahissantes dans vos îles et les avantages économiques du financement pour les gérer.

A2.2e Faire connaître les réussites en matière de prévention et de gestion des espèces envahissantes, y compris les analyses coûts-bénéfices.

A2.2f Fournir des renseignements sur les espèces envahissantes, des ateliers de formation et des ressources aux décideurs politiques.

A2.2g Assurer l'inclusion des problèmes liés aux espèces envahissantes dans les réunions régionales et nationales de haut niveau.

A2.2h Élaborer des mécanismes pour intégrer la gestion des urgences en cas de nouvelles incursions d'espèces envahissantes dans la planification et le financement de la gestion des catastrophes au niveau national et régional.
A2.3: Identifier et élaborer des mécanismes de financement externes pour le soutien de la gestion des espèces envahissantes dans les îles.
A2.3a Élaborer et publier des listes et des liens vers des sources de financement potentielles pour la gestion des espèces envahissantes et le développement des capacités.

A2.3b Utiliser ces Directives et plans d'action nationaux pour identifier les actions prioritaires nationales et régionales partagées, pour justifier les demandes de financement aux donateurs externes, en particulier pour le financement stratégique à long terme.
A2.4: Garantir le soutien des communautés locales et du secteur privé (voir Comment pp. 16-21) par rapport aux problèmes liés aux espèces envahissantes.
A2.4a Assurer l'engagement local à long terme et la durabilité du programme en promouvant la pleine participation des communautés et des conseils locaux, et du secteur privé dans tous les aspects de la gestion des espèces envahissantes, y compris la collecte d'informations et l'observation des espèces envahissantes (science citoyenne), la sensibilisation, l'identification des priorités, la prévention de l'introduction et la propagation des espèces envahissantes, l'élaboration, la gestion, la mise en œuvre et l'évaluation des projets. Fournir une rétroaction constructive et informative aux communautés concernées.

A2.4b Encourager et soutenir les priorités et les projets identifiés par les initiatives communautaires ou avec la pleine participation de la communauté.

A2.4c Développer des partenariats pour le parrainage du secteur privé.

\section{A3: Renforcement des capacités}

Développer les institutions, les compétences, l'infrastructure, le soutien technique, la gestion des informations, les réseaux et les liens nécessaires pour gérer efficacement les espèces envahissantes.

\section{Justification}

La capacité à gérer les espèces envahissantes dans les îles dépend des institutions nationales et régionales compétentes, et d'un accès facile à des infrastructures, à des équipements, à des compétences adéquates et à des informations exactes et à jour. Quand on voit l'ampleur du problème, on comprend que les îles souffrent d'un manque d'informations de base, de personnel qualifié, de planification stratégique, d'infrastructures, d'équipements et d'expertise technique et taxonomique pour la gestion des espèces envahissantes. Beaucoup d'informations sur la biologie ainsi que sur les impacts et la gestion des espèces envahissantes figurent dans des rapports locaux non publiés ou même non écrits. Les travailleurs insulaires engagés dans la lutte contre les espèces envahissantes sont souvent isolés des collègues d'autres organisations, îles et pays, et n'ont pas accès aux informations et aux compétences nécessaires pour planifier et atteindre les objectifs de gestion. La formation répétée, les services d'information, le réseautage et le partage des compétences sont donc d'une importance primordiale pour la gestion efficace des espèces envahissantes dans les îles. 
Les principales organisations collaborent et disposent d'un personnel suffisant et correctement formé. Les communautés ont la capacité de participer pleinement à la gestion des espèces envahissantes. De bonnes infrastructures et de bons équipements appropriés à la biosécurité et à la gestion sont disponibles, et des services d'identification compétents sont efficacement associés à ceux qui en ont besoin. Les informations sur la biologie, les impacts et la gestion des espèces envahissantes sont facilement accessibles à tous, établissant ainsi une base scientifique solide pour la prise de décision, l'allocation des ressources, la prédiction des impacts des espèces introduites et leur gestion. Tout ceci permet une gestion efficace et efficiente des espèces envahissantes.

\section{Objectif}

\section{A3.1 Etablir et renforcer des mécanismes qui facilitent la communication, le partage d'informations et la coopération entre les travailleurs engagés dans la lutte contre les espèces envahissantes, y compris les réseaux d'expertise, d'information, d'ateliers et d'échanges entre les îles, les institutions et les autres sources de soutien technique et scientifique.}

\section{$\checkmark$ Actions}

A3.1a Soutenir, renforcer, faire connaître et rejoindre les réseaux, les échanges et les mécanismes de partage des compétences mondiaux et régionaux sur les espèces envahissantes, y compris les listes de diffusion d'emails, et les utiliser pour partager des listes d'espèces, des expériences de gestion et d'autres informations, et pour faire des demandes d'assistance (voir Ressources p. 41).

A3.1b Coopérer pour établir, maintenir, renforcer et promouvoir des initiatives régionales de coordination et de coopération pour la gestion des espèces envahissantes, telles que le Partenariat océanien sur les espèces envahissantes (PIP), les conseils régionaux sur les espèces envahissantes de Mélanésie et de Micronésie et l'initiative du Comité français de I'UICN sur les espèces envahissantes dans les territoires d'outre-mer français.

A3.1c Utiliser les mécanismes de coopération régionale pour élaborer des priorités régionales partagées et des plans d'action concertés, et coopérer pour les suivre et les évaluer.

A3.1d Promouvoir l'échange intersectoriel en matière d'expériences et de compréhension en impliquant le plus possible les scientifiques, les gestionnaires de l'environnement et les citoyens locaux dans les projets sur les espèces envahissantes.

A3.1e Coopérer pour établir et maintenir des mécanismes mondiaux pour la coordination et la collaboration entre les organisations et les réseaux régionaux.
A3.2: Mettre en place, maintenir et faire connaître les sources régionales et nationales compétentes de soutien taxonomique, scientifique et technique pour la gestion des espèces envahissantes dans les îles de toutes les régions océaniques.
A3.2a Coopérer pour examiner et renforcer la capacité de gestion des espèces envahissantes dans les organisations régionales pertinentes (certaines sont citées dans Ressources p. 41).

A3.2b Développer et renforcer les liens avec les sources de conseils et d'assistances techniques, y compris les registres régionaux des organisations chargées de la gestion des espèces envahissantes, les experts et leur expertise, dans les régions océaniques, entre ces dernières et ailleurs.

A3.2c Coopérer pour établir et maintenir des centres de ressources régionaux pour les services essentiels en matière d'espèces envahissantes (tels que la lutte biologique, l'analyse des risques, la gestion des informations...).

A3.2d Établir et renforcer les liens avec les institutions taxonomiques, les experts, les réseaux et les services d'identification en ligne ainsi que les collections de référence d'espèces nationales et régionales (herbiers, etc.), dans les régions océaniques, entre ces dernières et ailleurs.
A3.3: Renforcer et maintenir des institutions nationales et locales compétentes avec leur personnel pour coordonner, gérer et mettre en œuvre des programmes sur les espèces envahissantes et promouvoir la pleine participation des communautés locales et des citoyens à la gestion des espèces envahissantes.
A3.3a Améliorer la coopération entre les institutions nationales et locales responsables des différents aspects de la prévention et de la gestion des espèces envahissantes, notamment par le biais des comités sur les espèces envahissantes avec l'aval du gouvernement (voir A1.1a).

A3.3b Examiner régulièrement les capacités nationales en matière de personnel à tous les niveaux et renforcer les domaines faibles, en veillant à ce que les domaines de compétences clés, les responsabilités et les ressources soient représentés ou mis à la disposition des principales organisations chargées de la gestion des espèces envahissantes. Les domaines de compétences clés correspondent grosso modo aux thèmes de ces Directives et comprennent : la politique et la planification, la facilitation de la participation communautaire, la communication avec les décideurs, la collecte de fonds, les domaines juridique, scientifique et technique (prévention et gestion), le suivi et l'évaluation. Voir A2.2 pour augmenter le soutien au renforcement des capacités. 
A3.4: Promouvoir et développer des programmes de formation couvrant tous les aspects du processus de gestion des espèces envahissantes, depuis la planification, la politique et la collecte de fonds jusqu'à la gestion pratique, la gestion des données et la diffusion des leçons apprises.
A3.4a Examiner les programmes de formation récents et actuels et identifier les lacunes importantes ainsi que les groupes cibles.

A3.4b Concevoir et mettre en œuvre des plans conjoints de formation sur les espèces envahissantes, incorporant une formation répétée à des intervalles correspondant aux taux de renouvellement du personnel, des mécanismes de renforcement des capacités après formation et une formation intersectorielle (p. ex., le personnel de biosécurité avec les douanes, le personnel du secteur agricole avec les responsables de la conservation, etc.).

A3.4c Utiliser un éventail de méthodes et de contenus de formation appropriés pour différents groupes cibles, y compris des cours formels, des échanges, la participation à des projets de démonstration, etc., et la formation des communautés locales impliquées dans le suivi et la gestion des espèces envahissantes.
A3.5: Développer ou améliorer les infrastructures régionales et nationales pour la gestion des espèces envahissantes.
A3.5a Examiner les infrastructures régionales et nationales existantes en matière de gestion des espèces envahissantes et élaborer des plans de développement à long terme.

A3.5b Établir ou renforcer des installations régionales et nationales essentielles telles que des stations d'inspection et de quarantaine et des centres de réaction rapide.

A3.5c Coopérer pour développer des institutions régionales et internationales qui fournissent des installations et des ressources indisponibles dans certains pays, comme des installations de contrôle biologique ou de réaction rapide, des services d'hélicoptères, etc., et établir des liens solides avec les utilisateurs.
A3.6: Développer, mettre à jour, faire connaître, rendre accessible et utiliser les ressources aux niveaux mondial, régional et local sur Internet et d'autres sources d'information sur les espèces envahissantes et leur gestion.
A3.6a Établir et tenir à jour des bases de données nationales et régionales sur les projets relatifs aux espèces envahissantes, y compris leurs informations techniques, leurs objectifs, leurs résultats, leurs aboutissements et les enseignements tirés.

A3.6b Coopérer pour développer, maintenir et promouvoir des ressources et des services d'information mondiaux et régionaux sur les espèces envahissantes et les meilleures pratiques (techniques de gestion, informations sur les pesticides, agents de lutte biologique et autres techniques, protocoles de suivi et de gestion des données), comme des référentiels d'informations utiles pour les îles (quelques bons exemples sont répertoriés dans Ressources pp. 41-42).

A3.6c Encourager et saisir dans les bases de données mondiales et régionales les informations provenant des îles.

A3.6d Développer des sources d'informations nationales et locales sur les espèces envahissantes, compiler et diffuser les connaissances locales et les informations de gestion, sensibiliser le public et les décideurs politiques et susciter leur soutien.

\section{A4: Législation, politique et protocoles}

Veiller à ce que les lois, les politiques, les réglementations et les procédures appropriées soient mises en place, mises en vigueur et respectées, afin de soutenir la prévention et la gestion efficaces des espèces envahissantes.

\section{Justification}

Les espèces envahissantes ont des impacts économiques, environnementaux et sociétaux, ainsi leur gestion peut impliquer une grande variété d'organisations travaillant sur différentes parties de la législation et des procédures. Les responsabilités et les lois sont souvent peu claires, inadéquates ou incompatibles. Lorsque des lois et des réglementations existent, la conformité et l'application sont souvent inappropriées. Une législation efficace et cohérente, des protocoles convenus, une conformité générale et une application efficace sont essentiels pour une action coordonnée et efficace.

\section{But}

Les îles collaborent pour mettre en place et en vigueur des cadres législatifs cohérents, alignés sur les instruments régionaux et internationaux. Les protocoles sont mutuellement convenus et suffisamment similaires pour que tous ceux qui participent à la gestion des espèces envahissantes parviennent à une coopération efficace. La législation et la réglementation sont largement comprises, généralement respectées, appliquées et soutenues par le pouvoir judiciaire. 
A4.1: Développer, promouvoir et adopter un cadre politique, juridique et d'application efficace pour la gestion des espèces envahissantes dans les îles.
A4.1a Établir, diffuser et adopter des directives législatives et stratégiques couvrant toutes les activités qui influent sur la gestion des espèces envahissantes: biosécurité, commerce, transport, construction, activités militaires, intervention en cas de catastrophe, aide au développement, recherche, agriculture, aquaculture, foresterie, horticulture, possession d'animaux de compagnie, tourisme, surveillance, analyse des risques, lutte biologique, éradication, déclaration des organismes nuisibles, etc., en utilisant les meilleurs principes et les normes internationales (voir Ressources p. 42).

A4.1b Établir des partenariats avec les facultés de droit et les services juridiques pour veiller à ce que les espèces envahissantes soient adéquatement incluses dans la législation environnementale et autres cadres légaux.

A4.1c Examiner la législation en matière d'environnement, de pêche, d'agriculture, d'aquaculture, de foresterie, d'horticulture et de biosécurité ainsi que toute autre loi pertinente dans chaque juridiction insulaire afin de déterminer si elles sont adéquates pour protéger la biodiversité, l'économie et la santé contre les espèces envahissantes et identifier les lacunes, incohérences et conflits.

A4.1d Concevoir une approche commune entre les institutions nationales et régionales quant à la politique, la législation et la gestion à suivre en matière d'espèces envahissantes.

A4.1e Veiller à ce que le cadre juridique couvre de manière exhaustive le contrôle des frontières, la réaction rapide aux nouvelles incursions, l'interdiction de déplacer et de posséder des espèces envahissantes, les exigences en matière de contrôle des espèces envahissantes et l'accès aux terres privées et publiques à gérer.

A4.1f Établir un cadre juridique qui permet et promeut la prévention et la gestion rigoureuses des espèces envahissantes dans les îles administrées par les pays continentaux, et qui régit et contrôle explicitement les déplacements entre les îles des pays insulaires.

A4.1g Assurer la pleine participation de toutes les parties prenantes, y compris le personnel technique et les communautés locales, à l'élaboration et à la mise en œuvre de la législation.

A4.1h Incorporer les principes économiques dans la législation nationale pour lutter contre les espèces envahissantes, y compris les taxes appropriées, les principes du pollueur-payeur et de l'utilisateur-payeur, et l'investissement public lorsque la gestion des espèces envahissantes engendre des bénéfices publics.

A4.1i Élaborer des mécanismes visant à améliorer le respect et l'application de la législation sur les espèces envahissantes, investir dans ces mécanismes et poursuivre efficacement les infractions.
A4.2: Élaborer et promouvoir des réglementations et des protocoles normalisés efficaces pour la gestion des espèces envahissantes dans les îles, découlant de politiques et de lois cohérentes, conformes aux exigences internationales applicables, et fondés sur des informations scientifiques et des principes de gestion solides.
A4.2a Développer des modèles de protocoles et des procédures techniques pour les îles, en établissant les meilleures pratiques pour les processus, les procédures et les objectifs de gestion communément utilisés (se référer aux Domaines thématiques C1-C3 ci-dessous).

A4.2b Réviser périodiquement les politiques et les procédures nationales et régionales sur la biosécurité, l'évaluation des risques, le commerce, les déplacements, la détention, la libération dans l'environnement, l'établissement et la gestion des espèces envahissantes, afin de se conformer aux normes internationales et aux meilleures pratiques et d'harmoniser celles-ci entre les régions océaniques.
A4.3: Assurer la pleine participation des états insulaires à l'élaboration de normes, de conventions et de programmes internationaux relatifs aux espèces envahissantes, $y$ compris sur leur circulation dans le commerce, afin de veiller à ce qu'ils reflètent les besoins des îles.
A4.3a Promouvoir et élargir les adhésions et les contributions aux conventions, comités et groupes de travail internationaux par les pays et organisations insulaires.

A4.3b Veiller à ce que les contributions nationales et régionales à l'élaboration des politiques internationales soient cohérentes et fondées sur une consultation complète entre tous les secteurs concernés. 


\section{B. Informations et priorisation}

\section{B1: Base de référence et suivi des changements 1 Établir des informations de base sur l'état et la répartition des espèces envahissantes dans les îles, et des programmes de détection et de prévision des changements, y compris les changements d'aire de répartition et les impacts émergents.}

\section{Justification}

Le manque d'information sur l'état des espèces envahissantes et potentiellement envahissantes dans les îles, y compris leurs eaux côtières et leurs récifs, entrave leur gestion. Des informations sur la distribution, l'abondance, les mécanismes de dispersion et les impacts des espèces envahissantes, ainsi que des informations sur l'abondance et la répartition de la biodiversité indigène sont essentielles pour prioriser les espèces et les sites à gérer, pour planifier des projets de gestion efficaces, pour évaluer leur succès, et comme base de référence pour le suivi des déplacements et de l'évolution des impacts des espèces envahissantes. Le suivi est également essentiel pour détecter ou prédire les tendances et les menaces émergentes telles que les effets du changement climatique sur les espèces envahissantes.

But

La connaissance de l'état actuel des espèces envahissantes et potentiellement envahissantes ainsi que de la biodiversité indigène dans les îles, y compris leurs eaux côtières et leurs récifs, est adéquate et facilement accessible, facilitant l'établissement de priorités, la planification, la gestion efficace et le suivi des changements. Des systèmes efficaces sont en place pour suivre le déplacement d'espèces introduites dans les pays et les îles et entre ces derniers, pour détecter les tendances, prévoir les changements et évaluer les résultats de la gestion.

\section{Objectif}

B1.1: Base de référence.

Générer, mettre à jour et à disposition des checklists et des informations de base sur les espèces terrestres et aquatiques introduites et indigènes, sur les voies d'introduction et sur les sites sensibles pour l'ensemble des îles et des états insulaires.

\section{Actions}

B1.1a Compiler des checklists, des bases de données SIG et d'autres ressources d'information sur les espèces indigènes et introduites, les sites à risque (c.-à- $d$., les sites fortement exposés aux espèces envahissantes telles que les ports, les jardins, les bords de routes et chemins de fer) et les sites sensibles (p. ex., les zones protégées et les systèmes aquatiques).

B1.1b Identifier et documenter les voies et les vecteurs d'introduction d'espèces envahissantes et potentiellement envahissantes (voir Comment pp. 32-33 et Ressources p. 42)

B1.1c Revoir périodiquement les inventaires d'espèces et de sites, identifier les lacunes (écosystèmes, îles ou groupes taxonomiques) et planifier les études de terrain prioritaires.

B1.1d Effectuer les études de terrain prioritaires et mettre à jour les ressources d'information.

B1.1e Publier et partager des listes d'espèces et d'autres informations localement et en collaboration avec d'autres îles, y compris sur internet et dans les bases de données répertoriées ci-dessous dans la section Ressources (pp. 41-42).

\section{B1.2: Suivi de la propagation} local ${ }^{2}$. Mettre en œuvre des techniques normalisées efficaces pour suivre la propagation des espèces envahissantes à l'intérieur des îles.
B1.2a Promouvoir, améliorer et adopter les meilleures pratiques pour le suivi de la propagation des espèces envahissantes à l'intérieur des îles, des zones naturelles sensibles et d'autres sites à haut risque.

B1.2b Élaborer et mettre en œuvre des plans de suivi pour les espèces et les sites identifiés comme prioritaires (par les méthodes de priorisation décrites dans l'objectif B2 ci-dessous) sur chaque île.
B1.3: Prédiction. Modéliser les tendances futures des invasions, y compris les effets des changements environnementaux et sociétaux majeurs tels que le changement climatique, la perte de forêts et l'évolution de la répartition des richesses.
B1.3a Engager et utiliser des experts mondiaux et régionaux et coopérer avec eux pour modéliser les futures introductions et invasions dans les îles (« tour d'horizon ») afin d'identifier les espèces potentiellement envahissantes à haut risque et leurs voies d'introduction, sur la base de la présence d'espèces envahissantes dans les îles voisines ou dans les principaux pays partenaires commerciaux, et en fonction des tendances économiques, sociétales ou environnementales réelles ou probables.

B1.3b Utiliser les résultats de la modélisation prédictive pour sélectionner les buts généraux de la planification stratégique (voir A1.3).

1 Le suivi et l'évaluation des mesures de gestion sont couverts par les objectifs C2.2 et C3.1 ci-dessous.

2 Le suivi des déplacements entre les îles d'espèces envahissantes (dans les ports et autres points d'entrée) équivaut à la surveillance de la biosécurité et est couverte par l'objectif C1.4 ci-dessous. 


\section{B2: Prioritisation}

Établir et mettre en œuvre des systèmes efficaces d'évaluation des risques et de priorisation des espèces envahissantes, des voies d'introduction et des sites à gérer.

\section{Justification}

La plupart des îles contiennent un nombre important et croissant d'espèces introduites, délibérément ou accidentellement, à partir d'autres endroits. Certaines d'entre elles sont précieuses (plantes médicinales, de cultures, ornementales, etc.) alors que d'autres ont de graves répercussions. Souvent, le groupe le plus important est constitué d'espèces qui ne causent pas encore de problèmes sur une île donnée, mais qui peuvent devenir envahissantes à l'avenir, y compris les espèces introduites récemment, les plantes dans les jardins et les animaux domestiques. L'analyse des risques, y compris l'évaluation du risque des adventices ou phytosanitaire et l'analyse des voies d'introduction, a trois fonctions principales: déterminer s'il faut autoriser ou refuser l'introduction d'une nouvelle espèce dans un pays ou dans une île; déterminer l'importance des voies d'introduction des espèces nuisibles connues; et déterminer quelles espèces, parmi la totalité des espèces déjà introduites sur une île, peuvent devenir de sérieux problèmes futurs. La gestion de différents sites ou îles nécessite également d'être priorisée en fonction de leur diversité biologique ou d'autres valeurs. Des systèmes d'évaluation objective sont disponibles pour faciliter la prise de telles décisions, mais sont sous-utilisés.

But

Les planificateurs et les gestionnaires utilisent des outils d'aide à la décision efficaces pour déterminer objectivement les risques liés aux espèces envahissantes et pour prioriser la gestion des espèces, des voies d'introduction, des îles et des sites. Les ressources sont consacrées à la gestion des espèces causant le plus de dégâts, des espèces et des voies d'introduction présentant les risques les plus importants, ainsi que des sites les plus menacés et où l'action est susceptible d'être la plus efficace et la plus réalisable.

\section{Objectif}

B2.1: Concevoir, développer et adopter des systèmes simples et efficaces d'évaluation des risques et de priorisation pour les îles.

\section{Actions}

B2.1a Utiliser l'analyse des voies d'introduction pour identifier les voies d'introduction des espèces non indigènes et les prioriser pour être gérées.

B2.1b Adopter ou adapter des modèles existants pour évaluer le risque d'envahissement des espèces et les appliquer à (i) la prise de décision pour le contrôle des importations et des frontières et (ii) la détermination de la probabilité que les espèces introduites établies deviennent envahissantes.

B2.1c Utiliser l'évaluation des risques pour le contrôle des frontières afin de sélectionner les espèces dont l'importation devrait être autorisée dans une île ou dans un pays.

B2.1d Utiliser l'évaluation des risques pour évaluer toutes les espèces introduites déjà présentes sur une île, en tenant compte des informations disponibles du monde entier sur chaque espèce et des informations de l'île en question sur son comportement local, et utiliser les résultats pour prioriser les espèces à gérer.

B2.1e Utiliser et adapter des outils de priorisation pour les îles ou pour les sites sur la base de leur biodiversité et d'autres valeurs (p. ex., la base de données "Threatened Island Biodiversity » sur la biodiversité des îles menacées : voir Ressources p. 42), pour prioriser les actions sur différents sites ou îles.

\section{B3: Recherche sur les priorités}

Comprendre les espèces envahissantes prioritaires, y compris leur biologie et leurs impacts, et développer des techniques de gestion efficaces.

\section{Justification}

La priorisation identifie les espèces, les sites et les voies d'introduction pour une action de gestion immédiate. Elle peut également identifier les priorités potentielles pour lesquelles d'autres recherches sont nécessaires pour confirmer leur importance, notamment la recherche sur les impacts possibles. Une gestion efficace exige une connaissance de l'écologie, de la dynamique des populations et des mécanismes de transport des espèces cibles, ce qui pourrait nécessiter des recherches plus approfondies, car il se peut que des techniques efficaces de gestion d'une espèce ou d'une voie d'introduction n'existent pas et doivent être mises au point. La recherche axée sur les priorités est nécessaire pour rassembler les connaissances requises pour une gestion efficace, tandis que la recherche sur l'écologie et les impacts des espèces introduites consolide la hiérarchisation des priorités.

\section{But}

Les programmes de recherche visent à fournir les informations nécessaires pour une gestion efficace et pour une évaluation des risques et une priorisation bien fondées. 
B3.1: Effectuer des

recherches sur les impacts, l'écologie, la biologie, les voies d'introduction et la gestion des espèces envahissantes hautement prioritaires et des espèces introduites mal connues.
B3.1a Élaborer des plans de recherche régionaux et nationaux sur les espèces envahissantes sur la base d'une priorisation objective.

B3.1b Étudier l'écologie, la biologie, la dynamique des populations et les voies d'introduction des espèces envahissantes prioritaires.

B3.1c Étudier l'écologie et les impacts actuels et potentiels des espèces introduites dont les répercussions sont non confirmées, mais soupçonnées graves.

B3.1d Revoir les techniques de gestion disponibles pour les espèces envahissantes prioritaires, tester les techniques existantes et en développer de nouvelles si nécessaire pour une gestion efficace.

B3.1e Développer des méthodes de contrôle innovantes, telles que les techniques de gestion génétique des ravageurs pour améliorer l'efficacité de la gestion (Harvey et al. 2017). 


\section{Justification}

Empêcher que les espèces ne traversent les frontières, en particulier les frontières naturelles entre les îles isolées par l'océan, est le moyen le plus rentable de gérer la menace qu'elles représentent : les coûts par espèce sont plus faibles pour la prévention que pour la gestion des espèces établies (voir Comment pp. 34-35). La prévention évite également les impacts d'une espèce alors que la gestion après établissement n'a souvent lieu qu'après les impacts. La prévention fonctionne en identifiant les voies d'introduction et en établissant quatre barrières : 1) le contrôle avant exportation vise à empêcher l'exportation d'espèces envahissantes connues depuis les endroits où elles sont établies vers les îles ; 2) le contrôle avant la frontière réglemente l'importation dans une île ou dans un pays ; 3) le contrôle à la frontière vise à empêcher l'arrivée d'espèces sur l'île ; 4) la détection précoce et la réaction rapide post-frontalières visent à détecter et à éradiquer complètement les espèces nouvellement arrivées avant qu'elles ne se propagent bien au-delà du point d'arrivée, car le traitement est alors moins onéreux et plus facile. Un système de biosécurité efficace doit inclure les quatre éléments. Les systèmes de prévention doivent également viser à être exhaustifs et ne pas se concentrer uniquement sur les ravageurs agricoles.

\section{But}

Des systèmes efficaces sont en place dans les îles du monde entier pour réglementer les introductions intentionnelles et pour détecter et gérer les introductions non autorisées ou accidentelles à travers les frontières internationales et entre les îles.

\section{Objectif}

\section{C1.1: Contrôle pré-export.}

Élaborer et mettre en œuvre des procédures et des méthodes améliorées d'inspection, de traitement, d'emballage et de transport pour les moyens de transport et les marchandises quittant les pays et les îles où des espèces envahissantes prioritaires sont présentes.

\section{Actions}

C1.1a Promouvoir le développement et l'adoption de normes internationales plus strictes régissant la lutte contre les espèces potentiellement envahissantes à l'exportation.

C1.1b Utilisez l'analyse des risques et des voies d'introduction (partie B2 ci-dessus) afin d'identifier les espèces envahissantes prioritaires qui menacent vos îles en raison de leur présence chez les partenaires commerciaux et dans les moyens de transport atteignant vos îles, et d'identifier leurs voies d'arrivée.

C1.1c Revoir les procédures d'inspection et de traitement préalables à l'exportation pour contrôler les voies d'introduction prioritaires et empêcher l'exportation d'espèces envahissantes prioritaires, en particulier chez les principaux partenaires commerciaux de vos îles et dans les îles infestées qui fournissent les îles relativement vierges, et identifier les lacunes du champ d'application.

C1.1d Renforcer, développer et mettre en œuvre des contrôles de pré-exportation adéquats visant à prévenir l'exportation d'espèces envahissantes prioritaires.
C1.2: Contrôle pré-frontalier. Appliquer un processus rigoureux d'analyse des risques (parties B1.3 et B2 ci-avant) pour permettre ou interdire l'introduction délibérée d'espèces et la circulation des marchandises potentiellement porteuses entre pays et entre îles au sein d'un pays, et mettre en place des contrôles sur les navires de transport vers les îles, pour éviter les introductions accidentelles.
C1.2a Établir et mettre en œuvre une évaluation nationale et interne (interinsulaire) des risques et des voies d'introduction (parties B1.3 et B2 ci-dessus) pour les déplacements proposés d'espèces et pour la circulation de marchandises susceptibles de transporter accidentellement des espèces envahissantes.

C1.2b Faciliter une approche globale, qui suit le principe de précaution, pour la prise de décision en matière d'introductions proposées, dans la mesure du possible sur la base de listes positives (parfois appelées "listes blanches ") d'espèces classées comme (1) espèces " permises » à faible risque, (2) espèces " restreintes » à risque modéré et (3) espèces « interdites » à haut risque, et l'interdiction automatique de tout organisme ou marchandise ne figurant pas sur les listes permises ou restreintes.

C1.2c Coopérer pour promouvoir, appliquer et faire respecter les mécanismes internationaux et nationaux de traitement des marchandises et des navires de transport en transit, tels que la Convention internationale pour le contrôle et la gestion des eaux de ballast et des sédiments des navires, les protocoles de traitement de l'encrassement des coques, la lutte antiparasitaire sur les navires et les traitements insecticides dans les avions. 
C1.3: Contrôle à la frontière.

Établir et maintenir des systèmes efficaces de quarantaine et de contrôle des frontières et de transport, aux frontières nationales et entre les îles au sein d'un même pays.

\section{C1.4: Détection précoce} et réaction rapide postfrontalière.Établir et maintenir des systèmes efficaces pour détecter les incursions d'espèces envahissantes de manière fiable et rapide, et mettre en place des interventions d'éradication rapides.
C1.3a Examiner les contrôles aux frontières, les contrôles de transport et les systèmes de quarantaine existants pour identifier les lacunes dans le contrôle des voies d'introduction prioritaires, des vecteurs (navires, avions, personnes, organismes porteurs, transporteurs, etc.) ou des taxons (c.-à-d., toutes les espèces envahissantes, pas seulement des parasites agricoles), mais aussi les contraintes techniques ou en matière de ressources par rapport à la mise en œuvre de la quarantaine.

C1.3b Sensibiliser les citoyens et les visiteurs aux menaces relatives aux espèces envahissantes et aux réglementations sur la biosécurité, en utilisant des panneaux, des dépliants ou des vidéos dans les avions, les ferries entre les îles et aux points d'entrée. Mettre à disposition des contenants pour l'élimination des articles interdits.

C1.3c Mettre en place et renforcer les systèmes de contrôle de la quarantaine terrestre, d'eau douce et marine aux frontières et aux points d'entrée des îles, en veillant à ce que des organisations compétentes contrôlent les frontières à travers des agents ayant bénéficié d'une formation large (pas seulement dans l'agriculture), qui savent reconnaître les espèces envahissantes et leurs voies d'introduction, ayant le pouvoir d'intercepter, de confisquer et d'éliminer, ayant accès à des infrastructures adéquates de détection, de stockage et d'élimination, et permettant une collecte et une gestion des données exhaustives.
C1.4a Développer des centres d'information régionaux pour suivre la propagation des espèces introduites et rendre disponibles les mises à jour relatives à l'état et à la distribution depuis chacune des îles.

C1.4b Examiner les dispositifs existants de surveillance portuaire et frontalière et de réaction rapide et identifier les lacunes de la gestion nationales, insulaires, taxonomiques ou des voies d'introduction, en particulier par rapport aux espèces et aux voies d'introduction à haut risque identifiées par l'évaluation des risques (parties B2 ci-dessus).

C1.4c Concevoir et mettre en œuvre des systèmes de surveillance aux points d'entrée et aux sites d'introduction (pépinières, fermes expérimentales, etc.) en utilisant les meilleurs techniques en la matière, notamment des plans de surveillance et des outils d'identification propres aux espèces et aux voies d'introduction à risque élevé, et des protocoles de déclaration spécifiques lorsque des organismes sont détectés.

C1.4d Élaborer des programmes de science citoyenne pour le suivi et le signalement des espèces « inconnues » en général et des espèces ciblées, tels que les réseaux d'apiculteurs pour les hyménoptères.

C1.4e Développer et mettre en place des plans de réaction rapide avec du personnel formé, du financement dédié et de l'équipement prêt à l'emploi, pour faire face aux différents types de ravageurs nouvellement arrivés, tout en mettant l'accent sur l'éradication rapide. Tester les plans en effectuant des essais sur place.

\section{C2: Gestion des espèces envahissantes établies}

Réduire ou éliminer les impacts des espèces envahissantes établies, par l'éradication, la lutte biologique, le confinement, l'exclusion, le contrôle physico-chimique ou l'atténuation des impacts.

\section{Justification}

La gestion des espèces envahissantes installées dans les îles souffre d'un manque de ressources par rapport à l'ampleur du problème, et les ressources sont souvent consacrées au contrôle à long terme, tandis qu'un investissement initial important dans l'éradication ou la lutte biologique pourrait produire un résultat plus rentable à long terme. Les résultats des projets de gestion sont souvent mal consignés, de sorte que leur rapport coûtefficacité ne peut être estimé et que les résultats ne sont souvent pas rendus publics, ce qui limite la capacité d'apprendre de l'expérience des autres.

But

Pour assurer une rentabilité à long terme, une approche hiérarchique du choix du but de gestion est adoptée (voir Comment sélectionner les objectifs de gestion, pp. 34-35). Pour garantir l'optimisation des ressources, 
tous les projets de gestion mettent en place un suivi de l'efficacité opérationnelle (succès à atteindre le contrôle de l'espèce) et des résultats du projet (succès dans la protection de la biodiversité ou d'autres valeurs). Les résultats sont diffusés pour permettre l'amélioration des pratiques de gestion. Les impacts des espèces envahissantes établies sont réduits ou éliminés au moyen d'une éradication efficace, de la lutte biologique, du confinement, de l'exclusion, du contrôle physico-chimique de la population ou de l'atténuation des impacts.

\section{Objectif}

C2.1: Concevoir, adopter et utiliser des outils de décision en vue d'orienter le choix des buts de gestion et des techniques de gestion, afin d'assurer la rentabilité et l'utilisation efficace de ressources limitées.

\section{Actions}

C2.1a Concevoir, adopter et utiliser des outils de décision qui évaluent la faisabilité et les coûts des différentes options de gestion, en vue d'orienter le choix du but de gestion (voir Comment sélectionner les buts de gestion pp. 34-35, Cacho et Pheloung 2007, IPMDAT: Ressources p. 43) et les techniques utilisées pour atteindre ce but.
C2.2: Concevoir et mettre en œuvre des programmes de gestion efficace adaptés à chaque espèce et à chaque site, en incorporant des normes de meilleures pratiques (voir Ressources pp. 42-43).
C2.2a Concevoir et mettre en œuvre tous les projets de gestion selon les meilleures pratiques en vigueur (voir Ressources p. 42), selon les normes de sécurité les plus élevées en matière de santé humaine, de bien-être animal et d'effets non ciblés (IUCN 2017a), et avec la pleine participation de toutes les parties concernées (y compris les communautés locales).

C2.2b S'assurer que tous les projets de gestion intègrent le suivi des résultats, une gestion efficace des données et une évaluation des succès et des résultats, afin de faciliter une gestion adaptative et d'intégrer autant que possible la restauration de la biodiversité ou d'autres valeurs (partie C3 ci-dessous) dans les projets de gestion.

C2.2c Concevoir et mettre en œuvre des projets d'éradication d'espèces pour lesquelles l'éradication est jugée possible avec des ressources accessibles (voir Comment p. 34-35, Ressources p. 43), en particulier pour les nouvelles incursions ou sur les petites îles, y compris l'éradication multi-espèces pour une efficacité accrue et la prévention des effets de la libération de niches écologiques et l'augmentation conséquente de l'abondance d'autres espèces envahissantes.

C2.2d Concevoir et mettre en œuvre des projets de lutte biologique contre les espèces appropriées, en particulier les espèces largement répandues qui sont difficiles à gérer par d'autres moyens.

C2.2e Concevoir et mettre en œuvre un contrôle par des techniques de gestion génétique telles que le mâle stérile, l'incorporation de transgène, etc., lorsque cela est jugé possible, conforme aux normes internationales et socialement acceptable (voir Harvey et al. 2017).

C2.2f Concevoir et mettre en œuvre des projets de confinement pour les espèces dans des situations appropriées, telles que les arrivées récentes, ou les cultures valorisées qui sont également envahissantes.

C2.2g Concevoir et mettre en œuvre des projets d'exclusion dans les situations appropriées, y compris l'exclusion de plusieurs espèces envahissantes des sites de hautes valeurs pour la conservation en utilisant les barrières multi-espèces pour isoler une partie du territoire d'une île.

C2.2h Concevoir et mettre en œuvre des projets efficaces de contrôle physique ou chimique dans les situations appropriées, en particulier les sites de grande valeur envahis par une variété d'espèces introduites.

C2.2i Lorsque les impacts sont causés par une espèce envahissante qui n'est pas susceptible à un contrôle direct efficace, concevoir et mettre en œuvre les mesures d'atténuation, y compris la protection de la biodiversité de grande valeur par des moyens tels que la protection des nids ou le maintien de la population ex-situ. 


\section{Justification}

Parfois, le contrôle d'une espèce envahissante est suivi d'une récupération rapide et adéquate de l'écosystème indigène ou de la valeur économique ou sociétale affectée par l'espèce cible. Mais dans d'autres cas, les espèces indigènes peuvent ne pas se reconstituer ou des conséquences néfastes imprévues peuvent survenir, telles que l'invasion par d'autres espèces introduites. Dans de tels cas, une intervention supplémentaire peut être nécessaire pour aider au rétablissement de la biodiversité indigène ou d'autres valeurs. Cela peut inclure des projets de restauration particuliers propres à chaque espèce indigène, ou la gestion d'autres espèces envahissantes.

But

Les espèces indigènes, les écosystèmes, les services écosystémiques et les autres valeurs affectées retrouvent leurs niveaux prévus à la suite de la gestion des espèces envahissantes.

\section{Objectif}

C3.1: Concevoir et mettre en œuvre des projets de restauration post-gestion pour veiller à ce que les projets de gestion des espèces envahissantes atteignent leur but ultime, soit le rétablissement des espèces et des écosystèmes indigènes des services écosystémiques ou d'autres valeurs économiques ou sociétales. $\checkmark$ Actions

C3.1a Veiller à ce que tous les programmes de gestion des espèces envahissantes soient accompagnés et suivis d'une surveillance et d'une évaluation à long terme des résultats, y compris le rétablissement attendu des espèces indigènes, des écosystèmes et d'autres valeurs affectées.

C3.1b Veiller à ce que des mesures soient prises pour limiter d'autres pressions sur la biodiversité indigène, l'écosystème ou d'autres valeurs, y compris la biosécurité afin de prévenir de nouvelles invasions.

C3.1c Lorsque la gestion des espèces envahissantes est suivie d'autres invasions, concevoir et mettre en œuvre des projets de gestion de ces autres espèces envahissantes, au besoin.

C3.1d Lorsque d'autres interventions sont jugées nécessaires pour assurer le plein rétablissement de la biodiversité ou d'autres valeurs après la gestion des espèces envahissantes, concevoir et mettre en œuvre les projets visant à restaurer les espèces clés (y compris les espèces menacées), les habitats ou les caractéristiques du paysage, au besoin. 


\section{Comment...}

1. ... impliquer, créer une dynamique et susciter des soutiens
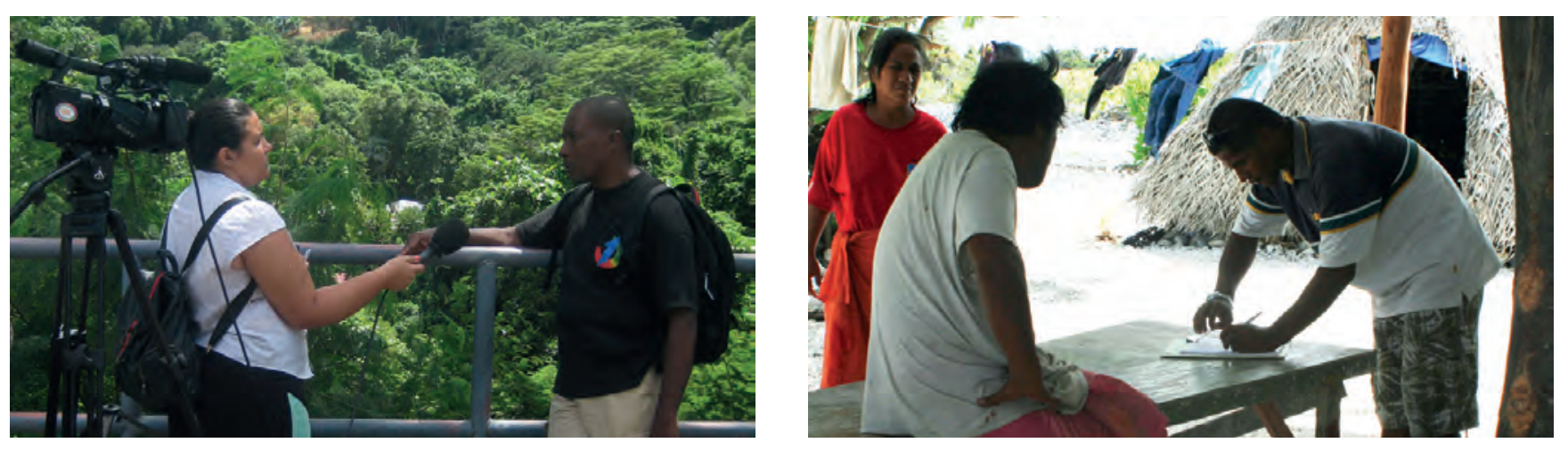

2. ... rédiger le plan, prioriser, prendre des décisions
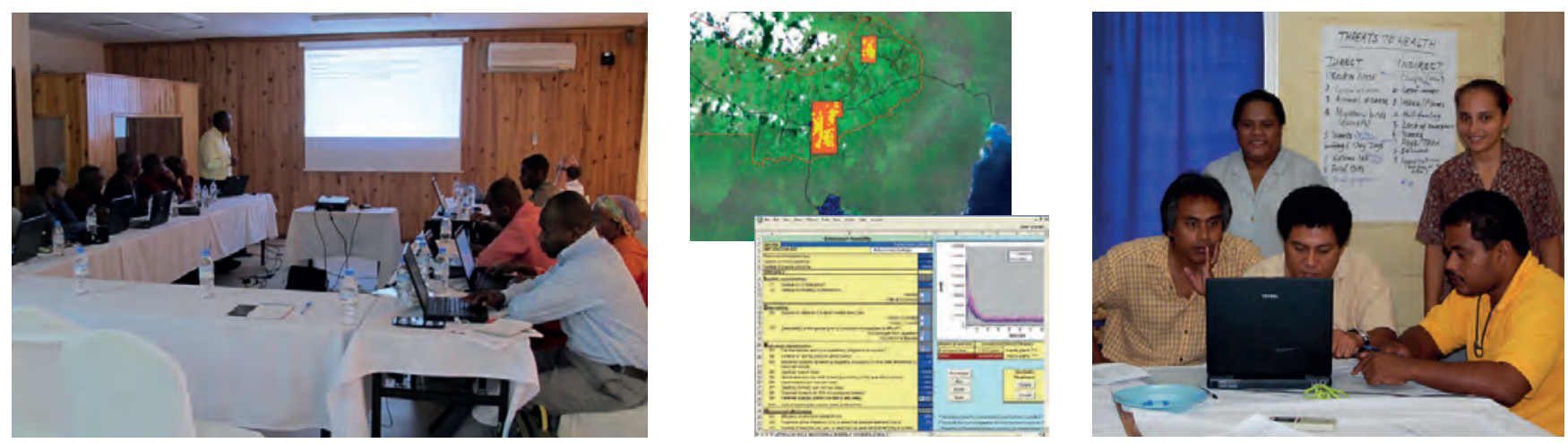

3. ... passer des plans aux actions
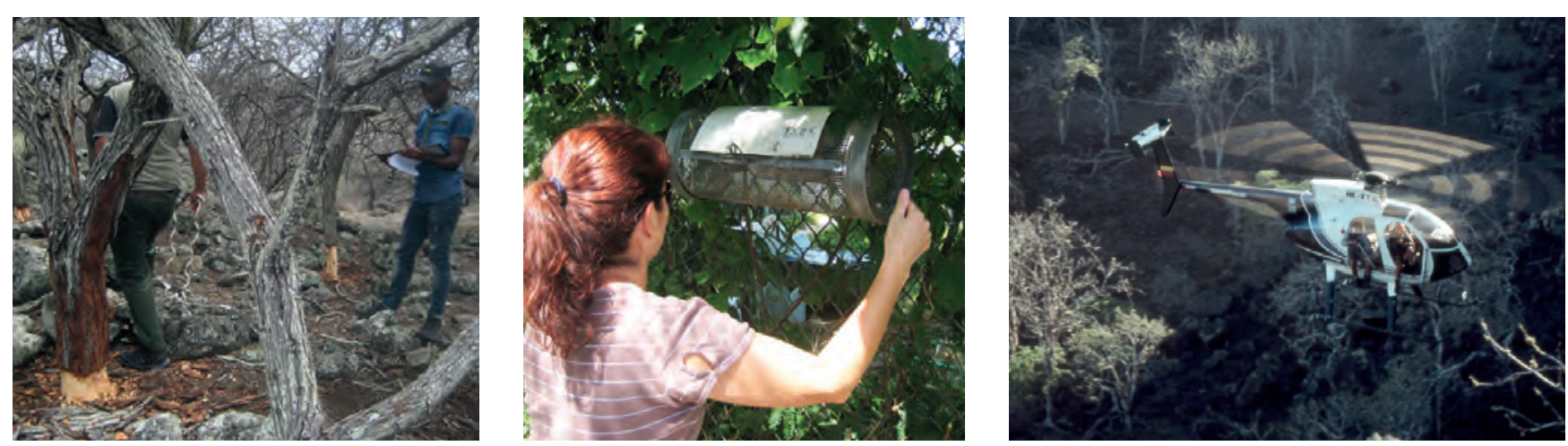


\title{
1. Comment impliquer, créer une dynamique et susciter des soutiens
}

\section{Impliquer toutes les strates de la société}

\begin{abstract}
Les gouvernements insulaires ne peuvent pas assumer la responsabilité de toutes les activités requises pour prévenir et gérer les espèces envahissantes; ils ont besoin de l'aide et de la compréhension du public. Idéalement, les gouvernements, les ONG, le secteur privé et le grand public devraient partager les tâches de prévention et de gestion des espèces envahissantes. Les gouvernements des îles n'ont généralement pas assez de compétences techniques ni de ressources et ne sont souvent pas pleinement conscients des dommages que les espèces envahissantes peuvent causer aux moyens de subsistance et au développement. Une prise de conscience publique et politique accrue des problèmes causés par les espèces envahissantes est nécessaire afin de générer du soutien à l'égard de la lutte contre ces dernières.
\end{abstract}

Une stratégie de communication peut aider. Mettre l'accent sur l'éducation des personnes pour qu'elles comprennent les dégâts causés par les espèces envahissantes et ce qu'elles peuvent faire pour y faire face. La publicité et la persuasion doivent cibler les décideurs, les communautés locales, les ONG et les entreprises, mais aussi les enfants (afin de développer leur conscience sociale en pensant à demain). Vous essayez de changer leur comportement, et donc les arguments utilisés pour chaque public cible peuvent varier grandement : p. ex., un argument poignant pour les décideurs et les donateurs pourrait être que la gestion des espèces envahissantes augmente la résilience des écosystèmes au changement climatique, alors que les agriculteurs réagiront mieux aux informations sur les envahisseurs qui pourraient bientôt affecter leurs exploitations. Les outils de communication utilisés (p. ex. les affiches, les bulletins d'information, les articles dans la presse locale, les interviews à la radio et à la télévision, les sites Web, les blogues, les médias sociaux...) varieront également selon le public ciblé, p. ex., la radio et la télévision seront utilisées pour les communautés locales qui ne lisent pas beaucoup ou qui n'utilisent pas Internet. Rappelez-vous que les gens des médias ont généralement besoin d'aide afin d'assurer qu'ils expliquent correctement les informations et en termes clairs.

Essayez de motiver et de promouvoir la gestion environnementale : les supports tels que les affiches, les sites Web et les messages publicitaires télévisés peuvent inclure des parties parlant de " ce que vous pouvez faire pour aider à combattre cette menace » ou « mon rôle dans la lutte contre cet envahisseur ». Encouragez les personnes à signaler les observations, à ne pas propager de plantes envahissantes lors d'activités liées à l'agriculture ou au jardinage, et à contribuer à la gestion des espèces envahissantes proche de chez soi. La prise de conscience des risques et des coûts des espèces envahissantes ainsi que des meilleurs moyens de les gérer peut rapidement amener les communautés locales, les ONG et les entreprises à s'impliquer dans les prises de décisions concernant les espèces envahissantes, à les prévenir et à les gérer. Là où l'intérêt et le soutien local sont déjà existants, les citoyens et les écoliers peuvent s'impliquer dans les programmes de « science citoyenne » pour la collecte d'informations, notamment la détection de nouvelles invasions et les suivis qui complémentent ceux effectués par les organisations techniques. Les applications pour smartphones fonctionnant avec un appareil photo et un GPS permettent de télécharger les photos vers un serveur et de les identifier rapidement (p. ex. PlantNet : voir Ressources p. 42). Un bon exemple de participation des citoyens est l'initiative Naturewatch sur le site Web iNaturalist en Nouvelle-Zélande (<http://inaturalist.nz/>). Les citoyens peuvent s'inscrire en ligne et signaler n'importe quelle espèce qu'ils ne reco nnaissent pas, par un formulaire. De telles initiatives peuvent être efficaces, et la participation peut être très satisfaisante pour les citoyens impliqués, surtout s'ils voient les résultats de leurs propres efforts, et leur nom dans les médias.

Là où la sensibilisation est la plus grande, les communautés réalisent elles-mêmes certains aspects de la prévention et de la gestion des espèces envahissantes. De nombreuses îles ont des groupes communautaires qui gèrent des actions telles que le recyclage et le nettoyage des plages, et avec une sensibilisation accrue aux enjeux des espèces envahissantes, ces groupes peuvent également prendre en charge la gestion des espèces envahissantes.

Sur les deux pages qui suivent, se trouvent quelques points importants à garder à l'esprit lorsqu'on communique avec le grand public et les médias au sujet des espèces envahissantes. 


\section{Messages clés de sensibilisation}

\section{Utilisez un langage simple et non ambigu ! (Rappelez-vous : pour la plupart des personnes, le mot “ exotique » signifie intéressant et désirable)}

En tant que professionnel qui travaille quotidiennement avec des espèces envahissantes, vous avez l'habitude d'utiliser le jargon technique. Lorsque vous vous adressez à un auditoire ou rédigez pour le grand public, que ce soit un groupe communautaire local, un propriétaire foncier, une réunion avec des représentants du gouvernement, une interview ou un article pour les médias, n'utilisez pas ces termes techniques à moins d'être prêt à expliquer chacun d'eux, plus d'une fois, et de souffrir malgré tout d'incompréhension (peu importe le nombre de fois que vous l'expliquerez, les personnes continueront à mal interpréter le jargon). Dans les documents et les campagnes de sensibilisation du public, et en particulier lors des consultations menées auprès des parties prenantes, évitez d'utiliser des termes techniques ou chargés d'une autre signification tels que « exotique ", " envahissant », " biodiversité ", qui peuvent vous être très familiers en tant que professionnel engagé dans la lutte contre les espèces envahissantes, mais que la plupart des personnes ordinaires ne comprennent pas vraiment. Mettez-vous à leur place : pour la plupart des personnes, le mot « exotique » évoque quelque chose de plaisant ou de rêve. Dans le langage courant, le mot "envahissant » n'est pas associé à des impacts particuliers au contraire d'autres termes communs comme " parasite » ou " ravageur ». Donc, utilisez un langage simple : dites « nuisibles » ou « mauvaises herbes » au lieu d'envahissantes, dites " introduites » ou « non indigènes » au lieu d'exotiques, et dites " nature », « faune » ou « nos propres espèces indigènes » (selon le contexte) au lieu de biodiversité. Les documents de sensibilisation peuvent permettre aux personnes d'apprendre des termes techniques, mais partez toujours du principe que les personnes ne les connaissent pas, donc adaptez votre langage au leur.

\section{Quelles espèces sont introduites ou indigènes ? (La plupart des personnes ne le savent pas !)}

La gestion des espèces envahissantes et le suivi des espèces introduites sont entravés par le fait que la plupart des personnes ne savent pas très bien quelles espèces sont indigènes et lesquelles ont été introduites. Sur la plupart des îles, les personnes (y compris les politiciens) ne sont tout simplement pas conscientes que les "forêts " et les jardins qui les entourent sont principalement constitués d'espèces introduites et envahissantes. En conséquence, les personnes n'apprécient pas leurs plantes et leurs animaux indigènes et ne réalisent pas les dégâts causés par les espèces envahissantes. Les îles ont besoin de listes d'espèces indigènes, introduites et envahissantes (couvrant tous les groupes taxonomiques) et de matériels publicitaires pour aider les personnes à les reconnaître et à les traiter de manière appropriée. Les matériels et les campagnes peuvent être basés sur des espèces envahissantes communes que les personnes reconnaissent, sans savoir qu'elles sont introduites, ainsi que sur la valeur (culturelle, économique) des espèces authentiquement indigènes. Vous pouvez alors encourager les personnes à éviter d'utiliser des espèces envahissantes dans la mesure du possible (comme certaines plantes de jardin, p. ex.), et à utiliser plutôt des espèces indigènes.

Quels sont les impacts des espèces envahissantes et quels sont les avantages de les gérer? (Mis à part les agriculteurs, la plupart des personnes n'en ont qu'une vague idée...)

Mettez l'accent sur les espèces envahissantes qui ont le plus d'impact sur les personnes. La plupart des gens ne se soucient pas des espèces envahissantes qui leur apportent également des avantages. Montrez comment la gestion des nuisibles pourrait réduire leurs impacts, et utilisez des catastrophes connues liées aux ravageurs pour promouvoir les efforts de biosécurité - l'arrêt de l'introduction des parasites dans l'île empêchera que de telles catastrophes se reproduisent à l'avenir. Utilisez des exemples de réussite : montrez comment il est possible de faire la différence positive, grâce à des exemples d'éradications, de la lutte biologique ou des autres projets réussis. Essayez de convaincre les électeurs et aidez les groupes communautaires à convaincre leurs politiciens de faire quelque chose à ce sujet. Vous pouvez également utiliser des études coûts-bénéfices pour faire passer le message à propos des avantages de la prévention et de la gestion rapide une fois l'espèce introduite pour expliquer que $1 €$ dépensé aujourd'hui représente $100 €$ économisés à l'avenir. 


\section{L'introduction d'espèces envahissantes n'accroît pas la biodiversité !}

Attendez-vous à contrer l'argument selon lequel l'introduction de tout un tas de nouvelles espèces dans votre île est une bonne chose « parce qu'elle augmente la biodiversité ». La biodiversité est un concept global, tandis que l'augmentation de la diversité locale en introduisant des espèces qui sont communes ailleurs peut en fait réduire la biodiversité mondiale en provoquant l'extinction d'espèces endémiques qui ne se trouvent que sur votre île et nulle part ailleurs dans le monde.

\section{Tout le monde a un rôle à jouer}

Les espèces envahissantes sont un problème dans presque toutes les îles du monde et cela n'est pas la faute du gouvernement ou de n'importe quel autre groupe, mais résulte d'un comportement mal informé, malavisé ou insensible dans l'ensemble de la société. Mais rappelez aux personnes que l'action individuelle n'est pas sans effet - chaque citoyen peut combattre les espèces envahissantes avec ses propres décisions et comportements. Quelles sont les plantes qu'ils choisissent pour leur jardin, quels animaux de compagnie ils choisissent de garder et comment ils s'en occupent, comment ils gèrent leurs terres - autant de décisions que chacun peut prendre de façon responsable. La façon dont ils votent, les organisations environnementales auxquelles ils donnent de l'argent ou du temps, et ce qu'ils enseignent à leurs enfants, amis et voisins, ont tous un effet au-delà de leur propre personne. Essayez de convaincre les personnes du « pouvoir de l'individu » dans les campagnes, p. ex. faites-leur comprendre les dangers de rapporter de leurs vacances des produits animaux ou végétaux et des semences; envisagez un code de conduite volontaire pour de telles choses. Et commencez par donner l'exemple : en dehors de votre travail de lutte contre les espèces envahissantes, évitez de les planter dans votre jardin, de les garder comme animaux de compagnie, ou d'apporter les fruits des espèces envahissantes sur des îles où ils ne sont pas présents!

\section{Clients difficiles}

\section{Comment surmonter les problèmes liés aux "conflits d'intérêts " ou aux techniques de gestion impopulaires}

L'organisme nuisible d'une personne est la nourriture, l'animal de compagnie ou la jolie plante de jardin d'une autre personne. Chaque région océanique a des espèces comme celle-ci. Les porcs sont l'une des espèces les plus controversées du Pacifique en raison de leur importance pour l'alimentation des populations insulaires et de leur empreinte historique, mais aussi de leurs impacts énormes sur les écosystèmes insulaires en modifiant la structure des forêts et la composition des espèces d'arbres et en devenant les prédateurs des animaux indigènes, y compris des œufs de tortues de mer, des oiseaux de mer nichant au sol et des oiseaux terrestres incapables de voler. Localement, chaque île peut avoir ses propres cas particuliers, tels que les macaques introduits à l'île Maurice, prédateurs notoires de la faune endémique, mais protégés par certaines personnes et même promus comme attraction touristique.

Un autre type de conflit porte sur l'acceptabilité publique des techniques de gestion. La gestion de la plupart des espèces envahissantes consiste à les tuer, ce que la plupart des personnes n'aiment pas, même les professionnels de la conservation qui ne veulent pas utiliser certains techniques, en raison de leurs propres croyances et de la peur des réactions publiques. Ce type d'opposition peut être exprimé violemment par des groupes de défense des droits des animaux et devient beaucoup plus fort et plus répandu lorsqu'une technique de gestion consiste à tuer un grand nombre d'individus d'une espèce populaire (comme les macaques ou les chats) ou est perçue comme susceptible d'avoir des impacts directs sur la population humaine, p. ex., par l'ingestion d'herbicide dans l'eau potable ou par la consommation de poison par les animaux domestiques ou les enfants.

Traiter de telles situations nécessite évidemment minutie, temps et investissement. Dans certains cas, vous pourriez obtenir l'appui d'un politicien local influent pour faciliter un projet susceptible d'être impopulaire auprès du public (p. ex. éradiquer les chats sauvages, interdire certaines importations d'animaux), mais il sera souvent plus efficace d'opter pour une approche ascendante participative. 
Les points clés sont la persévérance, la patience, les relations personnelles, la sympathie et la compréhension. Cela peut prendre beaucoup de temps - voire des années - pour convaincre les personnes ordinaires, mais concentrez-vous d'abord sur elles. En particulier, identifiez les personnes clés affectées par le projet proposé ainsi que celles qui ont de l'influence (il se peut que ce ne soit pas les mêmes, alors vous aurez peut-être besoin de deux approches différentes). Méfiez-vous des réunions communautaires - vous devrez peut-être en organiser, mais ne comptez jamais sur elles pour changer l'opinion publique. Les réunions sur les controverses sont généralement dominées par une poignée de personnes qui pourront facilement contrer par la rhétorique vos arguments bien fondés, vous laissant avec encore moins de soutien qu'avant la réunion. Au lieu de cela, organisez les réunions de sorte à apprendre à connaître les personnes en leur posant des questions ; identifiez les opposants virulents et leurs préoccupations, et résistez à l'envie d'essayer de les persuader sur le coup. Ensuite, parlez aux personnes individuellement autant que possible. Apprenez à connaître les personnes, et parlez-leur encore et encore. Renseignez-vous sur leurs préoccupations et prenez-les au sérieux. Essayez de découvrir et de mieux comprendre les différences plus profondes entre les divers groupes d'une communauté, ce qui pourrait influencer leur jugement sur un problème donné. Réfléchissez bien à la meilleure façon d'influencer les objecteurs clés, soit en leur parlant directement soit en persuadant la majorité de les convaincre. Attendez-vous à découvrir que vos arguments clés initiaux peuvent ne pas avoir beaucoup de poids auprès des objecteurs, dans quel cas vous devrez peut-être trouver des raisons et des explications qu'ils pourront comprendre, identifier, accepter et soutenir. Adaptez vos techniques de gestion proposées pour faire face à des objections que vous ne pouvez pas surmonter en discutant.

Tout cela représente beaucoup de travail, mais si votre projet est hautement prioritaire, cela en vaut la peine. En fait, il est essentiel de garder à l'esprit qu'il sera difficile de convaincre quelconque donateur de payer pour un projet controversé, donc n'essayez pas tant que vous n'avez pas le soutien du public. Une fois que vous avez l'accord du public (y compris des politiques) pour le travail, il devient plus facile de trouver des fonds. Et avec le soutien des personnes locales que vous connaissez bien, la mise en œuvre du projet sera bien plus facile.

Les consultations sur l'éradication des rongeurs sur l'île Lord Howe sont un bon exemple des pièges et d'une conclusion éventuellement heureuse de ce processus : <http://hirodenteradicationproject.org/ the-project/community-engagement/>. Vous pouvez utiliser également l'organigramme pratique pour les consultations de planification, développé par Novoa et al. (2018 : voir la version simplifiée ci-dessous), et un guide sur la résolution des conflits développé par Madden et McQuinn (2014).

Étapes à suivre pour assurer une consultation et un engagement complets:

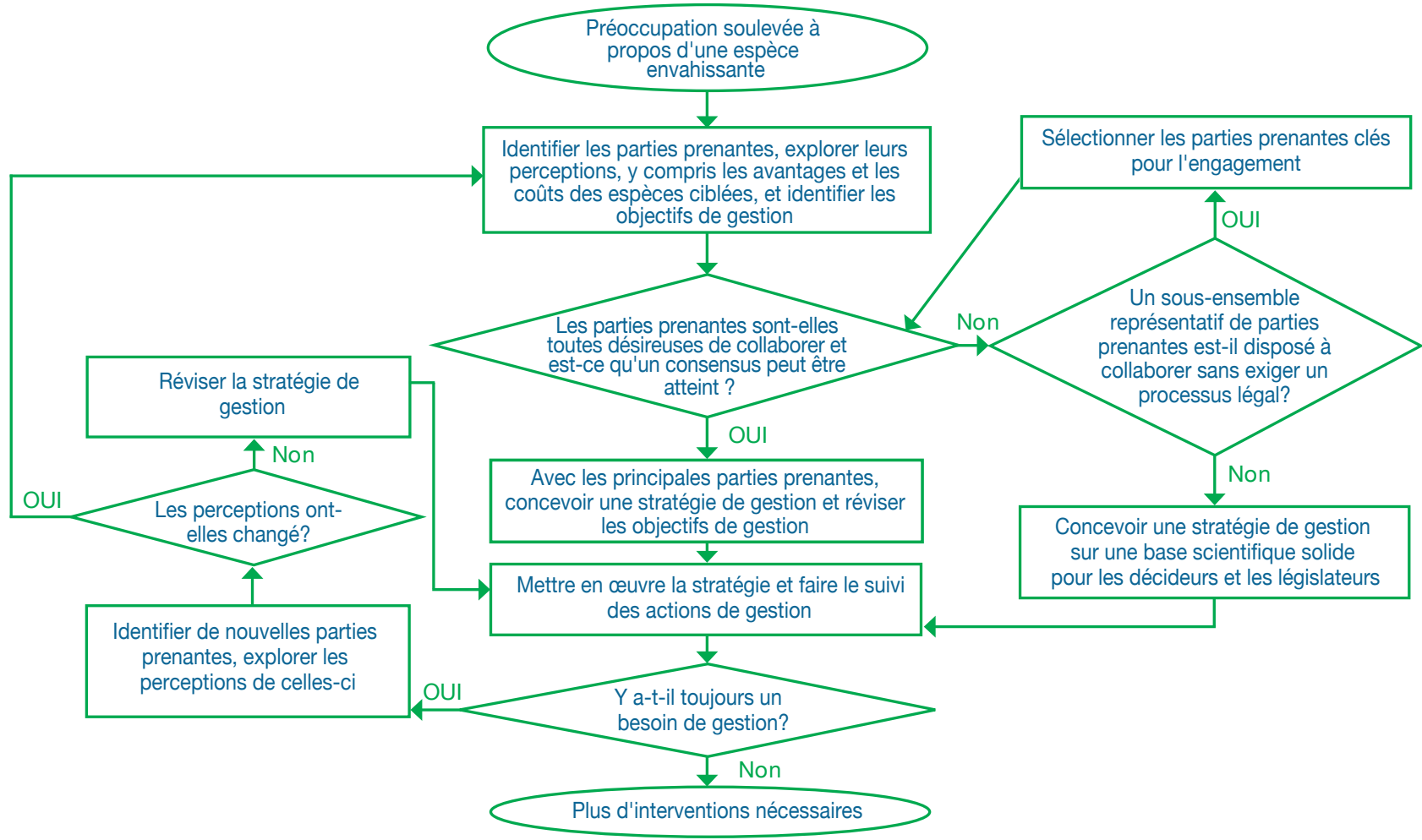




\section{Comment intégrer la problématique}

\section{Influencer au sommet}

II y a souvent une déconnexion entre les « techniciens » des départements gouvernementaux et des ONG et les décideurs politiques, et c'est souvent le plus grand obstacle à l'intégration de la gestion des espèces envahissantes aux programmes nationaux. Les gouvernements ont déjà leurs priorités, et si les espèces envahissantes n'y sont pas déjà incluses, vous demanderez au gouvernement de réduire l'importance de ses priorités annoncées publiquement afin de fournir des ressources pour lutter contre les espèces envahissantes. Pour cette raison, il est important d'intégrer les espèces envahissantes dans les priorités gouvernementales existantes (p. ex. la sécurité alimentaire) en travaillant dans le cadre du système d'établissement des priorités du gouvernement pour les inclure. Décidez comment harmoniser vos priorités avec les priorités et les plans existants de votre gouvernement et démontrez les synergies aux divers représentants du gouvernement. Dites au gouvernement ce que vous pouvez lui offrir (p. ex., former du personnel dans divers départements, rédiger des messages de sensibilisation, soutenir un consultant pour rédiger une SPAEE nationale, etc.) et ce que vous demandez (p. ex., examen et mise à jour de la législation en vigueur, recrutement d'agents supplémentaires pour la mise en œuvre des actions, extension d'un traité, etc.). Soyez précis - des demandes vagues de soutien ne seront que vaguement soutenues. Rappelez-vous que les plans législatifs habituels de 4 à 5 ans ne sont pas très utiles pour la planification à long terme, mais travaillez sur des programmes à long terme si nécessaire et apprêtez-vous à faire pression lorsque le gouvernement est sur le point de changer. Obtenir une SPAEE signé officiellement et publiquement approuvé par les politiciens de tous les partis réduira les probabilités de la voir disparaître quand un nouveau parti prendra le relai. Mais pour faire tout cela, vous avez besoin d'une oreille attentive à un niveau politique élevé - pas toujours facile à trouver, mais si vous y arrivez, l'influence de cette personne peut s'accompagner d'une augmentation significative des ressources pour votre programme. Essayez d'intégrer ces Directives dans les politiques nationales, car une fois qu'elles sont devenues un instrument politique accepté, vous pouvez les utiliser pour soutenir et renforcer vos plans.

\section{Intégrer la gestion des espèces envahissantes dans d'autres plans}

Prenez contact avec d'autres secteurs importants tels que la planification du développement durable, l'aménagement du territoire et des terres agricoles, le commerce, la gestion des transports et des ports, la gestion des aires protégées et le changement climatique, et travaillez avec eux pour intégrer les espèces envahissantes dans leurs plans de gestion. Une façon de le faire est de former un comité multisectoriel sur les espèces envahissantes (plus de détails ci-dessous, p. 25). Ne négligez pas le secteur de la conservation! Travaillez avec les départements et les ONG impliqués dans la conservation afin d'intégrer la gestion des espèces envahissantes dans les plans de gestion des zones protégées ainsi que dans les plans de gestion des espèces menacées. Certains gestionnaires de la conservation ne prennent toujours pas suffisamment au sérieux les espèces envahissantes pour inclure leur gestion dans la gestion générale des sites. Le secteur de la biosécurité peut également être difficile à pénétrer à cause de son intérêt historique pour les parasites agricoles uniquement. Essayez de faire en sorte que les contrôles de biosécurité couvrent un maximum d'espèces, même celles qui ne sont pas encore dites "envahissantes " (voir l'évaluation des risques, pp. 31-33).

\section{Intégration aux plans de changements globaux}

Plusieurs types de changements et de tendances au niveau mondial affectent les îles et favorisent les espèces envahissantes. Ils peuvent être utilisés pour rehausser le profil et l'importance accordés à la gestion des espèces envahissantes.

- Le changement climatique peut favoriser les espèces envahissantes en perturbant les écosystèmes, car de nombreuses espèces envahissantes sont préadaptées pour tirer profit des zones perturbées, y compris celles causées par des catastrophes « naturelles ». En outre, l'élévation de la température et les changements dans les régimes de précipitations peuvent affecter la végétation et les animaux indigènes, alors que les espèces envahissantes ont tendance à s'adapter facilement (une des caractéristiques qui les rend envahissantes). La sensibilisation à cette adaptabilité plaide fortement en faveur de l'intégration de la gestion des espèces envahissantes dans les programmes nationaux d'atténuation du changement climatique et d'adaptation à ce dernier. Les catastrophes « naturelles » peuvent augmenter avec le changement climatique, mais leur impact sur les espèces envahissantes peut être réduit en élaborant des plans visant à minimiser les perturbations de l'habitat et à restaurer la végétation indigène dans les zones dégradées. De plus, la gestion des espèces envahissantes peut aider à maintenir ou à restaurer l'intégrité des écosystèmes et ainsi accroître de manière générale la résilience au changement climatique. C'est un argument fort en faveur de l'intégration de la gestion des 
espèces envahissantes dans les programmes d'adaptation aux changements climatiques basée sur les écosystèmes, ces programmes étant non seulement écologiquement importants et rationnels, mais pouvant aussi donner accès à des financements considérables.

- Défrichage des terres. Le défrichage de vastes zones de végétation indigène pour la production de grandes cultures ou l'exploitation minière favorise également la propagation d'espèces envahissantes. Comme la gestion des espèces envahissantes est souvent nécessaire pour réduire la dégradation des terres, il est possible de plaider de manière convaincante pour l'inclusion de la gestion des espèces envahissantes dans les politiques d'aménagement du territoire.

- Transport. L'accroissement mondial du commerce, des voyages, des transports et du tourisme est le principal facteur de propagation des espèces qui peuvent devenir envahissantes. Le développement de nouvelles routes, voies ferrées, voies navigables et liaisons aériennes et maritimes accroît la circulation des personnes et des marchandises ainsi que le déplacement d'espèces envahissantes. De plus, la création de routes, de voies ferrées et de cours d'eau permet aux espèces envahissantes de se propager dans les zones perturbées qui les bordent. Plaidez pour l'amélioration des mesures en matière de biosécurité dans la planification des secteurs du transport, du commerce et du tourisme.

- Loisirs, jardinage, animaux de compagnie et Internet. L'augmentation du temps de loisir et l'expansion des installations touristiques entraînent une utilisation accrue de plantes ornementales non indigènes, tandis que le jardinage pour la nourriture et les remèdes à base de plantes augmente également la demande d'espèces moins communément utilisées. Grâce à Internet, il est possible de commander toutes sortes de graines, même des animaux vivants. Les commerces liés aux animaux de compagnie et au jardinage comptent maintenant parmi les principaux facteurs d'introduction de nouvelles espèces dans le monde entier. Plaidez pour le renforcement des systèmes de biosécurité et d'évaluation des risques pour les industries de l'horticulture, des animaux domestiques, du tourisme et pour l'interception des organismes envoyés par la poste..

\section{Aide mutuel - comment mettre en place des réseaux régionaux}

Malgré l'amélioration des communications électroniques, les personnes travaillant sur les espèces envahissantes dans les îles sont gênées par les grandes distances et les barrières océaniques qui limitent les contacts humains et le partage d'expérience d'un groupe à un autre. Des efforts supplémentaires sont nécessaires pour surmonter " l'isolement insulaire » en créant des groupes régionaux de coordination et d'action conjointe, mais aussi des réseaux pour échanger des nouvelles, des informations, des expériences, des alertes, des annonces d'emploi et des demandes d'assistance. De tels réseaux peuvent effectivement produire des collaborations et renforcer les capacités grâce aux échanges de personnel, et contribuer grandement à la résolution des problèmes.
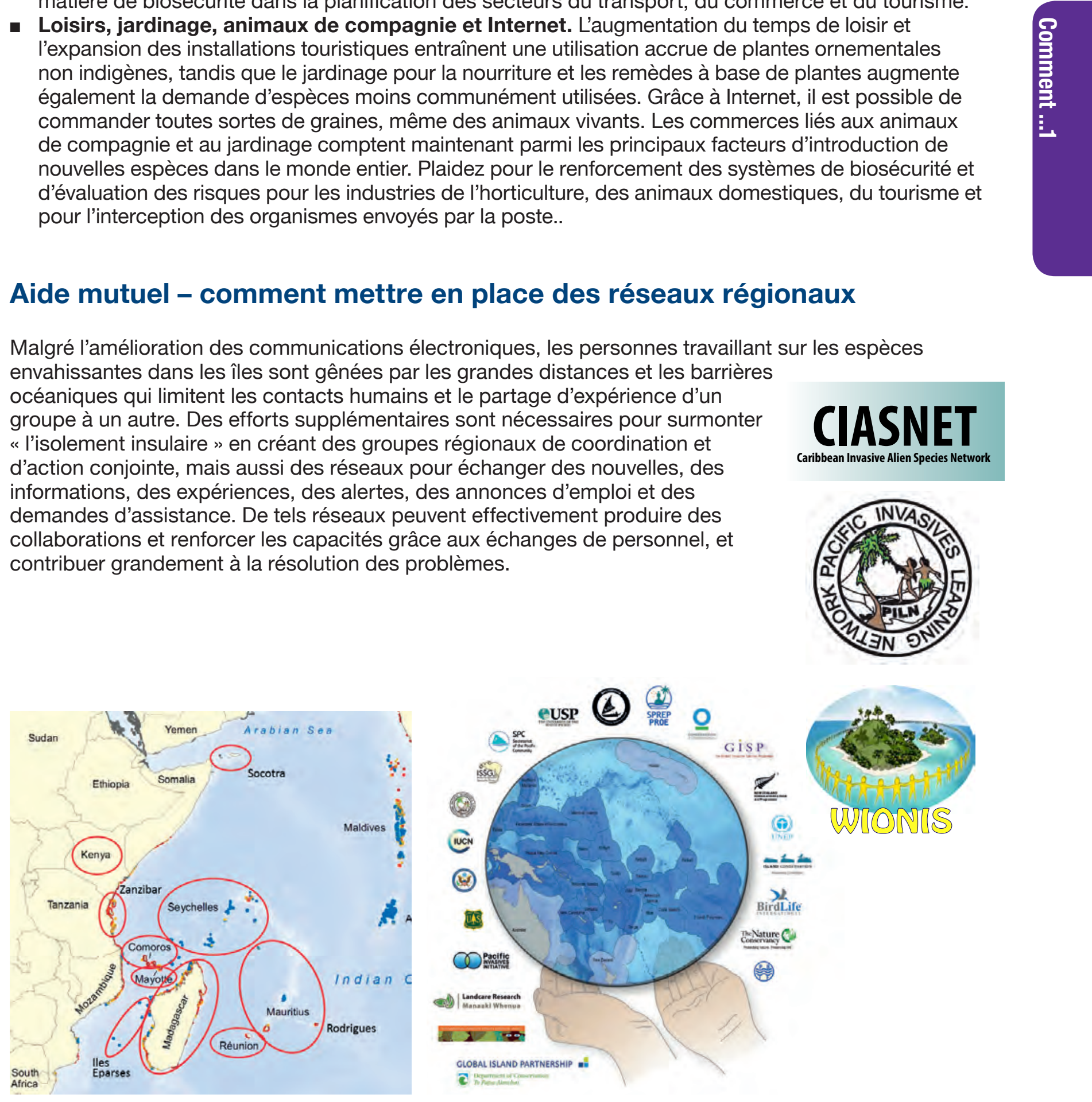


\section{L'exemple du Pacifique}

Le Pacifique (Océanie) montre un bon exemple de coordination régionale, hautement inclusive et efficace, en matière d'espèces envahissantes, et soutenue par un solide cadre institutionnel régional comprenant des organisations de services régionaux, un réseau de professionnels, un partenariat d'organisations qui s'attaque aux problèmes liés aux espèces envahissantes et deux listes de diffusion d'emails.

Deux organisations intergouvernementales régionales aident leurs pays et territoires membres à gérer l'environnement de l'Océanie et ce qui le menace. Le Programme régional océanien de l'environnement (PROE) soutient la protection et l'amélioration du milieu naturel, tandis que la Communauté du Pacifique (CPS) encourage le développement économique durable en soutenant les secteurs de l'agriculture, de la foresterie, de la pêche et de la santé. Les deux ont des programmes liés aux espèces envahissantes.

La conservation de la nature est coordonnée dans la région par un réseau d'organisations non gouvernementales, de donateurs et autres, connu sous le nom de Table ronde des îles du Pacifique pour la conservation de la nature (PIRT) ; il sert de forum pour la collaboration et a l'approbation officielle des gouvernements insulaires du Pacifique. Le PIRT mène ses activités par le biais de groupes de travail, et le groupe de travail sur les espèces envahissantes du PIRT est connu sous le nom de Partenariat océanien sur les espèces envahissantes (PIP). Le PIP comprend 40 organisations régionales et internationales travaillant sur différents aspects des espèces envahissantes dans le Pacifique, et le PIP coordonne la planification et l'assistance de ces organisations aux îles du Pacifique, pour une gestion plus efficace des espèces envahissantes. Le PIP se réunit chaque année et ses membres élaborent un plan d'action annuel conjoint. Le PIP est coordonné par le PROE, tandis que la présidence du PIP est assurée par les organisations membres.

Le Réseau d'apprentissage sur les invasions du Pacifique (PILN) est un réseau de professionnels qui s'articule autour d'équipes insulaires ou nationaux comprenant des représentants d'organisations gouvernementales et non gouvernementales. L'adhésion au PILN est également ouverte aux individus. Comme le PIP, le PILN est géré par un coordinateur hébergé par le PROE. Les membres du PILN utilisent le réseau pour partager des compétences, des connaissances et des nouvelles, mais aussi pour formuler des demandes d'aide. Le PILN est le réseau des praticiens nationaux, tandis que le PIP est le partenariat des organisations qui souhaitent les aider. Le PILN est membre du PIP afin de faciliter les liens entre les "utilisateurs » et les « fournisseurs » de services en matière d'espèces envahissantes.

L'Initiative du Pacifique sur les espèces envahissantes (PII) est une petite équipe pluridisciplinaire de spécialistes qui travaille au renforcement de la capacité des îles du Pacifique pour la gestion efficace des espèces envahissantes, en s'appuyant notamment sur les experts externes néozélandais et autres. La Pll est également membre du PIP.

Le PROE et le PIP ont tous deux des membres appartenant la catégorie des « pays développés » et ayant des intérêts dans la région : Australie, France, Nouvelle-Zélande, Royaume-Uni et États-Unis. Ces derniers contribuent à la gestion des espèces envahissantes grâce à leurs programmes d'aide et de développement, notamment en finançant le PROE et d'autres initiatives régionales et en fournissant des services d'experts, de formation et de recherche.

Le Pacifique est de loin le plus grand océan de la planète, avec plus d'îles que partout ailleurs. La coordination sous-régionale est donc également utile, en particulier au niveau des politiques. À cet égard, des Conseils régionaux sur les espèces envahissantes en Micronésie et en Mélanésie ont été créés pour la planification et la coordination entre les îles de ces régions. Ils donnent des conseils sur les espèces envahissantes aux plus hauts niveaux du gouvernement dans leurs îles, y compris au niveau des chefs d'État.

Tout cela peut sembler complexe, mais ça marche! Et avec tant de personnes impliquées, il y a toujours quelqu'un pour maintenir l'engouement pour une collaboration efficace.

Les Directives pour la gestion des espèces envahissantes dans le Pacifique (PROE 2010) ont servi de cadre d'action pour tous les pays et territoires d'Océanie et pour tous les réseaux et organisations susmentionnés, y compris les organisations membres du PIP. Vous pouvez utiliser les Directives mondiales que vous lisez maintenant de la même manière. 


\section{Comment créer puis étendre votre réseau : du local au régional}

En plus de leur rôle dans la coordination entre les îles, les réseaux fournissent également une plate-forme inestimable permettant aux différents secteurs de se parler et de coordonner le travail au sein d'une même île ou d'un même pays. Il y a souvent une barrière entre les différents secteurs : l'environnement, l'agriculture, la foresterie ou même les services phytosanitaires. Les premières mesures du PILN ont été d'encourager la formation d'équipes nationales qui ont réuni tous ces secteurs autour d'une même table, leur permettant de découvrir leurs intérêts communs et de comprendre les besoins de chacun. L'inclusion du secteur des douanes et des quarantaines dans ces réseaux est essentielle, car elles doivent passer de leur rôle traditionnel axé sur la santé des plantes et des animaux et sur les parasites agricoles, pour couvrir des espèces non indigènes préoccupantes pour l'environnement. Ensuite, le regroupement des équipes PILN des différentes îles (approche ascendante participative, chez PILN) et le regroupement des principales organisations régionales (approche descendante du sommet vers la base, chez PIP) ont été les éléments cruciaux dans la formation du cadre régional décrit ci-dessus. Ainsi, former un réseau régional peut être mise en pratique très efficacement en commençant sur le plan national, en parlant, en consultant et en encourageant les comités locaux sur les espèces envahissantes ou des groupes similaires à se former, puis en établissant des liens entre les îles.

\section{Deux éléments essentiels : de vraies réunions et un coordinateur enthousiaste}

Reconnaître que des leçons peuvent être apprises des voisins et mettre en place des actions conjointes avec eux jouent un rôle important dans la création et le renforcement du réseau, tout comme le fait de développer des amitiés lors de réunions en face à face. De telles réunions semblent essentielles, même en ces temps de médias sociaux et d'Internet : apprendre à connaître les gens personnellement est la meilleure façon de briser la glace, de ne plus avoir peur de demander de l'aide et d'organiser des échanges. Un coordinateur de réseau enthousiaste semble également indispensable : quelqu'un qui peut constamment promouvoir et encourager. Peu importe combien on essaie de « responsabiliser les membres ", le mieux est d'engager une personne (pas nécessairement à plein temps) qui enverra continuellement des messages par la liste de diffusion, mettra en place des réunions et maintiendra généralement en activité le réseautage. Le coordinateur du PILN était initialement sous contrats temporaires jusqu'à ce qu'il ait un poste permanent dans une organisation régionale, et cette transition semble essentielle. Les réseaux avec un coordinateur actif restent en activité, mais sans quelqu'un d'enthousiaste et dévoué dans ce rôle, ils stagnent. Cependant, mis à part le besoin d'un coordinateur, avec peut-être un petit budget pour les voyages, la maintenance du réseau ne nécessite pas beaucoup d'argent.

\section{À quoi ressemble un réseau qui fonctionne bien ?}

- Un coordinateur de réseau (pas nécessairement à plein temps) avec ce rôle dans le cadre de son travail (idéalement employé par une organisation régionale).

- Des communications électroniques fréquentes et pertinentes et des réunions occasionnelles du réseau.

- Des équipes ou des comités multisectoriels nationaux fiables sur les espèces envahissantes se rencontrant régulièrement, car ils voient la valeur de ces échanges.

- Un groupe de planification conjoint régional pour l'identification des priorités conjointes et des actions de collaboration.

- Des liens professionnels entre les professionnels de différentes îles, qui se transforment en solides amitiés.

\section{Dans quelle mesure les autres régions océaniques ont-elles atteint cet objectif ?}

Le Réseau de l'océan Indien occidental sur les espèces envahissantes (WIONIS) a été inspiré par l'expérience du Pacifique, mais a commencé d'une manière fondamentalement différente. La décision a été prise de lancer le réseau lors d'une réunion régionale en 2012, et WIONIS a commencé sur le plan régional, sans former au préalable une base solide d'équipes nationales. Le réseau a été soutenu par un coordinateur employé pour un projet régional sur les espèces envahissantes, financé seulement pendant ses premiers six ans. WIONIS a développé une liste d'emails plus active que le PILN, mais toujours avec de nombreuses contributions identifiées et diffusées par le coordinateur. Certaines îles de l'OIO ont des comités multisectoriels sur les espèces envahissantes, mais ceux-ci n'ont pas d'engagements institutionnels vis-à-vis du réseau régional et, par conséquent, il manque l'élément clé de la priorisation et de la planification conjointes interinsulaires. En comparaison avec le Pacifique, les liens entre les comités insulaires sont moins efficaces, avec moins de réunions régionales sur les espèces envahissantes et peu d'actions conjointes. 
Les initiatives de coopération sur les espèces envahissantes dans les Caraïbes ne sont pas non plus axées sur une action conjointe. Les pays membres de la CARICOM (Communauté des Caraïbes) ont plusieurs organes de coordination, notamment l'Agence caribéenne de santé agricole et de sécurité sanitaire des aliments, le Forum des directeurs phytosanitaires des Caraïbes (CPHD), l'Initiative de sauvegarde de la Grande Caraïbe et le Groupe de travail sur les espèces envahissantes des Caraïbes (CISWG), qui mettent l'accent principalement sur les systèmes de santé animale et végétale et de sécurité alimentaire dans le contexte de la facilitation des échanges, et le CISWG est resté inactif pendant quelques années. D'autres initiatives régionales mènent certaines actions, telles que CAR-SPAW-RAC (voir Ressources p. 41). Le Réseau sur les espèces exotiques envahissantes des Caraïbes (CIASNET) couvre toutes les îles et tous les aspects des espèces envahissantes dans les Caraïbes ou les menaçant, par sa liste de diffusion d'emails carib_ias_threat. Mais pour atteindre la force et l'influence dont bénéficient le PIP et le PILN dans le Pacifique, le CISWG doit être revitalisé, tandis que le CIASNET et le CPHD doivent renforcer les liens avec les groupes les plus puissants qui gèrent la sécurité agricole et alimentaire. Une initiative ou un projet régional d'envergure pourrait y parvenir, mais là aussi il faut un coordinateur permanent.

L'Initiative sur les espèces exotiques envahissantes dans les territoires d'outre-mer (TOM) français a été lancée en 2005 par le Comité français de l'UICN (voir Ressources, p. 41) avec un responsable de projet à plein temps et des coordinateurs locaux dans chaque territoire (experts reconnus ou gestionnaires de ressources naturelles). Ses premiers résultats comprenaient un inventaire des espèces envahissantes et leurs impacts dans tous les TOM, des ateliers régionaux dans les Caraïbes, le Pacifique et l'océan Indien, et la production de directives techniques et de brochures d'information pour les praticiens. Cette initiative recoupe et complète le réseautage régional dans chaque région océanique et bénéficie du soutien national (français) plutôt que de dépendre d'une organisation régionale.

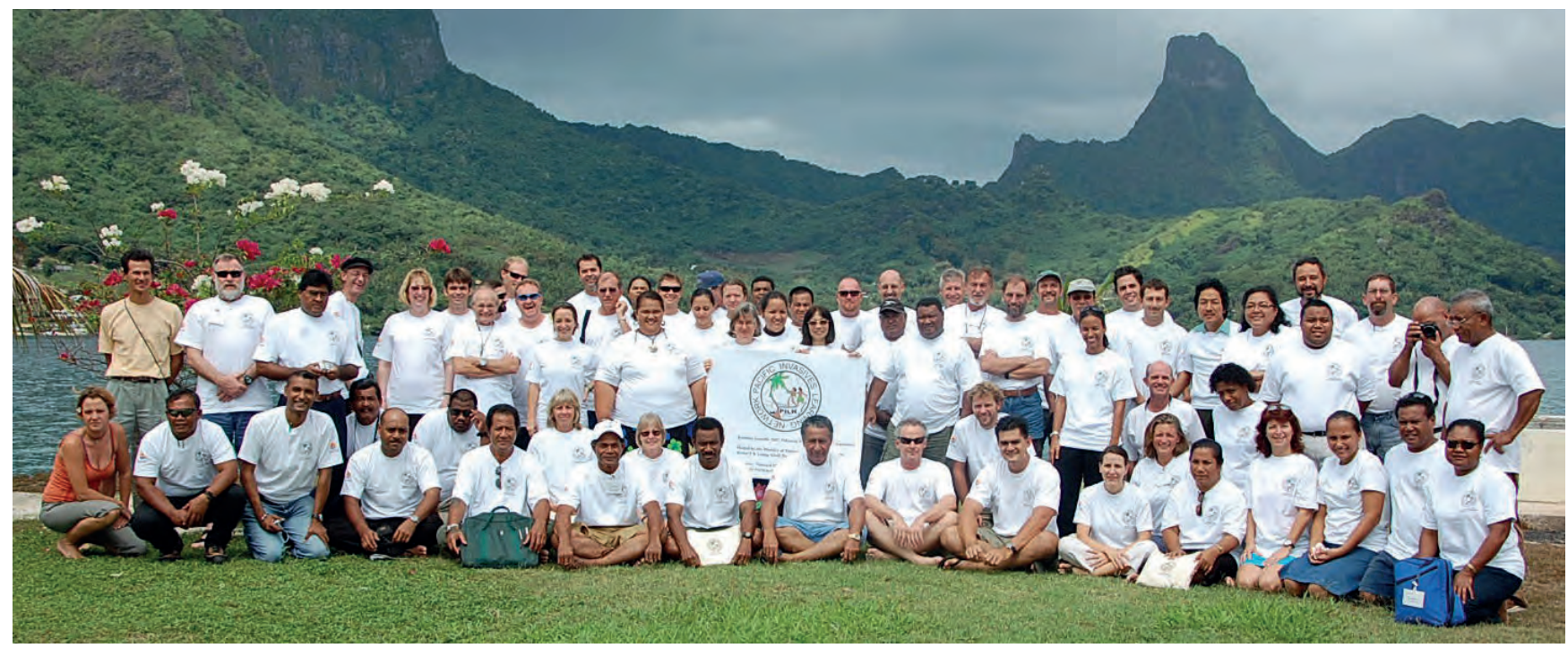

A nouveau une réunion de réseau réussie... 


\section{Comment : rédiger le plan, prioriser, prendre des décisions}

Cette partie concerne principalement la planification programmatique : rédiger et utiliser des stratégies, des politiques et des plans d'action pour guider les organisations responsables de la prévention et de la gestion des espèces envahissantes. Mais la première étape consiste à déterminer qui rédige la stratégie ? Un département ou une organisation travaillant en solo peut planifier ses propres programmes, mais une stratégie nationale, régionale ou insulaire doit être menée par un effort de planification multi-institutionnelle. Par exemple, une stratégie et un plan d'action nationaux contre les espèces envahissantes sont idéalement mis en place par une sorte de comité national sur les espèces envahissantes (CNEE), tandis que des groupes similaires à plus grande échelle peuvent rédiger des plans régionaux ou à plus petite échelle des plans pour chaque île.

\section{Comment constituer et faire fonctionner un Comité national sur les espèces envahissantes}

Vous pouvez commencer par n'importe quelle équipe préexistante ou par un groupe informel de collègues dans différentes organisations. Quand on se réfère à l'expérience du Pacifique décrite sur pp. 22-23, l'équipe du PILN ( $y$ compris les ministères, les ONG et autres) est souvent devenue le CNEE, et les équipes qui étaient plus fortes au niveau technique se sont rapidement intégrés aux processus nationaux de planification, influençant donc les décideurs, prouvant que l'approche ascendante participative fonctionne.

Un CNEE prospère a besoin de son propre coordinateur, de préférence une personne au niveau opérationnel, qui s'occupe des espèces envahissantes au quotidien dans son travail et qui peut diriger activement le processus de planification. Le président du comité peut être une personne de plus haut niveau, comme un chef de département, pour donner au comité de la crédibilité et de l'influence auprès du gouvernement, mais idéalement, le président devrait aussi être passionné par la gestion des espèces envahissantes. Lorsque le leadership est faible, le comité est faible - les champions jouent un grand rôle, y compris lors de la création du comité, et plus il y en a, mieux c'est. Ils peuvent inclure le coordinateur et le président du CNEE, mais la participation d'autres personnes actives et enthousiastes, y compris des ONG et du secteur privé, contribue grandement à encourager et à promouvoir les idées. Une sorte de charte du comité peut aider à clarifier le but, les rôles et les procédures. Les bons comités comptent parmi leurs membres des personnes qui peuvent influencer leurs propres organisations (et toucher les autres organisations) pour entraîner des changements et s'assurer que la gestion des espèces envahissantes est bien intégrée dans la planification et la budgétisation du département, ensuite intégrée dans la planification et la budgétisation nationales. Lorsque le CNEE est incorporé dans le système gouvernemental, il se peut qu'il reçoive des ressources gouvernementales pour les opérations et se fasse mieux entendre, et il peut alors assumer un rôle de leadership national pour la gestion des espèces envahissantes.

\section{Gardez-le actif, pertinent, intéressant et utile}

Pour éviter l'apathie, les comités doivent éviter de se cantonner à un format répétitif et de se réunir de façon routinière, surtout s'il n'y a pas grand-chose à dire. Veillez à vous réunir quand il y a matière à discussion, mais aussi à intervalles réguliers (quelques fois par an), même si rien n'est à l'ordre du jour, car le simple fait d'être dans la même pièce incitera souvent les personnes à saisir l'occasion de faire ressortir des problèmes. Pour cette raison, les réunions doivent être aussi amicales et accueillantes que possible, avec une atmosphère égalitaire et détendue, et un président sympathique qui encourage tout le monde à contribuer.

\section{Comment rédiger une stratégie et un plan d'action sur les espèces envahissantes (SPAEE)}

\section{Pourquoi rédiger une SPAEE ?}

Parce qu'il n'y a jamais assez de ressources pour tout faire. Une SPAEE peut :

- rapprocher les différents secteurs et la communauté élargie pour convenir des priorités,

- montrer que vous avez parlé, que vous avez réfléchi stratégiquement et que vous êtes prêt à agir,

- porter les problèmes liés aux espèces envahissantes à l'attention des décideurs et des organismes de financement,

- fournir des arguments solides pour les persuader de financer des actions prioritaires approuvées par tous. 


\section{Privilégiez l'inclusion}

Un CNEE est le groupe idéal pour diriger la rédaction d'une SPAEE national. L'adoption et l'extension d'un processus de planification existant (tel que le processus SPANB) peuvent aider à faire avancer un CNEE et un plan, car (une partie de) l'équipe de planification sera déjà là. Mais assurez-vous que la représentation est élargie pour inclure tous les principaux secteurs ou organisations manquants. II est essentiel d'inclure tous les secteurs impliqués dans l'utilisation ou la gestion des espèces envahissantes, y compris les organisations gouvernementales, les ONG et les organisations de recherche travaillant dans l'environnement, l'agriculture, I'horticulture, la foresterie, l'aquaculture, la quarantaine, les services de gestion des mauvaises herbes et des ravageurs, la santé, le transport, le tourisme, l'application de la loi... - en inclure autant que possible. Beaucoup d'entre eux seront sur le CNEE, mais vous devrez peut-être inviter des secteurs connexes tels que l'aménagement du territoire, le changement climatique et le développement économique, et si vous pouvez intéresser le ministère des Finances ou le Premier ministre, tant mieux. Idéalement, une SPAEE nationale devrait être liée à la SPANB ou à la stratégie nationale de développement du pays et devrait être approuvée par le gouvernement. Qu'elle soit nationale, mono-insulaire ou sectorielle, la SPAEE doit être préparée de façon inclusive, de sorte que tous ceux qui sont responsables des espèces envahissantes la considèrent comme « leur » travail et « leur » plan.

\section{Apprenez d'un expert et utilisez ces Directives pour vous aider}

Une des principales fonctions de ces Directives est d'orienter toutes les étapes de la création d'une SPAEE. Utilisez-les pour vous assurer de ne rien oublier. Les meilleures SPAEE sont préparées après discussions entre les parties prenantes, animées par un expert en matière d'espèces envahissantes et en planification (pas seulement en planification). Une telle personne aide beaucoup en apportant des conseils d'experts ainsi qu'en facilitant et compilant les idées. L'aide d'un expert permet aussi de repérer des lacunes. C'est donc une bonne idée de faire réviser les ébauches de stratégies et de plans d'actions par un expert ou par un groupe d'experts externe.

\section{Contenu et organisation}

Qu'il s'agisse de planifier pour une île, une nation insulaire ou même une organisation individuelle, mettez l'accent sur ces trois parties principales du plan : Introduction, Stratégie et Plan d'action.

L'Introduction à un bon plan consiste en une « analyse de la situation » qui décrit ce qui est connu et inconnu des espèces envahissantes dans les îles concernées, leurs impacts sur les zones naturelles, les espèces menacées et endémiques, les moyens de subsistance, la santé et l'économie, leurs impacts potentiels futurs si les espèces envahissantes ne sont pas gérées, les im-pacts probables des espèces qui ne sont pas encore arrivées dans les îles, mais qui pourraient faci-lement être introduites, et les voies d'introduction. Elle passe également en revue les mesures de gestion passées et présentes, identifie les principales lacunes du programme et les problèmes qui compliquent la gestion (manque de politique, de législation, d'organisations responsables, d'informations, d'expérience, d'experts, de fonds, de relations avec les donateurs ou d'assistance technique). Enfin, elle identifie les organisations à impliquer dans la gestion (mise en œuvre du plan), leurs rôles, responsabilités et capacités, et les lois, mandats et ressources financières dispo-nibles pouvant aider.

Quant à la Stratégie, elle peut être basée sur des buts généraux, destinés à résoudre les problèmes et les lacunes en termes de connaissances et d'actions identifiés dans l'Introduction. Chaque but peut être abordé par une série d'objectifs plus détaillés. La stratégie devrait également nommer qui est responsable (souvent le CNEE) de diriger sa mise en œuvre, de faire le suivi des progrès réalisés, d'évaluer les succès atteints, de rendre compte aux parties prenantes des progrès ou du manque de progrès, et d'assurer la continuation et le suivi du plan à la fin de sa période de mise en œuvre.

Le Plan d'action est la partie la plus importante d'une SPAEE efficace et présente les prio-rités spécifiques retenues pour actions pendant la durée de la SPAEE. Chaque action devrait con-tribuer à atteindre un ou plusieurs objectifs de la stratégie, être précise et spécifique, mais aussi indiquer : qui en est responsable (organisme gouvernemental, ONG, groupe communautaire... n'incorporez une action que si l'organisation désignée est d'accord !), quand elle sera réalisée (une période déterminée), quel en sera le coût et d'où proviendra le financement. L'état d'avancement des actions devrait être suivi, afin d'en tenir compte lors des plans d'action ultérieurs. La SPAEE devrait inclure des actions d'évaluation et, vers la fin de la durée de vie de la SPAEE, de planification ultérieure. 


\section{Principes pour rédiger une SPAEE}

- Prendre en considération les dix Domaines thématiques de la Liste de contrôle de ces Directives

- Privilégier l'inclusion - le processus doit être pleinement participatif
- Inclure les secteurs et les agences clés

- Associer à la SPANB et/ou à la Stratégie nationale de développement

- Planifier pour l'avenir - que se passe-t-il lorsque cette SPAEE se termine?

\section{Un modèle pour une SPAEE}

PARTIE 1: INTRODUCTION avec « analyse de situation »

Cette partie couvre le contexte, les mesures prises, les mesures nécessaires et les domaines nécessitant une attention particulière.

- Résumez ce que l'on sait des espèces introduites et envahissantes dans vos îles (ajoutez une annexe avec des listes d'espèces, des indications sur l'impact pour chacune d'entre elles). Les listes sont-elles assez complètes ou vraiment incomplètes ?

- Résumez ce que l'on sait (i) des impacts actuels (sur les zones naturelles, les espèces menacées et endémiques, les moyens de subsistance, la santé et l'économie), (ii) des impacts futurs potentiels (si les espèces envahissantes ne sont pas gérées),

(iii) des impacts probables d'espèces pas encore présentes dans les îles, mais qui pourraient être introduites facilement, (iv) des voies d'invasion. Quelle connaissance manque?

- Passez en revue la gestion passée et présente des espèces envahissantes. Quelle action manque, et pourquoi manque-t-elle ? Utilisez la Liste de contrôle de ces Directives pour examiner les programmes actuels et identifier les lacunes.

- Identifiez quelles institutions devraient être impliquées, quelle est leur capacité (y compris leurs budgets) et quelles politiques, lois et réglementations sont en place. Que manque-t-il ?

\section{PARTIE 2: STRATÉGIE - définir les Buts et les Objectifs}

- Les buts représentent les aboutissements - que faut-il pour combler les lacunes et résoudre les problèmes identifiés ci-dessus?

- Les objectifs représentent ce que vous voulez arriver pour atteindre votre but.

\section{Un exemple d'un But avec ses Objectifs peut être :}

But 1 : Le taux d'arrivée de nouvelles espèces dans nos îles est réduit.

Objectif 1.1 La Direction de la quarantaine applique une évaluation des risques à toutes les nouvelles introductions proposées et établit des listes d'organismes et de produits autorisés et interdits.

Objectif 1.2. Le personnel de la quarantaine et des douanes inspecte $10 \%$ de tous les conteneurs et $100 \%$ des bagages des passagers pour intercepter des organismes et les éliminer en toute sécurité.

\section{PARTIE 3 : PLAN D’ACTION - définir des Actions pour atteindre les Objectifs}

- Pour chaque Objectif de la stratégie, identifiez une ou plusieurs Actions.

- Chaque action doit être «SMART » (Spécifique, Mesurable, Assignée à quelqu'un, dotée de Ressources et limitée dans le Temps).

- Si vous n'avez pas déjà l'assistance ou le financement nécessaire, décrivez l'Action comme ceci : « Demander de l'aide/ du financement de $X Y Z$ pour faire ABC... ».

\section{Des exemples d'Objectifs et de leurs Actions pourraient être :}

Objectif 1.1 La Direction de la quarantaine applique une évaluation des risques à toutes les nouvelles introductions proposées.

\begin{tabular}{llllll} 
& Action & Responsable & Complétée d'ici & Coût & Source \\
\hline 1.1a & $\begin{array}{l}\text { Former le personnel de quarantaines } \\
\text { à l'évaluation des risques }\end{array}$ & Direction de la quarantaine & Mai 2020 & $10000 \$$ & Assistance du pays voisin X \\
\hline 1.1b & $\begin{array}{l}\text { Inclure des procédures d'évaluation } \\
\text { des risques dans la législation sur la }\end{array}$ & Ministère de l'Agriculture & Août 2020 & - & $\begin{array}{l}\text { Dans les limites du budget du } \\
\text { gouvernement }\end{array}$
\end{tabular}
biosécurité

Objectif 1.2 Le personnel chargé de la quarantaine et des douanes inspecte $10 \%$ de tous les conteneurs et $100 \%$ des bagages des passagers pour intercepter des organismes et les éliminer en toute sécurité.

\begin{tabular}{llllll} 
& Action & Responsable & Complétée d'ici & Coût & Source \\
\hline 1.2a & $\begin{array}{l}\text { Installer une machine à rayons X à l'aéroport } \\
\text { principal }\end{array}$ & Quarantaine et douanes & Mars 2021 & 10000 \$ & Projet X financé par bailleur Y \\
\hline 1.2b & $\begin{array}{l}\text { Augmenter le taux d'inspection des conteneurs à } \\
10 \% \text { en recrutant 2 nouveaux employés }\end{array}$ & $\begin{array}{l}\text { Quarantaine et douanes } \\
\text { (1 nouvel employé par service) }\end{array}$ & Juillet 2020 & $\begin{array}{l}30000 \text { \$ Budget du gouvernement } \\
\text { par année }\end{array}$
\end{tabular}


La SPAEE devrait considérer les dix Domaines thématiques de la Checklist des Directives (pp. 1-14), y compris établir les fondations nécessaires pour agir, comprendre les espèces envahissantes, prioriser les activités, empêcher les introductions, gérer les espèces envahissantes établies et restaurer la biodiversité et d'autres valeurs affectées.

\section{Planifiez pour réussir!}

Les gens sont inspirées par les victoires, même les plus petites, et donc les professionnels impliqués ressentiront que cela valait la peine de faire partie de leur équipe, leur CNEE et leur réseau. Pour cette raison, le choix d'objectifs-cibles réalistes et atteignables est un élément essentiel d'une bonne planification (voir pp. 31-38). Plus important encore, pensez au-delà du plan - ce qui importe c'est l'action. Les espèces envahissantes représentent souvent une priorité mineure pour les gouvernements insulaires, qui peuvent parler du problème et faire des plans d'action, mais ne pas forcément fournir assez d'argent pour les mettre en œuvre. Sur le papier, les pays et territoires insulaires ont souvent des stratégies et des plans d'actions très attrayants, mais concrètement sur le terrain ils ne font pas beaucoup de gestion des espèces envahissantes parce que la plupart des plans ne bénéficient pas du soutien financier nécessaire ou d'un engagement réel des parties prenantes.

Pour surmonter cela, il est essentiel d'être réaliste et pratique. Visez à faire en sorte que les actions puissent être accomplies, plutôt que d'essayer d'être trop ambitieux. Un slogan utile est de " transformer les mots en action »- pas facile, mais c'est le but. La planification stratégique peut aboutir à une longue liste de souhaits impossible, mais une planification réussie et inspirante se concentre sur ce qui peut réellement être fait, en premier lieu avec les ressources existantes, pour ensuite progresser vers des objectifs plus ambitieux nécessitant des fonds supplémentaires. Commencez petit, par des objectifs-cibles faciles, et assurez-vous d'en atteindre certains. Par exemple, il est facile de décider qu'une plante envahissante répandue comme la vigne Merremia (Pacifique) ou le goyavier de Chine Psidium cattleianum (océan Indien), ou un ravageur commun introduit comme les mangoustes ou les mainates sont des priorités, car ce sont des " problèmes évidents ". Mais essayer d'y remédier est décourageant et n'aboutit à rien lorsque les ressources sont insuffisantes. Mieux vaut sélectionner des cas d'infestation précoce où une éradication complète de l'espèce est possible, améliorer en même temps votre système de biosécurité insulaire et passer progressivement à des problèmes plus ardus.

Ensuite, vous pouvez célébrer les succès, même les plus petits, et vous en servir pour obtenir du soutien, en démontrant comment cela peut être répliqué, à plus grande échelle et mieux, et comment les autres peuvent faire la même chose. A cet égard, les échanges et les ateliers sont d'une grande aide, et ils s'étendent au-delà du contexte local jusqu'au niveau régional et plus encore. La communication est très importante, même en cas d'échecs. Un bulletin d'information ou un site Web peut y être un bon outil, en commençant en toute simplicité. Le coordinateur doit immanquablement le maintenir et encourager les contributions.

\section{Modèles de SPAEE}

Les SPAEE existent pour de nombreuses îles, beaucoup d'entre elles reposant sur les recommandations de la Convention sur la diversité biologique, comme les objectifs d'Aichi (voir Ressources p. 40). Vous pouvez trouver beaucoup de bonnes SPAEE en ligne et les utiliser comme modèles, alors que vous pouvez trouver des conseils supplémentaires sur la rédaction d'une SPAEE dans la Battler Resource Base du Pacifique (voir Ressources p. 40).

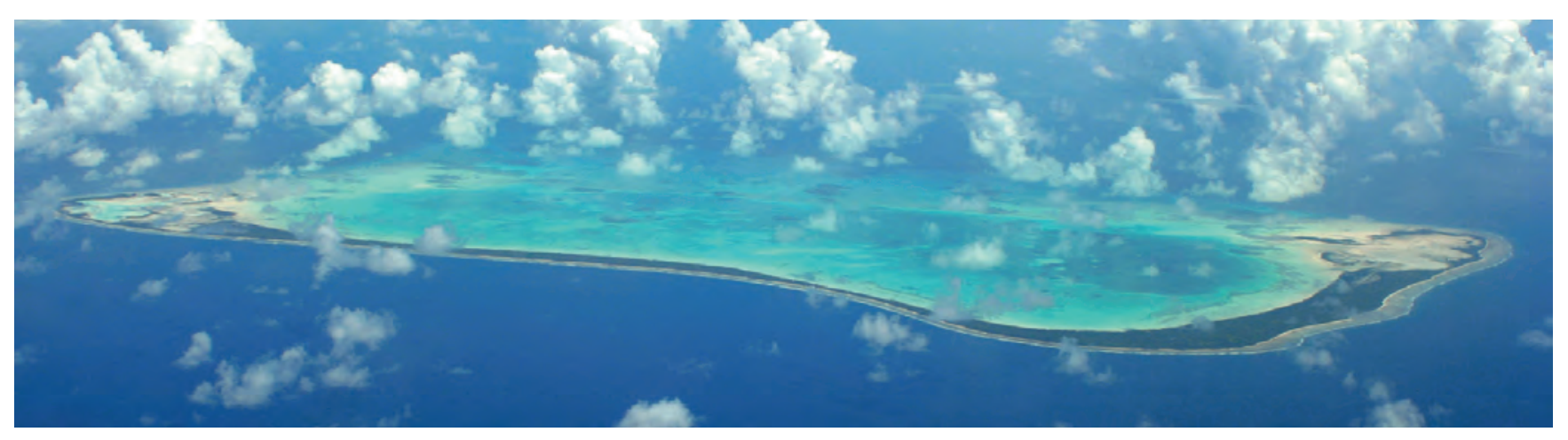

Atoll Maiana, Kiribati. 


\section{Comment prioriser}

La hiérarchisation des priorités fait partie de la prise de décision tout au long du processus de planification, mais se produit le plus clairement à deux étapes principales :

- Prioriser les domaines d'action. Ces décisions de planification de plus haut niveau sont prises en compte dans la partie A1 de la Checklist de ces Directives.

- Prioriser les espèces, les voies d'introduction et les sites. Ces décisions de planification opérationnelle sont examinées dans la partie B2 de la Checklist de ces Directives.

\section{Prioriser les domaines d'action}

La principale recommandation ici est d'utiliser un aide-mémoire, pour s'assurer que rien d'important n'est négligé. C'est précisément la fonction de la Checklist de ces Directives ci-dessus (p. 1-14). Voir le Domaine thématique A1 de la Checklist pour des conseils et des principes généraux, et utilisez la Checklist dans son ensemble pour choisir les domaines importants pour votre pays, votre île ou votre organisation. Utilisez-la également pour identifier les lacunes et les domaines négligés dans votre programme actuel en matière d'espèces envahissantes.

La Checklist vous aidera à prendre en compte, à inclure ou à exclure de votre plan chaque domaine d'action particulier. Par exemple, votre île pourrait être plus préoccupée à l'heure actuelle par la biosécurité (pour bloquer l'entrée de certaines espèces) que par, disons, le contrôle biologique par exemple, ou si vous planifiez pour une organisation, vous serez évidemment plus préoccupé par les mandats officiels de votre organisation ou par le domaine d'expertise technique de votre ONG. Si vous rédigez une SPAEE, vous devrez examiner attentivement ce que vous allez omettre (pour l'instant), car même si, idéalement, vous devez couvrir tout, il est essentiel d'être réaliste et de ne planifier que les projets que vous pensez pouvoir financer ou pour lesquels vous pouvez trouver des fonds. Vous pouvez utiliser la Checklist pour vous aider à établir des priorités à court et à long terme, même si vous n'avez pas encore assez de fonds pour les priorités à plus long terme.

\section{Que faisons-nous bien et que faisons-nous moins bien ?}

Un certain nombre d' études d'analyse des lacunes (p. ex., Doherty et Boudjelas 2010 pour le Pacifique ; Key 2017 pour les territoires britanniques d'outre-mer ; Boudjelas 2018 pour l'océan Indien occidental), ainsi que des consultations informelles dans le cadre de l'élaboration de ces Directives montrent de manière générale à quel point les différents domaines d'action qui composent les dix Domaines thématiques de la Checklist de ces Directives sont bien ou mal couverts par les SPAEE, des plans similaires ou des programmes insulaires sur les espèces envahissantes. Presque uniformément à travers les îles et les régions océaniques du monde entier, la couverture de la meilleure (vert) à la pire (rouge) suit cet ordre :

\begin{tabular}{|c|c|c|c|c|c|c|c|c|c|}
\hline C2 & A2 & A3 & B3 & A4 & C1 & B2 & B1 & A1 & C3 \\
\hline Gestion & $\begin{array}{c}\text { Générer du } \\
\text { soutien }\end{array}$ & $\begin{array}{c}\text { Renforcement } \\
\text { des capacités }\end{array}$ & Recherche & $\begin{array}{c}\text { Législation, } \\
\text { politique }\end{array}$ & Biosécurité & Priorisation & $\begin{array}{c}\text { Base de } \\
\text { référence, } \\
\text { suivi }\end{array}$ & Planification & Restauration \\
\hline 1 & $\mathbf{2}$ & $\mathbf{3}$ & $\mathbf{4}$ & $\mathbf{5}$ & $\mathbf{6}$ & $\mathbf{7}$ & $\mathbf{8}$ & $\mathbf{9}$ & $\mathbf{1 0}$ \\
\hline
\end{tabular}

\section{Domaines communément négligés - ne les oubliez pas !}

Vous trouverez quelques leçons flagrantes dans le tableau ci-dessus.

- Même si la Gestion des espèces envahissantes établies (Domaine thématique C2), Générer du soutien (A2) et le Renforcement des capacités (A3) sont généralement couverts par la plupart des plans ou des programmes sur les espèces envahissantes, la Biosécurité (C1), qui est la manière la plus rentable et la plus sûre de gérer un grand nombre d'espèces envahissantes (en les empêchant d'entrer), est généralement mal couverte, dans la plupart des îles du monde. Elle est généralement sousfinancée, souvent orientée uniquement contre les parasites agricoles, et parfois complètement négligée. Lors de la planification, prenez en compte les modèles de prédictions (Action B1.3a : quelles espèces arriveront probablement et de quelle façon), les systèmes formels de quarantaine (contrôle aux frontières), ainsi que la sensibilisation et la biosécurité communautaire informelle pour protéger les petites îles. 
- La Recherche (B3) et la Législation, la politique et les protocoles (A4) sont généralement pris en compte d'une manière ou d'une autre, mais pas toujours comme il se doit. Et vous, en faites-vous vraiment assez ? La « recherche » peut être considérée comme une excuse pour ne pas agir, mais une recherche correctement appliquée est essentielle pour obtenir une bonne gestion.

- La Priorisation (B2), les Bases de référence et le suivi (B1) et la Planification (A1) sont omis dans la plupart des plans et des programmes. Ces trois domaines sont à la base de la prise de décisions et de la planification, ce qui suggère fortement que :

- les décisions reposent principalement sur des informations lacunaires (données de base insuffisantes),

- elles sont le plus souvent prises arbitrairement ou subjectivement, sans sélection objective des priorités ou sans suivre le principe de base de choisir avec soin les activités que vous pouvez réaliser, - souvent, nous ne savons pas si les projets ont eu un effet ou pas (pas de suivi).

L'aspect le plus inquiétant de la négligence dans ces domaines c'est que la plupart des plans ne sont pas très bien conçus et que la plupart des programmes qui en résultent sur les espèces envahissantes sont déséquilibrés. Une des raisons principales de produire ces Directives est d'ailleurs ce manque de bonne planification. Essayez de vous assurer que votre planification est ellemême correctement planifiée (A1) et priorisée (B2) - utilisez la Checklist de ces Directives, et faites de la priorisation appropriée une action dans votre plan.

- La Restauration (C3) est également généralement omise. Elle est étroitement liée au suivi (B1) parce que, sans suivi, nous ne savons pas quelle restauration post-gestion peut être nécessaire. Le suivi (B1) et la restauration (C3) sont essentiels - essayez de vous assurer que les deux soient inclus et financés.

De plus, dans les Domaines thématiques individuels, les éléments suivants sont souvent omis : - La réaction rapide aux incursions (partie du domaine thématique C1 Biosécurité) et l'éradication (partie du C2 Gestion) sont à long terme les méthodes de gestion les moins chères (après la prévention), mais elles sont sous-utilisées principalement parce que les gouvernements et les personnes ordinaires ne voient pas la valeur de la gestion d'une espèce avant qu'elle ne devienne un problème, alors qu'il est plus facile et moins cher de l'éradiquer avant qu'elle ne le devienne ! Sans action rapide, l'éradication devient trop chère ou trop difficile. Trouvez un moyen d'inclure des ressources pour lutter contre de nouvelles incursions ainsi que d'autres projets d'éradication réalisables.

- Contrôle biologique (partie du C2 Gestion). D'ordinaire, le contrôle biologique moderne ne présente aucun risque dans la mesure où les directives internationales sont respectées (voir Ressources pp. 42-43) ; toutefois, les craintes qu'il inspire, provoquées par les erreurs du passé (tels la mangouste, le mainate, le crapaud-buffle) commises antérieurement à l'introduction de l'analyse des risques et du test de spécificité, aboutissent souvent à son exclusion des projets. Dans les faits, le contrôle biologique peut traiter, en toute sécurité, de nombreuses espèces envahissantes bien connues dans les îles, notamment des plantes et des insectes envahissants - voir Ressources (pp. 42-43) pour les listes de projets de lutte biologique qui ont abouti. Rappelez-vous que le contrôle biologique peut ne pas coûter si cher si votre cible dispose déjà d'agents de contrôle bien documentés. Esssayez de considérer certaines cibles appropriées, et convainquez les personnes de la valeur de la lutte biologique grâce à la publicité mettant en vedette les succès ainsi que la bonne et rigoureuse démarche scientifique. Et si vous réussissez à mener à bien un tel projet (ce qui signifie non seulement un contrôle réussi, mais aussi sans conséquence néfaste), cela peut changer radicalement l'opinion publique locale.

Des autres domaines négligés qui impliquent tous les dix domaines thématiques de la Checklist de ces Directives, mentionnons :

- Les espèces envahissantes marines et d'eau douce (gardez à l'esprit que ces Directives s'appliquent à tous les types d'espèces envahissantes). Assurez-vous de ne pas oublier ce qui envahit vos eaux côtières et intérieures. Si rien n'a été fait au sujet des espèces aquatiques envahissantes, la première étape consistera à mener des enquêtes de base (B1). Les guides pour la gestion des parasites marins (Hilliard, 2005, Jackson, 2008) peuvent aider à planifier un programme sur les espèces marines envahissantes.

- Les espèces « indigènes envahissantes ”. Considérez ce qu'il faut faire à propos des espèces problématiques qui sont indigènes à votre île (comme beaucoup de mauvaises herbes agricoles ou l'étoile de mer Couronne d'épines pourraient l'être). II arrive que les choses se compliquent quand on ne sait pas vraiment si une espèce est indigène ou pas (comme la vigne Merremia peltata dans le Pacifique). Considérez si de telles espèces problématiques doivent être abordées dans votre plan et si tel est le cas, comment les aborder.

Lorsque vous parcourez la Checklist lors de vos séances de planification, il est important de mettre l'accent sur les domaines ci-dessus qui ont tendance à être oubliés. Déterminez si vous pouvez faire quelque chose à leur sujet et si tel est le cas, comment obtenir des ressources financières pour les inclure. 


\section{Prioriser les espèces, les voies d'introduction et les sites}

Ce type de décision liée à la hiérarchisation des priorités est examiné à la partie B2 de la Checklist de ces Directives. Il existe plusieurs outils d'évaluation des risques (ER), d'analyse des voies d'introduction et d'analyse de l'importance des sites pour vous aider à prendre des décisions sur les espèces, les voies d'introduction et les sites prioritaires à gérer, et un bon aperçu est donné par McGeoch et al. (2016). Pourquoi utiliser ces outils ? - Parce qu'ils vous aident à prévoir les impacts et à utiliser les fonds judicieusement.

\section{Évaluer les risques liés aux ESPÈCES et les prioriser}

L'évaluation des risques essaie de déterminer la probabilité qu'une espèce devienne envahissante dans votre île ou dans vos îles, et peut être utilisée de deux façons : 1) pour évaluer les espèces qui ne sont pas encore présentes sur une île, afin de déterminer si leur importation peut être autorisée ou refusée (contrôle aux frontières ou biosécurité) ; (2) pour évaluer les espèces déjà présentes, p. ex., les plantes en culture, afin de déterminer celles qui sont les plus susceptibles de devenir (plus) envahissantes à l'avenir, et d'aider à les prioriser pour la gestion. Elle peut donc être utilisée pour répondre à plusieurs questions différentes. Une question typique de contrôle aux frontières ou d'un département de quarantaine peut être :

Quelqu'un propose d'introduire une nouvelle plante ornementale ou espèce végétale. L'autorisonsnous à entrer?

Alors que les questions sur la gestion des espèces introduites établies comprennent :

Nous avons 500 espèces végétales introduites dans notre île, et nous connaissons les 10 espèces les plus envahissantes. Mais lesquelles des nombreuses plantes dans les jardins à domicile pourraient se transformer en problèmes prochainement?

Pour les plantes, on parle généralement du système d'« évaluation du risque des adventices » (ERA) et, pour les animaux et les pathogènes, de " l'analyse du risque phytosanitaire » (ARP). Les systèmes d'évaluation des risques vous permettent de prédire le caractère envahissant et les impacts d'une espèce avant qu'ils ne se produisent. Vous pouvez donc décider de refuser la permission d'introduire une espèce dans une île, ou vous pouvez décider d'essayer d'éradiquer une espèce qui est déjà présente, qui n'est pas encore devenue un problème, mais qui le sera probablement à l'avenir si elle n'est pas gérée. Dans les deux cas, vous pouvez agir alors que cela est encore faisable, par conséquent, l'évaluation des risques équivaut à l'utilisation judicieuse des ressources. Le but ultime de l'évaluation des risques est d'aider à prioriser les actions de gestion. L'ER n'établit pas nécessairement les priorités pour vous, mais la sélection de ces dernières a lieu après que les risques ont été évalués.

Quelques principes à respecter au moment de décider contre quoi lutter :

- Toutes les espèces introduites ne sont pas envahissantes, et la priorité doit être donnée à l'action qui traite celles qui causent actuellement, ou qui peuvent causer, le plus de dommages.

- Pour maximiser l'efficacité et l'optimisation des ressources, l'évaluation des risques et la hiérarchisation des espèces envahissantes sont fondamentales et doivent être fondées sur de bonnes connaissances scientifiques.

- Appliquez toujours le « principe de précaution ». Lorsque les connaissances sont insuffisantes pour évaluer avec précision le risque qu'une espèce devienne envahissante, ou ses impacts présents ou futurs, il faut supposer que des impacts se produiront et que des mesures doivent être prises pour empêcher l'espèce de s'établir ou de se propager. Le principe de précaution assume que toute espèce importée dans une île pour être conservée dans des étangs, des enclos, des cages ou des jardins finira par s'enfuir dans la nature, ce qui est généralement le cas! Alors, planifiez en conséquence.

- Lors de la hiérarchisation pour des actions les espèces, les voies et les sites, tenez compte du coût, de l'efficacité, de l'acceptabilité et des problèmes futurs (p. ex., possibilité de réinvasion).

- Une fois que vous les avez choisies, respectez les priorités convenues !

L'ER comprend l'évaluation du risque d'entrée, d'établissement, de dissémination et d'impact d'une espèce, et l'évaluation de l'efficacité et de la faisabilité des options de gestion. Les résultats sont ensuite utilisés pour décider ou recommander des actions (y compris influencer les décideurs). Les évaluations des risques utilisent l'évidence qu'une espèce a été signalée comme envahissante quelque part ou possède des caractéristiques qui mènent habituellement à une invasion, combinées à des connaissances sur l'île concernée par rapport aux endroits où l'espèce est envahissante (climat, sols, zone de végétation, etc.). L'évidence est catégorisée numériquement pour signaler la probabilité que cette espèce devienne envahissante dans l'île concernée. 
Pour le contrôle aux frontières (décisions d'autoriser ou non l'importation), toutes les informations utilisées pour l'ER se basent sur le comportement de l'espèce ailleurs, et le résultat obtenu est exprimé comme recommandation de gestion (c.-à- $d$. " probablement assez sûre pour permettre l'importation ", $\mathrm{OU}$ “ certainement dangereuse, interdire l'importation »). Certains pays utilisent les résultats de l'ER pour générer des listes d'espèces interdites à l'importation (" listes négatives » ou « listes noires »). Une approche plus sûre et plus complète est basée sur des listes d'espèces autorisées à entrer (« listes positives » ou « listes blanches ») avec toutes les autres automatiquement interdites sauf si elles sont testées par l'ER et explicitement autorisées.

Pour les espèces déjà présentes, les informations locales sur les espèces sont également prises en compte dans l'ER, et les scores résultants sont interprétés directement comme des priorités ou classifiés selon la probabilité que les espèces deviennent envahissantes ou pas. Ce type d'ER pour les espèces établies est bien développé pour les plantes (= ERA) et est un excellent moyen de justifier l'éradication précoce des espèces très dangereuses ( $p$. ex. des plantes ornementales) avant qu'elles ne s'enfuient des jardins. Vous trouverez des exemples de systèmes d'ER dans Ressources, p. 42.

\section{Évaluer les risques liés aux VOIES D'INTRODUCTION et les prioriser}

II existe de nombreuses « voies d'introduction » par lesquelles les espèces peuvent être introduites dans les îles, certaines non intentionnelles, d'autres délibérées, et la croissance continue du commerce mondial, des voyages, des transports et du tourisme a créé de nombreuses nouvelles voies d'introduction. Un papillon de nuit nuisible peut être attiré dans un avion éclairé embarquant des passagers ou des marchandises la nuit; des fourmis et des crapauds peuvent ramper dans un conteneur et partir pour d'autres horizons quelques heures plus tard. Les animaux domestiques et le commerce horticole sont deux voies majeures d'introduction délibérée de nouvelles espèces qui deviennent des espèces envahissantes. Les plantes d'aquarium récoltées en Asie peuvent être vendues dans une animalerie de l'île Maurice, puis jetées et emportées dans un drain qui finit dans une zone humide. Une fois ces voies d'introduction identifiées, il est possible de les suivre et de les contrôler. L'évaluation des risques utilisée pour évaluer l'importance des différentes voies d'introduction s'appelle l'Analyse des Voies d'Introduction. En identifiant des voies d'introduction particulières, des biens à risque, etc., cela vous permet de répondre à la question suivante :

\section{Quelles voies d'introduction doivent être gérées de manière prioritaire afin d'empêcher les parasites} connus dans les pays voisins d'entrer dans notre île?

Les multiples voies d'introduction possibles appartiennent aux catégories principales dans le tableau en face. Au sein d'une région insulaire, il est utile d'échanger des informations sur les espèces fréquemment détectées, les arrivées récentes d'espèces potentiellement envahissantes et leurs voies d'introduction communes. Ensuite, vous pouvez suivre ces voies d'introduction pour les espèces attendues et essayer d'empêcher leur entrée ou leur établissement. Les voies d'introduction délibérée sont contrôlées par des approches réglementaires (p. ex., l'octroi de licences) et l'inspection. Les voies d'entrée illégale ou involontaire de nouvelles espèces sont contrôlées par l'inspection et le traitement des navires et des avions, des marchandises, des matériaux de construction, des équipements ( $p$. ex., avec des résidus de sol), des bagages personnels, des colis postaux, etc.

\footnotetext{
Évaluer les risques pour les SITES et les prioriser pour la gestion des espèces envahissantes De nombreux services de conservation établissent des listes de sites prioritaires pour la protection ou pour divers types de gestions, généralement en fonction de la valeur de la biodiversité et des menaces qui pèsent sur elle, y compris les espèces envahissantes. Les organisations locales responsables, bien sûr, donneront la priorité au travail sur les sites qu'elles gèrent. Toutefois, des choix plus difficiles devront peut-être être faits aux échelles nationale et régionale, en particulier en ce qui concerne les îles (ou autres sites majeurs) à gérer lorsque les ressources sont insuffisantes pour tout faire, comme c'est généralement le cas. A l'heure actuelle, les exercices formels de priorisation deviennent donc plus fréquents pour faciliter de telles décisions. Par exemple, la priorité d'une île pour l'éradication des espèces envahissantes peut tenir compte de sa valeur en biodiversité (nombre d'espèces rares ou endémiques) et de la faisabilité de l'éradication des espèces envahissantes ciblées (selon la superficie, la complexité du terrain, la population humaine et le risque de ré-invasion). Près de 2500 îles appartenant aux territoires d'outre-mer du Royaume-Uni ont été évaluées de cette manière par Dawson et al. (2014), qui donne une bonne description des méthodes qui peuvent être utilisées pour prioriser les îles pour la gestion. Des données utiles pour la hiérarchisation des îles se trouvent en ligne dans la Base de données de la biodiversité insulaire menacée (TIB) (Ressources p. 42).
} 
Voies d'introduction (= raisons, dans le cas d'introductions délibérées). (Adaptation de Wittenberg et Cock 2001, Hulme et al. 2008 et IUCN 2017b).

Catégorie

LIBÉRATION :

Introductions intentionnelles

directement dans la nature

\section{Voie}

- Gibier et poisson libérés pour la chasse ou comme source de nourriture

- Libérations pour « enrichir » la flore et la faune indigènes (p. ex., par des sociétés d'acclimatation ou pour l'aménagement paysager)

- Agents de contrôle biologique

- Contrôle de l'érosion, stabilisation des dunes, gestion des déchets, etc.

- Analogues écologiques pour remplacer des espèces ou des populations éteintes

- Introductions d'espèces menacées dans des refuges de conservation

\section{FUITE :}

Introductions intentionnelles sans souci particulier de confinement

Introductions intentionnelles au confinement, qui s'enfuient involontairement

\section{CONTAMINANT :}

Introduction involontaire avec une marchandise
- Plantes (y compris le germoplasme) introduites pour l'agriculture, l'horticulture, les biocarburants, la foresterie ou l'amélioration des sols, y compris par des programmes d'aide au développement

- Plantes ornementales, animaux de compagnie, organismes d'aquarium et leurs aliments vivants, qui s'enfuient ou sont intentionnellement libérés dans la nature

- Animaux d'élevage, y compris les fermes d'élevage d'animaux à fourrure, l'aquaculture et la mariculture

- Fuite de zoos, d'aquariums, de jardins botaniques et d'installations de recherche

- Graines, invertébrés, parasites et agents pathogènes présents dans ou sur les animaux, les plantes et les semences commercialisés pour l'alimentation, l'agriculture, la sylviculture, l'aquaculture, la mariculture et les appâts

- Semences, invertébrés et agents pathogènes des produits agricoles, forestiers, aquacoles et maricoles

- Semences, invertébrés et agents pathogènes qui contaminent des plantes de pépinières, des matériaux et des fleurs coupées

- Espèces vivantes dans le sol et transportées dans le sol
PASSAGER CLANDESTIN :

Associé involontairement à un vecteur de transport
- Organismes sur les machines, le matériel et les véhicules, y compris militaires

- Organismes dans les matériaux d'emballage ou sur ces derniers

- Organismes adhérant aux bagages et à l'équipement des passagers et des touristes, p. ex., équipement de pêche à la ligne

- Auto-stoppeurs dans une cargaison ou sur cette dernière, y compris les conteneurs d'expédition

- Auto-stoppeurs dans des avions ou sur ces derniers

- Organismes présents dans les ballasts secs, les eaux de ballast et les sédiments des citernes de ballasts dans les navires

- Organismes d'encrassement de coque de bateau

- Organismes dans des débris marins synthétiques ou sur ces derniers
CORRIDOR ou FACILITATION: Introduction involontaire, facilitée par l'activité humaine
- Organismes traversant des structures artificielles, comme les poissons dans les canaux, les animaux traversant par les ponts ou les tunnels

- Organismes arrivant sans aide après avoir été introduits à proximité par les voies d'introduction ci-dessus, ou bénéficiant de l'aide des activités humaines, telle la transformation de l'utilisation des terres, pour s'établir 


\section{Comment sélectionner les buts de gestion pour une espèce ou un site}

Une fois que vous avez identifié les espèces, les voies d'introduction et les sites prioritaires sur lesquels vous voulez travailler, comment décidez-vous quoi faire à leur sujet ? L'objectif est-il d'éradiquer complètement l'espèce de l'île ? Est-ce possible (= combien cela pourrait coûter) ? Sinon, l'espèce est-elle une cible potentielle pour le contrôle biologique ? Voulez-vous simplement empêcher les espèces d'entrer dans les zones riches en biodiversité, comme un parc national ? Ou bien si l'espèce est déjà répandue et commune, voulez-vous simplement réduire sa population et ses impacts dans certains endroits comme dans les fermes, proches ou dans les réservoirs d'eau douce ou dans les parcs nationaux ? Ce type de décision correspond au choix du but de gestion, qui est lié au choix de la technique de gestion, mais ces deux décisions sont différentes (p. ex., les techniques de lutte chimique peuvent être utilisées pour l'éradication ou la réduction à long terme de la population - deux buts de gestion très différents).

Pour choisir un but de gestion, une approche hiérarchique devrait être adoptée, dans l'ordre de priorité approximatif comme suit :|||||'||

1. Prévention. La prévention est plus efficace et moins coûteuse que la gestion des espèces envahissantes établies, c'est pourquoi l'exclusion des espèces envahissantes par le contrôle aux frontières est la priorité absolue (renvoi à la Checklist C1, pp. 11-12).

2. Éradication (élimination complète de l'espèce sur une île). Si les impacts de l'espèce dépassent les avantages de l'avoir sur votre île, déterminez si elle peut être complètement éradiquée de l'île. Le succès de l'éradication et le calcul de son coût peuvent être prédits de façon très fiable de nos jours, au

Hiérarchie de buts de gestion

1. Prévention $\| I I \mid>$

2. Éradication IIII $\mid \vec{\gamma}$

3. Réduction permanente de la population ou de sa vigueur $\| I I|\overrightarrow{\mid}\rangle$

4. Confinement IIII $\rangle$

5. La gestion à long terme $\| \mathrm{III}\rangle$

6. Atténuation. moins pour les mammifères et les plantes. Utilisez un modèle formel pour calculer le coût et le temps requis pour l'éradication (p. ex. Cacho et Pheloung 2007 ou IPMDAT). Un programme d'éradication peut être coûteux, mais il s'agit d'un coût ponctuel et, par la suite, le coût de l'espèce nuisible et de sa gestion est presque nul, bien qu'une surveillance continue soit nécessaire pour éviter toute nouvelle invasion. L'éradication est donc plus efficace et moins coûteuse à long terme que le contrôle permanent d'une population de l'espèce ciblée, et l'éradication doit être envisagée chaque fois que possible, en particulier pour les nouveaux arrivants (Checklist objectif $\mathrm{C} 1.4, \mathrm{p} .12$ ) et les espèces présentes seulement dans de zones géographiques restreintes (Checklist action C2.2c, p. 13). L'éradication est plus efficace si une nouvelle arrivée est détectée tôt alors qu'elle est encore limitée en termes d'expansion, ce qui confirme une fois de plus l'importance de la surveillance des espèces envahissantes en contexte insulaire. Assurez-vous que les critères stricts qui doivent être respectés pour une éradication réussie soient prévus par votre projet (IUCN 2000, Parkes et Panetta 2009).

3. Réduction permanente de la population ou de sa vigueur. Les espèces qui ne peuvent pas être éradiquées, en particulier celles qui se sont largement répandues, devraient être considérées comme candidates à la lutte biologique, aux techniques génétiques ou à d'autres moyens similaires de réduction permanente de la population ou de l'impact (Checklist actions C2.2d et C2.2e, p. 13) car ces techniques nécessitent également un investissement à long terme minimal après que le succès a été atteint. Si l'espèce est une plante ou un insecte peu valorisé localement, le contrôle biologique peut être une option. II peut aussi être utilisé pour contrôler certaines plantes envahissantes, tout comme celles qui sont utiles, car cela ne fait que réduire leur compétitivité et leur impact - il ne les élimine pas. La lutte biologique moderne utilise des agents de contrôle très spécifiques (comme les champignons ou les insectes parasites) qui attaquent uniquement les espèces cibles. Les prédateurs généraux comme les platodes, les mainates, les crapauds buffles, les chats et les mangoustes ne devraient jamais être utilisés pour la lutte biologique, car ils ne contrôlent généralement pas l'espèce envahissante ciblée, ayant trop de nourriture de remplacement, et peuvent donc causer l'extinction d'espèces indigènes. Des agents de lutte biologique sûrs sont disponibles pour certains ravageurs et mauvaises herbes à relativement bon marché. Mais si aucun agent connu n'existe pour votre espèce nuisible, un programme de recherche coûteux sera nécessaire pour en trouver un.

4. Confinement. Les espèces qui ne peuvent pas être éradiquées avec les ressources disponibles (utilisez un modèle formel pour vérifier les coûts et le temps requis : Cacho et Pheloung 2007 ou IPMDAT), mais qui ne se sont pas encore largement répandues, ou qui sont utiles ou ont de la valeur pour les personnes et ne peuvent donc pas être éradiquées ni contrôlées biologiquement, devraient être contenues dans des zones délimitées si possible (Checklist action C2.2f, p.13).

5. La gestion à long terme d'une population établie d'un ravageur, y compris l'exclusion des zones prioritaires définies (appelé également contrôle " orienté sur le site »), devrait normalement être 
considérée comme le dernier recours pour la gestion des espèces envahissantes, après que l'éradication, le confinement et la lutte biologique ont tous été jugés infaisables avec les ressources actuelles ou mobilisables (Checklist actions C2.2g et C2.2h, p.13). Dans ce cas, définissez plus précisément le but de gestion. Pourquoi voulez-vous gérer l'espèce (c.-à-d. quels sont les dommages qu'elle cause et où)? Avez-vous besoin de la gérer seulement dans certaines zones ? Une fois ces questions résolues, vous pouvez (1) choisir les meilleures techniques et (2) décider où les utiliser. Des méthodes chimiques, physiques ou génétiques peuvent être utilisées. L'utilisation de ces options implique des coûts permanents et le niveau de contrôle qui peut être atteint dépendra du budget annuel disponible.

6. Atténuation. Lorsque le contrôle de la population d'une espèce envahissante est trop coûteux ou difficile, envisagez l'atténuation de ses impacts par des méthodes autres que la gestion de l'espèce envahissante elle-même (voir Checklist action C2.2i, p.13).

La hiérarchie des buts de gestion ci-dessus, quand les utiliser, et les techniques qui peuvent être utilisées pour les atteindre, peuvent être résumées comme suit :

\begin{tabular}{|c|c|c|c|c|}
\hline Priorité & Objectif de gestion & Techniques utilisées & Caractéristiques des coûts & Plus utile pour \\
\hline 1 & Prévention & $\begin{array}{l}\text { Inspections, piégeage, amorçage, } \\
\text { etc., aux points d'entrée }\end{array}$ & $\begin{array}{l}\text { La méthode la moins chère } \\
\text { pour de mul-tiples espèces }\end{array}$ & $\begin{array}{l}\text { Des espèces pas encore } \\
\text { présentes }\end{array}$ \\
\hline 2 & Éradication & $\begin{array}{l}\text { Physique (tir, piégeage, } \\
\text { déracinement...), chimique } \\
\text { (pesticides et herbicides), génétique } \\
\text { (mâle stérile, transgènes, etc.) }\end{array}$ & $\begin{array}{l}\text { Coûts initiaux élevés, mais } \\
\text { minimes après l'éradication }\end{array}$ & $\begin{array}{l}\text { Des espèces présentes } \\
\text { dans de petites zones, } \\
\text { y compris les nouveaux } \\
\text { arrivants. }\end{array}$ \\
\hline 3 & $\begin{array}{l}\text { Réduction perma- } \\
\text { nente de la taille de } \\
\text { la population, de sa } \\
\text { vigueur ou de son } \\
\text { impact }\end{array}$ & $\begin{array}{l}\text { Contrôle biologique, gestion } \\
\text { génétique des ravageurs }\end{array}$ & $\begin{array}{l}\text { Coûts élevés pour les } \\
\text { nouveaux agents de contrôle, } \\
\text { faibles pour les plus connus, } \\
\text { et minimes après la mise en } \\
\text { place d'un agent de contrôle } \\
\text { efficace. }\end{array}$ & $\begin{array}{l}\text { Espèces répandues, } \\
\text { nuisibles et non utiles }\end{array}$ \\
\hline 4 & Confinement & $\begin{array}{l}\text { Techniques physiques, chimiques } \\
\text { ou génétiques }\end{array}$ & Coûts permanents & $\begin{array}{l}\text { Des espèces utiles mais } \\
\text { nuisibles; des nouvelles } \\
\text { incursions }\end{array}$ \\
\hline 5 & $\begin{array}{l}\text { Gestion de la popu- } \\
\text { lation à long terme, } \\
\text { contrôle « orienté sur } \\
\text { le site », exclusion }\end{array}$ & $\begin{array}{l}\text { Techniques physiques, chimiques } \\
\text { ou génétiques ; barrières multi- } \\
\text { espèces }\end{array}$ & Coûts permanents & $\begin{array}{l}\text { Espèces répandues et } \\
\text { nuisibles pour les-quelles } \\
\text { les objectifs } 1 \text { à } 4 \text { ne sont } \\
\text { pas réali-sables }\end{array}$ \\
\hline 6 & Atténuation & $\begin{array}{l}\text { Protection directe des valeurs ( } \mathrm{p} \text {. } \\
\text { ex., protection des nids, des semis } \\
\text { d'arbres, etc.) }\end{array}$ & Coûts permanents & $\begin{array}{l}\text { Espèces impossibles à } \\
\text { contrôler (objectifs } 1 \text { à } 5 \\
\text { impossibles) }\end{array}$ \\
\hline
\end{tabular}

Accordez plus d'attention aux objectifs 1 à 3 : prévention, éradication, biocontrôle

L'objectif de gestion approprié pour chaque situation dépendra des circonstances individuelles.

Cependant, il est courant que les praticiens entreprennent des programmes de contrôle à long terme (but

5 : gestion de la population à long terme ou contrôle " orienté sur le site »), même si ce n'est généralement pas la meilleure façon d'utiliser un budget limité. La gestion des espèces envahissantes en général doit se détourner du contrôle à long terme et se concentrer sur la prévention, l'éradication et le contrôle biologique, qui ensemble peuvent être beaucoup plus efficaces et permettre de traiter plus de problèmes pour un budget donné. Ces trois options sont négligées en partie parce que l'éradication et la lutte biologique peuvent exiger un investissement initial important ou une expertise spécialisée, tandis que la prévention et l'éradication d'espèces présentes uniquement dans des zones limitées pour l'instant ne semblent pas « agir contre les grands problèmes ». Mais gardez toujours à l'esprit que les buts 5 (contrôle à long terme) et 6 (atténuation des impacts) sont le dernier recours et sont souvent les moyens les moins efficaces de gérer les espèces envahissantes.

\section{Attention au contrôle d'une espèce envahissante en l'utilisant}

L'utilisation d'une espèce envahissante procure des avantages économiques ou autres à quelques personnes, ce qui peut créer un désaccord et ralentir ou même arrêter les efforts de contrôle en créant 
une ressource précieuse (l'espèce envahissante) que ces personnes souhaitent conserver ou même propager. En général, il est déconseillé de baser un programme de contrôle sur la création de revenus en faveur d'une couche de la communauté. Dans les cas où la plupart des parties prenantes estiment qu'une espèce envahissante doit être éradiquée, mais que certaines d'entre elles voient un certain bénéfice à utiliser à long terme l'espèce ciblée,, impliquer la participation de toutes les parties prenantes légitimes aux décisions de gestion peut démontrer qu'il existe en fait un soutien majoritaire pour l'éradication (voir "Clients difficiles » ci-dessus pp. 18-19). Dans de rares cas seulement, il n'y a pas de véritable solution alternative - p. ex., la pêche des savoureuses rascasses envahissantes des récifs des Caraïbes peut aider à gérer cette espèce qui n'est pas sensible aux techniques de gestion conventionnelles. Consultez le tableau ci-dessous pour savoir comment décider de recommander ou non une espèce envahissante à des fins commerciales. NB : La colonne « Ne pas utiliser! " surimpose la colonne « Favorable à l'utilisation »- toute coche dans « Ne pas utiliser! » signifie ne pas utiliser cette espèce du tout.

\section{Comment décider de recommander ou non une espèce envahissante à des fins commerciales}

\section{Facteur}

Espèces non présentes dans nos îles

L'éradication de l'espèce devrait être faisable

L'espèce peut être cultivée/élevée profitablement dans nos îles

La récolte est susceptible d'encourager les bénéficiaires à modifier l'habitat pour permettre à

l'espèce de se propager

Espèce sujette aux parasites ou aux agents pathogènes qui sont envahissants ou possèdent une

large gamme d'hôtes

La commercialisation pourrait attirer des utilisateurs provenant de l'extérieur de nos îles

Espèce faisant facilement l'objet de contrebande

Espèce exclusivement récoltée dans la nature

La récolte est susceptible de réduire la taille de la population ou la distribution de l'espèce I'utilisation

Ne pas utiliser !

$\checkmark v$
$v$
$\checkmark$
$\checkmark$
$\checkmark$
$\checkmark$
$\checkmark$

Adaptation de la St Lucia National Invasive Species Strategy 2012-2021. <www.ciasnet.org/wp-content/uploads/2013/05/NISS-SLU-final-reformat-v6.pdf>

\section{Prendre des décisions difficiles : attitude, pensée positive}

Ne privez pas votre projet prioritaire des ressources nécessaires, surtout s'il s'agit d'une éradication. Allouez-y tout ce dont il a besoin (même un peu plus), pour maximiser vos chances de succès, et si vous avez un surplus dans votre budget, passez à la priorité numéro 2. Ne mettez pas en péril le succès de la priorité numéro 1 en essayant de financer trop de projets et en échouant finalement sur toute la ligne. II vaut mieux entreprendre un projet qui réussit pour ensuite passer au suivant.

Ne laissez pas le manque d'information vous paralyser. Faites quelque chose, apprenez de vos erreurs et adaptez-vous. Si vous vous rendez compte que « personne n'a fait cela auparavant " (technique de gestion) ou si vous ne trouvez aucune information sur la façon de gérer l'espèce $X$, envisagez de faire des essais (puis de suivre et partager les résultats). Chaque première essaie de gestion

Mais, n'essayez pas non plus de "résoudre " un énorme problème avec peu d'argent. Résistez à la pression, laissez le problème tel quel et attaquez-vous à quelque chose qui vous permettra d'apporter votre pierre à l'édifice (gardez à l'esprit le premier point ci-dessus). 


\section{Comment : passer des plans aux actions}

- Un plan ne devrait pas être basé sur « ce que nous aimerions faire » - ce genre de longue liste de souhaits est inutile, car nous savons tous déjà que nous aimerions pouvoir tout accomplir!

- Un bon plan définit « ce que nous allons faire au cours des $X$ prochaines années »

Si votre équipe a suivi ces Directives lors de la rédaction du plan, le plan sera réaliste et réalisable avec les ressources à votre disposition ou facilement mobilisables. Par conséquent, passer du plan aux actions sur le terrain ne devrait pas être trop difficile. C'est tout l'intérêt d'un bon plan - vous devriez être capable d'accomplir les actions qui s'y trouvent. Donc, pour passer de votre bon plan aux actions, tout ce que vous devez faire c'est d'aller de l'avant !

Les actions doivent déjà être clairement identifiées dans votre Plan d'action. Chaque action devient un ou plusieurs projets. La mise en œuvre des projets implique un niveau différent de planification, la «planification opérationnelle », qui fait partie du « cycle de vie du projet ».

\section{Le « cycle de vie du projet»}

\section{Commencer ici}

6. Surtout, n'oubliez pas la durabilité du projet. Suivez les résultats jusqu'à l'aboutissement, diffusez les résultats sur votre réseau régional sur les espèces envahissantes, continuez à surveiller pour détecter toute résurgence ou tout aboutissement indésirable et adaptez votre gestion en réponse aux conclusions.

z'à
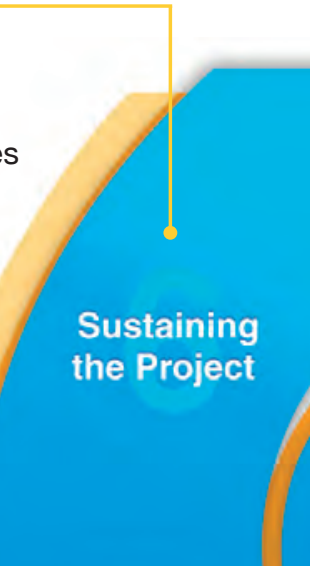
the Project

.

5. Enfin, vous pouvez commencer la mise en œuvre du projet de gestion.

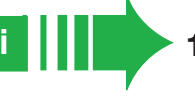

1. Le cycle commence avec la sélection du projet. Cela comprend l'évaluation du risque des espèces envahissantes et des voies d'introduction cibles possibles (voir pp. 31-32), la sélection des cibles prioritaires et la sélection d'un objectif et d'une méthode de gestion pour chacune d'entre elles. Une grande partie de cela aura déjà été couverte au moment d'écrire votre Plan d'action global.

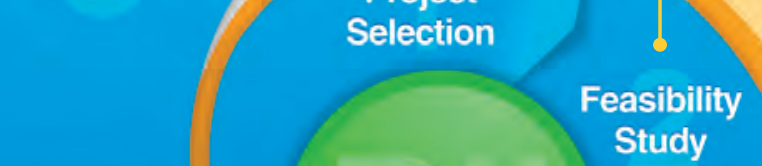

Study

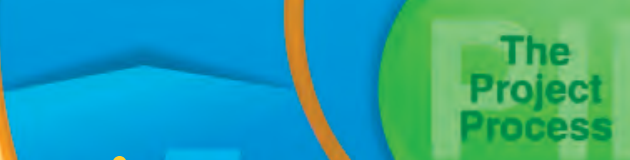

r

\section{Implementation}

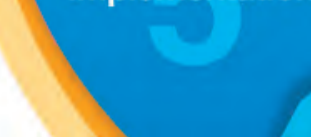

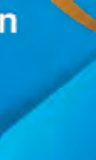

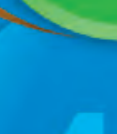
Operational Planning
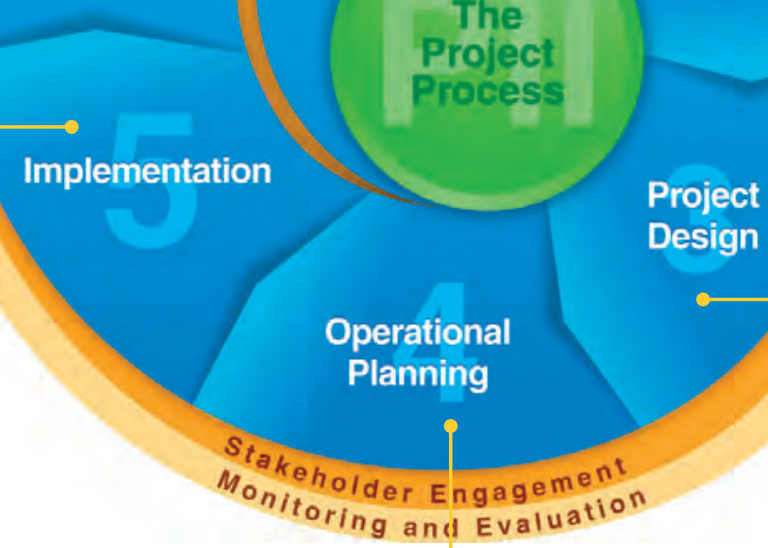

4. Si vous trouvez que vous pouvez toujours vous le permettre, passez à la planification opérationnelle du projet en lui-même.

2. Ensuite, en particulier pour les projets de gestion sur le terrain, il devrait idéalement y avoir une étude de faisabilité pour déterminer si vous pouvez atteindre votre objectif de gestion, et quelles ressources seront nécessaires pour le faire.

\section{Toutes les étapes ci-dessus sont essentielles pour donner au projet les meilleures chances de} succès. 
Tout au long de ce cycle, privilégiez l'inclusion. Comme pour la planification en général, les processus de prise de décision dans la planification de projet doivent impliquer le plus grand nombre de parties prenantes possible. Pour les projets de biosécurité ou d'éradication, il se peut que l'ensemble de la population d'une île ait besoin d'être impliqué. Même lorsqu'il s'agit d'une invasion dans une réserve ou dans une aire protégée (où une organisation de gestion peut être entièrement responsable), rappelezvous que les espèces envahissantes ne connaissent pas les frontières, donc le projet doit englober les zones environnantes pour minimiser la propagation régionale. Si possible, adoptez une approche de planification à l'échelle du paysage et faites participer les propriétaires fonciers environnants.

\section{Après avoir sélectionné votre projet}

Étape 2. L'étude de faisabilité devrait être réalisée par un expert dans ce domaine. Dans la mesure du possible, incluez une analyse coûts-bénéfices, qui non seulement aide à la prise de décision, mais peut également être utilisée pour justifier les demandes de fonds en montrant à la fois le coût de l'inaction et l'avantage économique de financer la gestion proposée. L'analyse coûts-bénéfices devrait également examiner si l'espèce envahissante profite à certaines parties prenantes. Des techniques d'analyse coûtsbénéfices sont décrites dans Emerton et Howard (2008), et des exemples de cette approche dans les îles peuvent être consultés dans Buncle et al. (2013) et Daigneault et Brown (2013).

Étape 3. À l'étape de la conception du projet, le projet devrait être budgétisé en détail afin de confirmer que les fonds et les autres ressources disponibles permettent de maintenir les activités jusqu'à ce que le résultat souhaité soit atteint. Ceci est crucial - de nombreuses actions de gestion sont entreprises avec pas suffisamment de fonds ou de temps à disposition, ce qui entraîne l'échec d'une gestion efficace, ou la résurgence ultérieure de l'envahisseur : $c$.-à- $d$. que l'argent a été gaspillé. La réalisation d'une étude pilote à petite échelle sur un ou plusieurs sites et la saisie des informations détaillées en termes de temps et de coûts du travail peuvent être utilisées pour budgétiser un projet plus conséquent. Incluez le temps et les coûts impliqués pour la collecte des outils et des produits nécessaires, la mobilisation des équipes et de leurs équipements, le travail sur le terrain, le nettoyage, la restauration du site ainsi que l'analyse et la rédaction des résultats. L'estimation de la valeur des contributions en nature est utile pour mobiliser des fonds réels (p. ex., le personnel de l'université $X$ nous donne l'équivalent de 10000 dollars de son temps pour identifier des échantillons, veuillez nous donner les 1000 dollars de salaire dont nous avons besoin pour les prélever).

Étapes 4 et 5. Comme nous l'avons expliqué en introduction, ces Directives n'expliquent pas en détail la planification ni la mise en œuvre du projet, car de nombreuses autres ressources traitent déjà de tout cela, allant de la prévention à la gestion, et pour toute sorte d'animal ou de plante envahissant. Vous pouvez trouver plus de détails sur les meilleures pratiques adaptées aux différents aspects de la gestion dans Ressources pp. 42-43, mais ne vous arrêtez pas là - utilisez vos réseaux sur les espèces envahissantes, comme aliens-I, pour poser des questions et chercher de l'expertise. Pour l'éradication, les groupes consultatifs comprennent Island Conservation (www.isa-conservation.org) et I'Island Eradication Advisory Group du Département de la conservation (DOC) de la Nouvelle-Zélande (www.doc.govt.nz/footer-links/contact-us/).

Étape 6. La durabilité du projet. Si vous avez suivi ces Directives, l'action de gestion que vous avez choisie aura un résultat attendu mesurable. Assurez-vous de le mesurer, $c$.-à- $d$. de collecter et d'analyser efficacement les données de suivi. Le suivi devrait avoir quatre buts : (i) mesurer le succès de l'action de gestion, p. ex., en empêchant l'établissement, ou en réduisant la population de l'espèce envahissante cible, (ii) mesurer le rétablissement de l'écosystème endommagé ou des espèces menacées, ou toute autre valeur endommagée, (iii) vous permettre de détecter tout effet indésirable de la gestion, tel que la mort d'espèces non-ciblées, ou l'invasion par une autre espèce envahissante, (iv) déclencher une action supplémentaire en fonction des résultats mesurés. Le point (iv) est important - effectuer un suivi sans réagir se résume à de la pure observation. Incluez des mesures pour traiter les effets indésirables s'ils se produisent, et incorporez des plans pour la gestion complémentaire des espèces envahissantes ainsi que la restauration de l'écosystème ou d'espèces rares particulières qui pourraient avoir besoin d'une aide supplémentaire. Cela se traduit en une approche écosystémique, avec une gestion adaptative pour vous permettre de gérer des résultats inattendus. Le suivi produit également des informations utiles pour la planification de projets ultérieurs et renvoie donc à l'étape $\mathbf{1}$ du cycle de vie du projet (ce qui explique pourquoi cela s'appelle un cycle). 


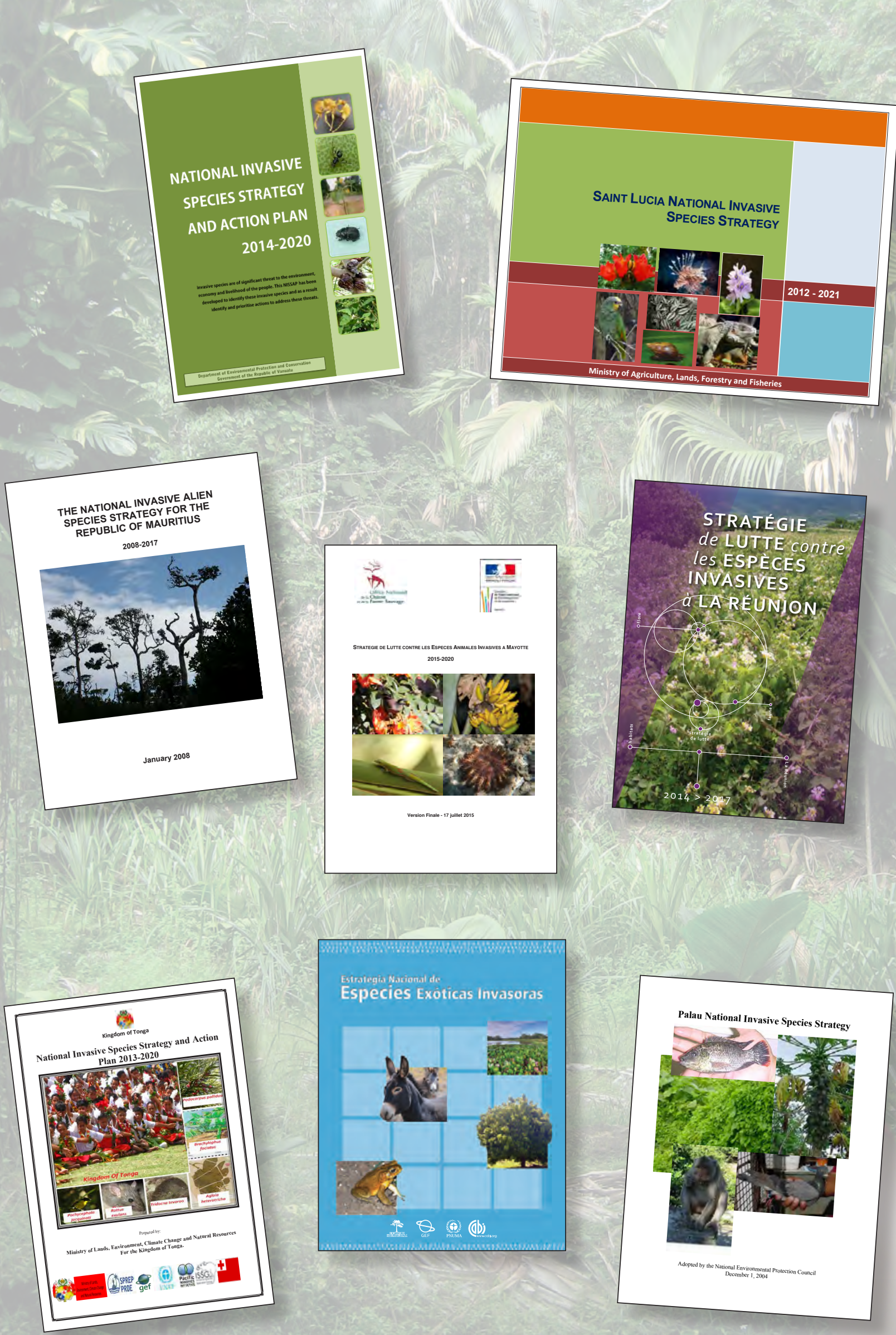

용 


\section{Ressources}

\section{Instruments mondiaux couvrant des questions liés aux espèces envahissantes}

Convention sur la diversité biologique (CDB ou Convention de Rio, article $8 \mathrm{~h}$ exhortant les parties contractantes à gérer les espèces envahissantes) <www.cbd.int/>, et ses Objectifs d'Aichi pour la conservation de la biodiversité (en particulier l'Objectif 9 Compréhension, prévention et gestion des espèces envahissantes) <www.cbd.int/sp/targets/>.

Convention internationale pour le contrôle et la gestion des eaux de ballast et des sédiments des navires (BWMC) <WwW. imo.org/en/About/Conventions/ListOfConventions/Pages/International-Convention-for-the-Control-and-Management-ofShips\%27-Ballast-Water-and-Sediments-(BWM).aspx>

Convention internationale pour la protection des végétaux $<$ www.ippc.int/>

Objectifs de développement durable des Nations Unies <www.un.org/sustainabledevelopment/sustainable-development-goals/>

Organisation mondiale de la santé animale (OIE) <www.oie.int/ $>$

\section{Stratégies et instruments régionaux}

Règles et directives de l'Union européenne sur la prévention et la gestion des espèces exotiques envahissantes <http:// ec.europa.eu/environment/nature/invasivealien/index_fr.htm>. De bons exemples pour l'élaboration de lois et de règlements, les évaluations des risques et d'autres études.

Stratégie et plan d'action sur les espèces envahissantes de l'Atlantique Sud <http://ww2.rspb.org.uk/Images/SAIS_Strategy_ Plan_tcm9-273308.pdf>

\section{Plans nationaux}

Les Stratégies et plans d'action nationaux pour la biodiversité (SPANB) préparés par les parties contractantes de la CDB sont publics et disponibles à <https://www.cbd.int/nbsap/>.

Stratégies nationales de développement <www.un.org/en/development/desa/policynotes.html>

Stratégies et plans d'action nationaux sur les espèces envahissantes (SPANEE). Un grand nombre peut être trouvé en ligne.

\section{Ressources sur plusieurs thèmes de ces directives}

Programme mondial sur les espèces envahissantes

Boîtes à outils et directives : <www.issg.org/gisp_guidelines_toolkits.htm>

Matériel de cours de formation : <www.issg.org/gisp_training_coursematerials.htm>

Battler Resource Base du PILN <https://www.sprep.org/piln/resource-base> : guides pratiques « Comment » sur de nombreux thèmes de ces Directives, y compris les campagnes de sensibilisation, l'analyse économique, les invasions marines, l'utilisation de bases de données en ligne et bien d'autres.

\section{Ressources sélectionnées liées aux parties de la Checklist de ces Directives}

Inclut seulement les références mentionnées dans le texte ou des exemples particulièrement utiles. Cette liste n'est pas exhaustive et beaucoup plus d'informations peuvent être trouvées en ligne ou en effectuant la demande à travers les listes de distribution d'emails sur les espèces envahissantes énumérées ci-dessous.

\section{A1 Analyses des lacunes}

Boudjelas, S. (2018). A review of national and island plans for the management of invasive species in the Western Indian Ocean region. UICN.

Doherty, N. et Boudjelas, S. (2010). Invasive Species Management in the Pacific: a review of national plans and current activities. Rapport non publié pour le Pacific Invasives Partnership. Auckland : Initiative du Pacifique sur les espèces envahissantes. $<$ www.issg.org/cii/Electronic\%20references/pii/references/pii_ism_in_the_pacific_a_review_of_national_plans_and_current_ activities.pdf>. 
Key, J. (2017) Tackling Invasive Non-Native Species in the UK Overseas Territories - Gap Analysis of Biosecurity Capacity. GB Non-native Species Secretariat, York. <https://secure.fera.defra.gov.uk/nonnativespecies/downloadDocument.cfm?id=1566> PROE (2010) Directives relatives à la gestion des espèces envahissantes dans le Pacifique. PROE, Apia.

\section{A2 Obtenir du soutien ; messages de sensibilisation, y compris l'analyse coûts-avantages}

Buncle, A., Daigneault, A., Holland, P., Fink, A., Hook, S. et Manley, M. (2013) Cost-benefit Analysis for Natural Resource Management in the Pacific. A Guide. PROE, Apia. <https://pdfs.semanticscholar.org/5a08/3078ee7f17cebbb9b6dd6b27 fe9b7d782bb4.pdf>

Daigneault et Brown, P. (2013) Invasive species management in the Pacific using survey data and benefit-cost analysis. <https:// ageconsearch.umn.edu/bitstream/152140/2/CP\%20Brown.pdf>

Emerton, L. \& Howard, G., 2008. A toolkit for the economic analysis of invasive species. Programme mondial sur les espèces envahissantes, 110 p. <https://portals.iucn.org/library/node/9248>

Madden, F. \& McQuinn, B. (2014) «Conservation's blind spot : the case for conflict transformation in wildlife conservation. » Biological Conservation 178: 97-106.

Novoa, A. et al. (2018) «A framework for engaging stakeholders on the management of alien species. » Journal of Environmental Management 205: 286-297.

\section{A3 Réseaux et listes de diffusions sur les espèces envahissantes}

Réseau des espèces exotiques envahissantes des Caraïbes (CIASNET) <www.ciasnet.org/>. Liste de diffusion : carib_ias_threat Réseau de conservation des îles <http://listserv.bgci.org/scripts/wa.exe?A0=islands-l>. Liste de diffusion : islands-I

Groupe de spécialistes de I'UICN sur les invasions biologiques (ISSG). <www.issg.org/>. Liste de diffusion : aliens-I

Initiative du Pacifique sur les espèces envahissantes (PII) <www.pacificinvasivesinitiative.org/>. Liste de diffusion pii@auckland. ac.nz

Réseau d'apprentissage sur les espèces envahissantes du Pacifique (PILN) <www.sprep.org/piln>.

Partenariat océanien sur les espèces envahissantes (PIP) <www.sprep.org/Pacific-Invasives-Partnership/invasive-partnerships $>$

Pestnet <www.pestnet.org/PestNet.aspx $>$. Liste de diffusion : pestnet.

Réseau de l'océan Indien occidental sur les espèces envahissantes (WIONIS). <www.agriculture-biodiversite-oi.org/en/WIONIS/ WIONIS-the-Network wio-ias>. Liste de diffusion : wio-ias.

\section{A3 Organisations et initiatives régionales}

Communauté des Caraïbes (CARICOM) <http://caricom.org/about-caricom/who-we-are>

Centre d'activités régional pour le protocole relatif aux zones et à la vie sauvage spécialement protégées de la Grande Région Caraïbe (CAR-SPAW-RAC) <www.car-spaw-rac.org/>

Commission de l'océan Indien (COI) <www.commissionoceanindien.org/>

Programme régional océanien de l'environnement (PROE) <www.sprep.org $>$

Comité français de I'UICN Initiative sur les Espèces Exotiques Envahissantes en Outre-mer. <www.especes-envahissantesoutremer.fr/>

La Communauté du Pacifique (CPS) <www.spc.int $>$

\section{A3 Ressources informatives en ligne sur les espèces envahissantes}

Compendium sur les espèces envahissantes $\mathrm{CABI}<$ www.cabi.org/isc $>$ : informations détaillées sur les parasites, les mauvaises herbes et les maladies, la taxonomie, le caractère envahissant, la distribution, la gestion.

DAISIE <www.europe-aliens.org/aboutDAISIE.do> : informations sur les espèces envahissantes en Europe, y compris de nombreuses îles.

Base de données sur l'éradication des espèces envahissantes insulaires (DIISE) <http://diise.islandconservation.org/>

FishBase <www.fishbase.org/> : permet d'effectuer des recherches par pays pour les poissons introduits ou envahissants.

Système mondial d'information sur la biodiversité (GBIF) <www.gbif.org/> : dossiers et taxonomie.

Base de données mondiale sur les espèces envahissantes (GISD) <www.iucngisd.org/gisd/> : informations détaillées sur certaines espèces envahissantes.

Registre mondial des espèces introduites et envahissantes (GRIIS) <www.griis.org/> : listes des espèces par pays.

HEAR <www.hear.org> : de nombreux sujets relatifs aux envahisseurs, y compris le Compendium mondial des mauvaises herbes $<$ www.hear.org/gcw/ $>$ (> 20000 taxons avec des références bibliographiques).

Le Réseau d'information sur les espèces envahissantes de l'IABIN (I3N) <www.institutohorus.org.br/iabin/i3n/> : informations sur les espèces envahissantes dans les Amériques, y compris de nombreuses îles.

Base de données sur les espèces envahissantes et la biodiversité insulaire (IBIS) <http://ibis.fos.auckland.ac.nz/> : quelles espèces envahissantes menacent les espèces endémiques?

Pacific Islands Ecosystems at Risk (PIER) <www.hear.org/pier/>: listes et informations sur les plantes envahissantes dans les îles du Pacifique et ailleurs. Les évaluations préeffectuées des risques de mauvaises herbes sont disponibles sur <www. hear.org/pier/wralist.htm>. 
Base de données sur les organismes nuisibles dans le Pacifique <www.spc.int/pld/> : registres relatifs aux organismes nuisibles dans les îles du Pacifique.

PI@ntNet <https://plantnet.org/> : faites identifier vos photos de plantes !

Base de données sur la biodiversité insulaire menacée (TIB) <http://tib.islandconservation.org/> : informations utiles pour hiérarchiser les îles pour la gestion.

Conseil national des espèces envahissantes (NISC) des États-Unis <www.invasivespeciesinfo.gov/resources/databases.shtml> : informations sur de nombreuses espèces envahissantes communes.

WikWIO <http://www.wikwio.org/idao/> : outil d'identification des mauvaises herbes.

Mauvaises herbes d'importance nationale (WONS : Australien) <www.weeds.org.au/WONS/> : comptes rendus de certaines des pires plantes envahissantes plus techniques de gestion.

Registre mondial des espèces marines introduites (WRIMS) <www.marinespecies.org/introduced/>

\section{A4 Législation, politique et réglementation}

Des modèles de politiques et de législation pour la gestion des espèces envahissantes sont disponibles auprès du Centre juridique de I'UICN (<www.iucn.org/about/work/programmes/environmental_law $>$ ), lequel peut donner des conseils sur différents types de législation pour différentes situations, y compris les îles et les nations insulaires.

Shine, C. (2008). A Toolkit for Developing Legal and Institutional Frameworks for Invasive Alien Species. Programme mondial sur les espèces envahissantes. <www.issg.org/gisp_guidelines_toolkits.htm>

\section{B2 Priorisation et analyse des risques}

Dawson, J., Oppel, S., Cuthbert, R.J., Holmes, N., Bird, J.P., Butchart, S.H.M., Spatz, D.R. et Tershy, B. (2014) « Prioritizing islands for the eradication of invasive vertebrates in the United Kingdom overseas territories. "Conservation Biology 29: 143-153.

Hulme, P.E., Bacher, S., Kenis, M., Klotz, S., Kühn, I., Minchin, D., Nentwig, W., Olenin, S., Panov, V., Pergl, J., Pysek, P., Roques, A., Sol, D., Solarz, W. \& Vilà, M. (2008) « Grasping at the routes of biological invasions: a framework for integrating pathways into policy. » Journal of Applied Ecology 45: 403-414.

IUCN (2017b) Guidance for interpretation of CBD categories on introduction pathways. Note technique préparée par I'UICN pour la Commission européenne. <https://circabc.europa.eu/sd/a/738e82a8-f0a6-47c6-8f3b-aeddb535b83b/TSSR-2016010\%20CBD\%20categories\%20on\%20pathways\%20Final.pdf>

McGeoch, M.A., Genovesi. P., Bellingham, P.J., Costello, M.J., McGrannachan, C. \& Sheppard, A. (2016) « Prioritizing species, pathways, and sites to achieve conservation targets for biological invasion. 》 Biological Invasions 18: $299-314$.

Évaluation des risques des parasites (PRA) et des mauvaises herbes (WRA), systèmes et exemples largement utilisés : WRA Australie <www.agriculture.gov.au/biosecurity/risk-analysis/weeds/system>

WRA Hawai'i <www.botany.hawaii.edu/faculty/daehler/wra/>

Formation GIEC sur le PRA <https://www.ippc.int/en/core-activities/capacity-development/training-material-pest-riskanalysis-based-ippc-standards/>

Beaucoup d'autres exemples peuvent être trouvés en cherchant sur Internet.

\section{C1 et C2 Prévention et gestion des meilleures pratiques}

Meilleure pratique générale

IUCN (2000) Guidelines for the Prevention of Biodiversity Loss Caused by Alien Invasive Species. UICN, Gland. https://portals. iucn.org/library/node/12413

IUCN (2017a) Guidance Note for Pest Management Planning. Système de gestion environnementale et sociale, UICN, Gland.

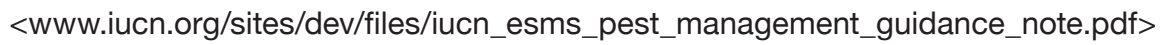

Rocamora, G. \& Henriette, E. (2015) Invasive Alien Species in Seychelles. Why and how to eliminate them. Biotope, Mèze. (Guide de bonnes pratiques, utile pour les îles partout.)

Wittenberg, R. et Cock, M.J.W. (eds.) (2001) Invasive Alien Species: A Toolkit for Best Prevention and Management Practices. Programme mondial sur les espèces envahissantes. <www.issg.org/gisp_guidelines_toolkits.htm>

\section{Biosécurité}

Les codes de conduite de la Convention de Berne pour la gestion des voies d'introduction. Disponible sur : <https://www.coe. int/en/web/bern-convention/on-invasive-alien-species\#\{"12475592":[1]\}>

Conseil sur les espèces envahissantes (2017) Environmental Biosecurity: Best Practice. <https://invasives.org.au/publications/ biosecurity-best-practice/>

Contrôle biologique

CRC Weed Management (2008) Guides des pratiques exemplaires :

1 Impact evaluation of weed biological control agents. <www.utas.edu.au/__data/assets/pdf_file/0011/636149/Best-

Practice-Guide-1-Impact-evaluation-of-weed-biological-control-agents.pdf>,

2 Release and establishment of weed biological control agents. <www.utas.edu.au/_data/assets/pdf_file/0004/373567/

Best-Practice-Guide-2-Release-and-establishment-of-weed-biological-control-agents.pdf >

Day, M.D. et Winston, R.L. (2016) « Biological control of weeds in the 22 Pacific island countries and territories: current status and future prospects. " Neobiota 30: 167-192. 
iBiocontrol. Biological Control of Weeds: a world catalogue of agents and their target weeds. <www.ibiocontrol.org/catalog/> Outils et principes d'éradication

Cacho, O. et Pheloung, P. (2007) WeedSearch weed eradication feasibility analysis. Université de Nouvelle-Angleterre, Armidale. Manuel et logiciel disponible sur :

<https://www.une.edu.au/staff-profiles/business/ocacho> Outil d'évaluation des coûts et de la faisabilité de l'éradication.

IPMDAT. Invasive Plant Management Decision Analysis Tool. <www.ipmdat.org/home.html> Outil d'évaluation des coûts et de la faisabilité de l'éradication.

Parkes, J.P. et Panetta, F.D. (2009) « Eradication of invasive species: progress and emerging issues in the $21^{\text {st }}$ century. » Pp. 47-60 en Clout, M.N. et Williams, P.A. (eds) Invasive Species Management. A handbook of principles and techniques. Oxford University Press, Oxford. Critères d'éradication

\section{Techniques génétiques}

Harvey-Samuel, T., Ant, T. et Alphey, L. (2017) « Towards the genetic control of invasive species. 》 Biological Invasions 19: 1683-1703. Examen du potentiel d'utilisation pour l'éradication, la réduction de la population, le confinement, etc.

\section{Gérer des types particuliers d'organismes}

Orueta, J. (2003) Manual práctico para el manejo de vertebrados invasores en islas de España y Portugal. <www.interregbionatura.com/especies/docs/ManualPracticoVertebradoslslas.pdf>

Thomas, S., Varnham, K. \& Havery, S. (2017) Current Recommended Procedures for UK (Bait Station) Rodent Eradication Projects. Version 4.0, Royal Society for the Protection of Birds. Disponible sur : <www.nonnativespecies.org/index.cfm?pageid=613> New Zealand MFAT (2016) Pacific Invasive Ant Toolkit. <www.piat.org.nz/>

Pacific Invasives Initiative (2011) Resource Kit for Rodent and Cat Eradication. <http://rce.pacificinvasivesinitiative.org/>

Pacific Invasives Initiative (2015) Resource Kit for Invasive Plant Management. <http://pacificinvasivesinitiative.org/ipm/ipm. pacificinvasivesinitiative.org/index.html>

\section{Espèces envahissantes marines}

Programme Globallast : <http://archive.iwlearn.net/globallast.imo.org/> Ressources sur la gestion des eaux de ballast et des sédiments des navires.

Hilliard, R. (2005) Best Practice for the Management of Introduced Marine Pests-A Review. Programme mondial sur les espèces envahissantes. <www.issg.org/gisp_guidelines_toolkits.htm>

Jackson, L. (2008) Guidelines for the Prevention and Management of Marine Biofouling and Invasive Species. Programme mondial sur les espèces envahissantes. <www.issg.org/gisp_guidelines_toolkits.htm>

\section{C3 Restauration}

Société pour la restauration écologique <www.ser.org> : beaucoup d'informations sur une variété d'approches. 




\section{UICN}

UNION INTERNATIONALE POUR LA

CONSERVATION DE LA NATURE

SIĖGE SOCIAL MONDIAL

Rue Mauverney 28

1196 Gland, Suisse

Tél: +41 229990000

Fax: +41 229990002

www.iucn.org 